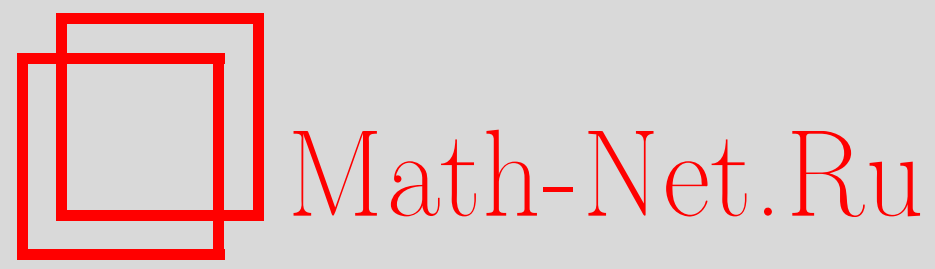

А. А. Гончар, С. П. Суетин, Об аппроксимациях Паде мероморфных функций марковского типа, Совр. пробл. матем., 2004, выпуск 5, 3-67

DOI: https://doi.org/10.4213/spm8

Использование Общероссийского математического портала MathNet.Ru подразумевает, что вы прочитали и согласны с пользовательским соглашением

http://www . mathnet.ru/rus/agreement

Параметры загрузки:

IP: 3.85 .5 .30

26 апреля 2023 г., 13:46:03 


\title{
Об аппроксимациях Паде мероморфных функций марковского типа
}

\author{
А. А. Гончар, С. П. Суетин
}

\section{$\S 1$. Введение}

1. Теория аппроксимаций Паде - локально наилучших рациональных аппроксимаций степенного ряда - составляет важное направление в комплексном анализе и теории приближений. B XVIII-XIX вв. это направление развивалось, в основном, в рамках классической теории непрерывных дробей. Интерес к аппроксимациям Паде и более общим конструкциям рациональных аппроксимаций аналитических функций резко возрос во второй половине прошлого века. Благодаря современному развитию вычислительной техники такие аппроксимации нашли многочисленные применения в различных задачах математической физики, механики и прикладной математики. В свою очередь, анализ возникающих при этом математических проблем привел к новым задачам и результатам в комплексном анализе, теории потенциала, теории ортогональных многочленов (см. [1], где имеется обширная библиография работ в рассматриваемом направлении).

Подчеркнем, что речь идет о приближениях рациональными функциями со свободными полюсами, а сами рассматриваемые аппроксимации - непрерывные дроби, аппроксимации Паде и их различные обобщения - имеют нелинейный характер. Оптимальный выбор коэффициентов как числителей, так и знаменателей аппроксимаций Паде позволяет с их помощью локализовать особенности функции, заданной своим разложением в степенной ряд, и осуществлять ее эффективное аналитическое продолжение за пределы круга сходимости степенного ряда. В этом состоит принципиальное преимущество рассматриваемых рациональных

(C) Гончар А. А., Суетин С. П., 2004 
аппроксимаций перед полиномиальными приближениями и рациональными аппроксимациями с заранее фиксированными полюсами.

Настоящая работа посвящена асимптотическим свойствам диагональных аппроксимаций Паде для мероморфных функций марковского типа. Многие замечательные свойства аппроксимаций Паде и их обобщений были открыты сначала эмпирически в результате численных расчетов и лишь затем строго обоснованы. Именно так была сделана и эта работа: сначала с помощью компьютерных расчетов было обнаружено новое свойство, связанное с асимптотическим поведением полюсов диагональных аппроксимаций Паде, а затем, с целью доказательства того, что соответствующая закономерность имеет место в достаточно широком классе мероморфных функций, были получены новые формулы сильной асимптотики для диагональных аппроксимаций Паде и их знаменателей.

2. Пусть

$$
f=\sum_{k=0}^{\infty} \frac{c_{k}}{z^{k+1}}
$$

- степенной ряд с центром в бесконечно удаленной точке $z=\infty$, $f_{n}=[n / n]_{f}, n=1,2, \ldots,-$ последовательность его диагональных ${ }^{1}$ аппроксимаций Паде. В случае, когда все ганкелевы определители

$$
H_{n}=\left|\begin{array}{cccc}
c_{0} & c_{1} & \ldots & c_{n} \\
c_{1} & c_{2} & \ldots & c_{n+1} \\
\ldots \ldots \ldots \ldots \ldots \ldots & \ldots \ldots \\
c_{n} & c_{n+1} & \ldots & c_{2 n}
\end{array}\right|, \quad n=0,1, \ldots
$$

отличны от нуля, ряду (1) с помощью классического алгоритма Евклида сопоставляется чебышевская непрерывная дробь [2]:

$$
f \sim \frac{a_{1}}{z-b_{1}-\frac{a_{2}}{z-b_{2}-\cdot}}
$$

\footnotetext{
${ }^{1}$ Термин "диагональная последовательность" связан с тем, что такие аппроксимации составляют главную диагональ в общей таблище Паде ряда $f$ (см. [1]).
} 
$n$-я подходящая дробь которой совпадает с $f_{n}$ и обладает следующим характеристическим свойством:

$$
\left(f-f_{n}\right)(z)=\frac{A_{n}}{z^{2 n+1}}+\cdots,
$$

где $A_{n} \neq 0$. Тем самым, дробь (2) есть просто другая (эквивалентная) форма записи для последовательности $\left\{f_{n}\right\}_{n \in \mathbb{N}}$.

В общем случае удобно основываться на следующем определении $n$-й диагональной аппроксимации Паде ряда $f$ (определение Фробениуса): $f_{n}=P_{n} / Q_{n}$, где $P_{n}, Q_{n}$ - полиномы от $z$, $\operatorname{deg} P_{n}<\operatorname{deg} Q_{n} \leqslant n \quad\left(Q_{n} \not \equiv 0\right)$ и выполняется соотношение

$$
R_{n}(z):=\left(Q_{n} f-P_{n}\right)(z)=O\left(\frac{1}{z^{n+1}}\right), \quad z \rightarrow \infty .
$$

Этими условиями полиномы $P_{n}$ и $Q_{n}$ определяются не однозначно, но рациональная дробь $f_{n}=P_{n} / Q_{n}$ единственна (с точностью до стандартного отождествления). Функция $R_{n}$ называется бункцией остатка. Для нормальных индексов $n$ порядок рациональной функции $f_{n}$ равен $n$. Тем самым, для таких $n$ полиномы $P_{n}$ и $Q_{n}$, удовлетворяющие условию $(4)$, не имеют общих нулей, $\operatorname{deg} Q_{n}=n$ и выполняется соотношение (3) (где, возможно, $\left.A_{n}=0\right)$. Каково бы ни было $n$, справедливо равенство $f_{n}=f_{n_{k}}$, где $n_{k}-$ наибольший нормальный индекс, не превосходящий $n$. Тем самым, последовательность нормальных индексов $\left\{n_{k}\right\}=\Lambda=\Lambda(f)$ для ряда $f$ разбивает натуральный ряд чисел на множества $\Lambda_{k}=\left\{n: n_{k} \leqslant n<n_{k+1}\right\}$ такие, что $f_{n}=f_{n_{k}}$ для $n \in \Lambda_{k}$. Такая структура последовательности $\left\{f_{n}\right\}_{n \in \mathbb{N}}$ позволяет в вопросах сходимости диагональных аппроксимаций Паде ограничиться рассмотрением последовательности $\left\{f_{n}\right\}_{n \in \Lambda}$.

Для марковской функции

$$
\widehat{\sigma}(z):=\int \frac{d \sigma(x)}{z-x}, \quad z \in \widehat{\mathbb{C}} \backslash S,
$$

где $\sigma$ - положительная борелевская мера с бесконечным носителем $S=\operatorname{Supp} \sigma \Subset \mathbb{R}$, коэффициенты соответствующего ей ряда (1) совпадают с моментами меры $\sigma$ :

$$
c_{n}=\int x^{n} d \sigma(x), \quad n=0,1,2, \ldots
$$


При этом все определители $H_{n}$ положительны, полиномы $Q_{n}(z)=$ $Q_{n}(z ; \widehat{\sigma})$ имеют степень $n$ и при нормировке $Q_{n}(z ; \widehat{\sigma})=z^{n}+\cdots$ определяются единственным образом:

$$
Q_{n}(z ; \widehat{\sigma})=\frac{1}{H_{n-1}}\left|\begin{array}{cccc}
c_{0} & c_{1} & \ldots & c_{n} \\
c_{1} & c_{2} & \ldots & c_{n+1} \\
\ldots \ldots & \ldots & \ldots & \ldots \ldots \\
c_{n-1} & c_{n} & \ldots & c_{2 n-1} \\
1 & z & \ldots & z^{n}
\end{array}\right|=z^{n}+\cdots, \quad n=1, \ldots
$$

Полиномы $Q_{n}$ ортогональны по мере $\sigma$, при этом для функции остатка (4) справедливо представление

$$
R_{n}(z)=\int \frac{Q_{n}(x) d \sigma(x)}{z-x}, \quad z \in \widehat{\mathbb{C}} \backslash S,
$$

а для полиномов $P_{n}-$ представление

$$
P_{n}(z)=\int \frac{Q_{n}(z)-Q_{n}(x)}{z-x} d \sigma(x) ;
$$

в теории ортогональных многочленов (6) принято называть функцией второго рода, а (7) - многочленами второго рода.

Классическая теорема Маркова [3] утверждает, что для мар-

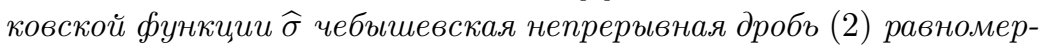
но сходится внутри (на компактных подмножествах) области $D=\widehat{\mathbb{C}} \backslash[\alpha, \beta]$, где $[\alpha, \beta]-$ выпуклая оболочка $S$.

3. В 1975 году в работе [4] одним из авторов настоящей статьи было получено обобщение теоремы Маркова на класс мероморфнъх функций вида

$$
f=\widehat{\sigma}+r
$$

где $r$ - рациональная функция с комплексными коэффициентами $(r$ - "рациональное возмущение" марковской функции $\widehat{\sigma})$. Основной результат работы [4] состоит в следующем ${ }^{2}$.

ТЕОРЕма 1. Пусть $\sigma$ - положительная борелевская мера $c$ носителем Supp $\sigma=[\alpha, \beta], \sigma^{\prime}>0$ n.в. на $[\alpha, \beta]$ u $f=\widehat{\sigma}+r$, где

\footnotetext{
${ }^{2} \mathrm{~B}[4]$ мера $\sigma$ предполагалась такой, что имеет место асимптотика отношения многочленов, ортогональных на $[\alpha, \beta]$ относительно $\sigma$. Позднее Е. А. Рахмановым [5], [6] было показано, что при условии: $\sigma^{\prime}>0$ п.в. на $[\alpha, \beta]$, эта асимптотика отношения имеет место.
} 
$r$ - произвольная комплексная рациональная функция, все полюсъ которой принадлежат области $\mathbb{C} \backslash[\alpha, \beta]$, u $r(\infty)=0$. Тогда последовательность диагональных аппроксимаций Паде $\left\{f_{n}\right\}_{n \in \mathbb{N}}$ сходится $\kappa f$ равномерно в сферической метрике ${ }^{3}$ внутри облаcmu $D=\widehat{\mathbb{C}} \backslash[\alpha, \beta]$.

Из теоремы 1 вытекает, что к каждому полюсу функции $f$ в $D$ стремится (со скоростью геометрической прогрессии; см. ниже формулу (10)) ровно столько полюсов $f_{n}$, какова его кратность, а все предельные точки остальных полюсов лежат на отрезке $[\alpha, \beta]$. Отметим, что из указанного асимптотического поведения полюсов диагональных аппроксимаций Паде для формального ряда $f$ уже вытекает равномерная сходимость последовательности $f_{n}$ внутри области $\widehat{\mathbb{C}} \backslash[\alpha, \beta]$ (в сферической метрике); тем самым, этот ряд определяет аналитическую функцию $f$, допускающую мероморфное продолжение во внешность отрезка $[\alpha, \beta]$ (см. [7], [8]).

Таким образом, диагональные аппроксимации Паде в классе функций вида (8), заданных своими разложениями в степенной ряд (1), полностью решают задачу эффективного аналитического продолэения степенного ряда в область $D=D_{f}$ : полюсы этих аппроксимаций локализуют как отрезок $[\alpha, \beta]=\operatorname{Supp} \sigma$, так и полюсы $f$ в $D$ с учетом их кратностей, а сами аппроксимации $f_{n}$ восстанавливают $f$ внутри $D$.

Из теоремы 1 получаем следующее утверждение о скорости сходимости последовательности $f_{n}$ к функции $f$ в "проколотой" области $D^{\prime}=D \backslash\{$ полюсы $f\}$ :

$$
\varlimsup_{n \rightarrow \infty}\left|\left(f-f_{n}\right)(z)\right|^{1 / n} \leqslant \frac{1}{|\Phi(z)|^{2}},
$$

где $\Phi-$ функция, отображающая внешность отрезка $[\alpha, \beta]$ на внешность единичного круга с условием $\Phi(\infty)=\infty$.

Отсюда уже вытекает $\left[4, \S 2\right.$, п. 10], что полюсы $f_{n}$ стремятся к полюсам $f$ в $D$ со скоростъю геометрической прогрессии

\footnotetext{
${ }^{3}$ Имеется в виду, что за расстояние между двумя точками в комплексной плоскости принимается расстояние между соответствующими точками на сфере Римана.
} 
(в дальнейшем - геометрически); при этом если $a$ - полюс $f$ кратности $\mu \geqslant 1$, то

$$
\varlimsup_{n \rightarrow \infty}\left|a-\zeta_{j, n}(a)\right|^{1 / n} \leqslant \frac{1}{|\Phi(a)|^{2 / \mu}}, \quad j=1, \ldots, \mu, \quad|\Phi(a)|>1
$$

здесь $\zeta_{j, n}(a), j=1, \ldots, \mu,-$ полюсы $^{4} f_{n}$, перенумерованные в порядке неубывания их расстояний до точки $a$.

В работе [4, § 2, п. 11] было отмечено, что как теорема 1, так и ее следствия остаются в силе для любых мероморфных в области $D$ функций $f$ (с конечным числом полюсов в $D$ ), голоморфные составляющие $f^{*}$ которых могут быть представлены в виде

$$
f^{*}(z)=\int_{\alpha}^{\beta} \frac{s(x) d \sigma(x)}{z-x}, \quad z \in D
$$

где $s$ - произвольная комплексная рациональная функция, все нули и полюсы которой принадлежат области $D$. В частности, это относится и к простейшим алгебраическим функциям вида

$$
f(z)=\frac{r_{1}(z)}{w}+r_{2}(z)
$$

где $w=\sqrt{(z-\alpha)(z-\beta)}$, при условии, что все полюсы рациональных функций $r_{1}, r_{2}$ лежат в $D, r_{1}$ не имеет нулей на отрезке $[\alpha, \beta]$ и в $D$ зафиксирована определенная ветвь квадратного корня.

4. В условиях теоремы 1 для функций вида (8) в (9) и (10) имеет место точное равенство; тем самым, в правой части (10) указан правильный показатель скорости сходимости полюсов $f_{n}$ к полюсам $f$ в области $D$ (ср. [7], а также $\S 2$, п. 3 ниже). В то же время соотношения такого типа дают информацию о поведении каждого из $\mu$ полюсов аппроксимаций Паде по отдельности и не позволяют судить о том, какова "в целом" картина поведения $\mu$ полюсов $f_{n}$ в окрестности $\mu$-кратного полюса $f$. Для выяснения возможных закономерностей в асимптотическом поведении полюсов аппроксимаций Паде $f_{n}$ в окрестности кратного полюса $f$ мы провели соответствующие вычисления для конкретных примеров

\footnotetext{
${ }^{4}$ Как обычно, полюсы и нули функций считаются с учетом их кратностей.
} 
функций вида (11). С помощью программы, написанной на языке FORTRAN и работающей под управлением операционной системы LINUX, были проведены вычисления (с точностью в 40 значащих цифр) нулей и полюсов диагональных аппроксимаций Паде для функций вида

$$
f(z)=\frac{1}{\sqrt{z^{2}-1}}+r(z)
$$

где $r$ - вещественная рациональная функция, которая в одном случае имеет единственный полюс 10 -й кратности в точке $a>1$, a в другом - два полюса в точках $a$ и $\bar{a}, \operatorname{Im} a>0$, каждый 10-й кратности. В обоих случаях ветвь корня в (12) выбиралась так, что $\sqrt{z^{2}-1}>0$ при $z>1$.

Результаты численного анализа рассматриваемой задачи (для достаточно больших $n$ ) представлены на рис. 1-8. Сделаем необходимые пояснения. На рис. 1 изображены нули (белые кружки) и полюсы (черные кружки) диагональной аппроксимации Паде $f_{28}$ функции вида (12) для первого случая с $a=1.45$. Часть нулей и полюсов локализует отрезок $\Delta=[-1,1]$, при этом они перемежаются между собой. Остальные нули рациональной функции $f_{28}$ локализуют простые нули функции $f$ в области $D$. То, что эти нули простые, отчетливо видно из сравнения рис. 1 и рис. 3: pacположение таких нулей для аппроксимаций Паде $f_{n}$ очень быстро стабилизируется и при последовательных $n=21,22, \ldots, 33$ остается практически неизменным, так как в соответствии с (10) они стремятся к нулям $f$ в $D$ геометрически ${ }^{5}$ (отметим, что расстояние между такими нулями $f_{n}$, локализующими простой нуль $f$, при двух последовательных значениях $n=42,43$ уже оказывается порядка $\left.10^{-10}\right)$. Такой же характер имеет сходимость полюсов $f_{n}$ к простому полюсу $f$.

Иначе обстоит дело в случае кратного (здесь - 10-кратного) полюса $f$ : как видно из рис. 2 , все 10 полюсов $f_{n}$ в окрестности этого полюса простые; они по-прежнему сходятся к полюсу $f$ геометрически (см. рис. 3 и 4), но теперь в соответствии с формулой (10) общий показатель скорости сходимости $|\Phi(a)|^{-2}<1$ распределяется между 10-ю простыми полюсами рациональной дроби $f_{n}$, локализующими точку $z=a$. Более того, это распределение

\footnotetext{
5 Это вытекает из очевидной связи между диагональными аппроксимациями Паде для функций $f$ и $1 / f$.
} 
носит равномерный характер, а совокупность из этих 10-ти простых полюсов $f_{n}$ ведет себя регулярно в целом: асимптотически они располагаются в вершинах правильного 10-угольника. В этом убеждают результаты расчетов, представленные на рис. 2, 4, 6, 8 .

Основная цель настоящей работы - вывод формул сильной асимптотики (см. $\left.\left(22_{1}\right)-\left(22_{3}\right)\right)$ для знаменателей диагональных аппроксимаций Паде мероморфных функций марковского типа $f=\widehat{\sigma}+r$ при дополнительных ограничениях на $\sigma$ (см. ниже $\S 2)$. На основе соответствующих формул в работе доказано, что в достаточно малой окрестности кратного полюса мероморфной функции $f=\widehat{\sigma}+r$ все полюсы рациональных функций $f_{n}$ простые и ведут себя именно так, как было указано выше в связи с проведенными численными расчетами.

Доказательство этих результатов основано на свойствах $\Psi$-функции, решающей специальную краевую задачу Римана на двулистной римановой поверхности. В работе рассмотрен также случай, когда носитель $\sigma$ состоит из нескольких отрезков.

Настоящая работа выполнена в рамках Программы РАН "Современные проблемы теоретической математики" и Программы поддержки ведущих научных школ России (грант № НШ-1551.2003.1), а также при поддержке Российского фонда фундаментальных исследований (грант № 02-01-00564) и фонда INTAS (грант № 03-51-6637). 


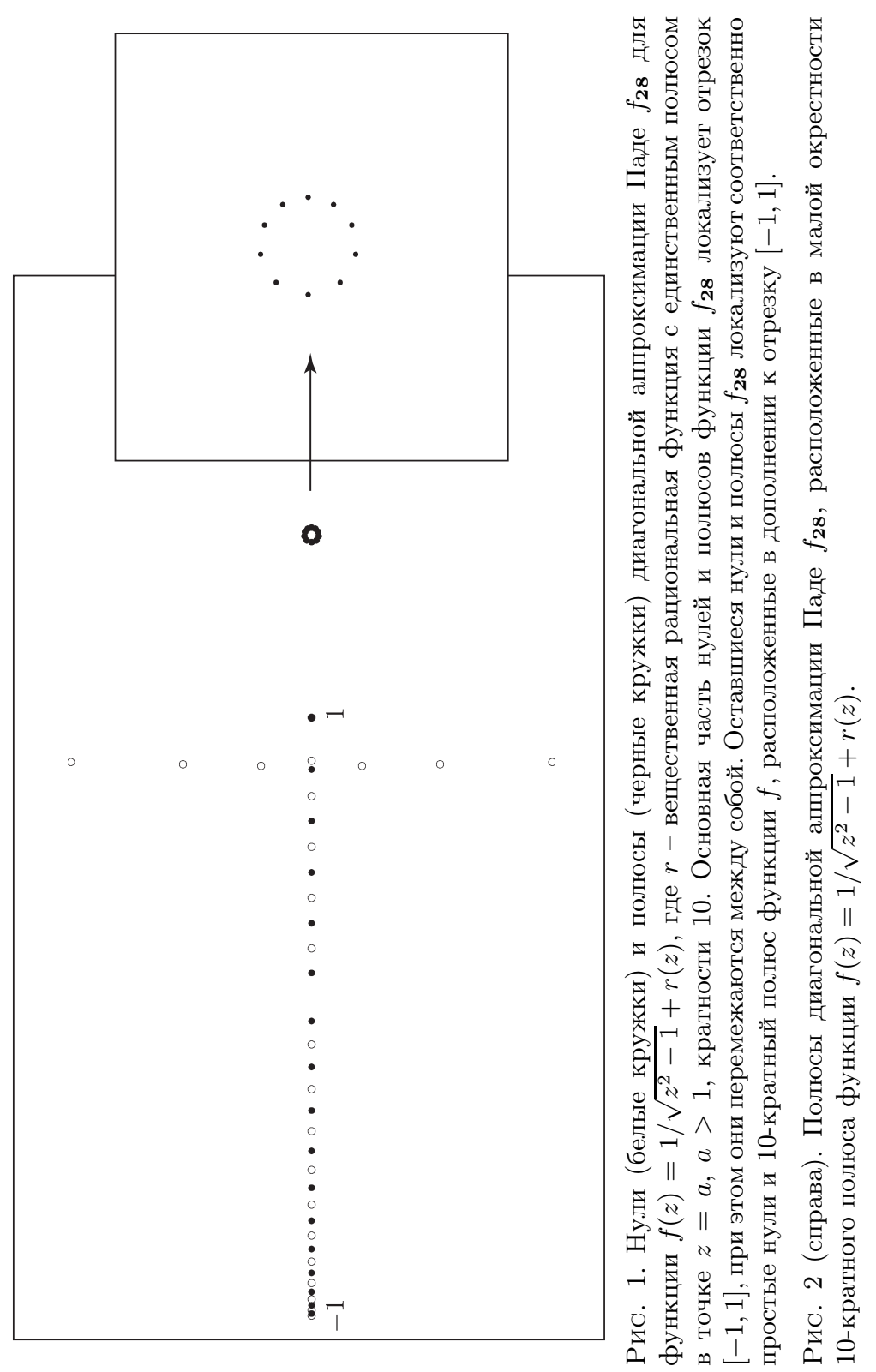



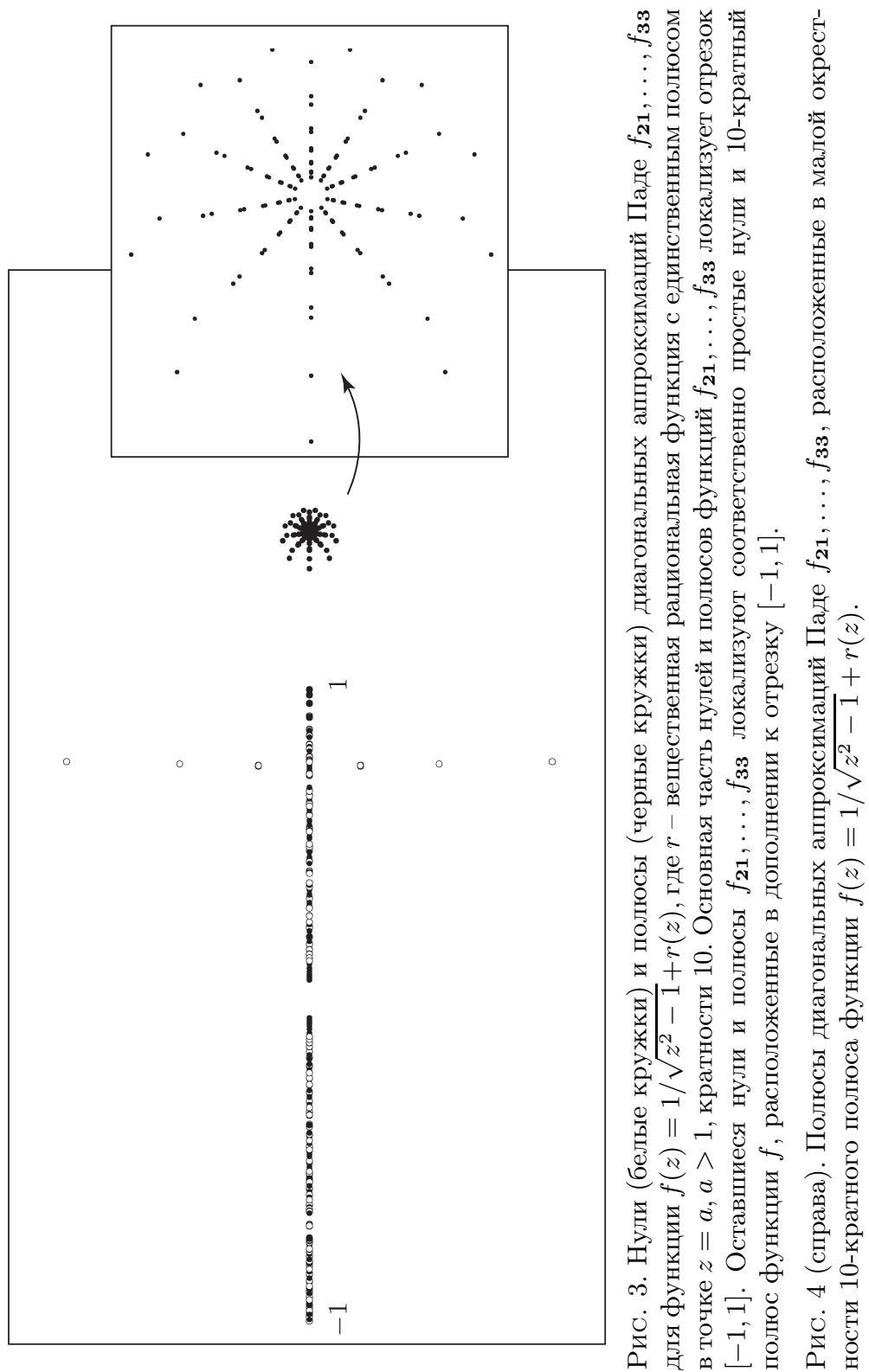


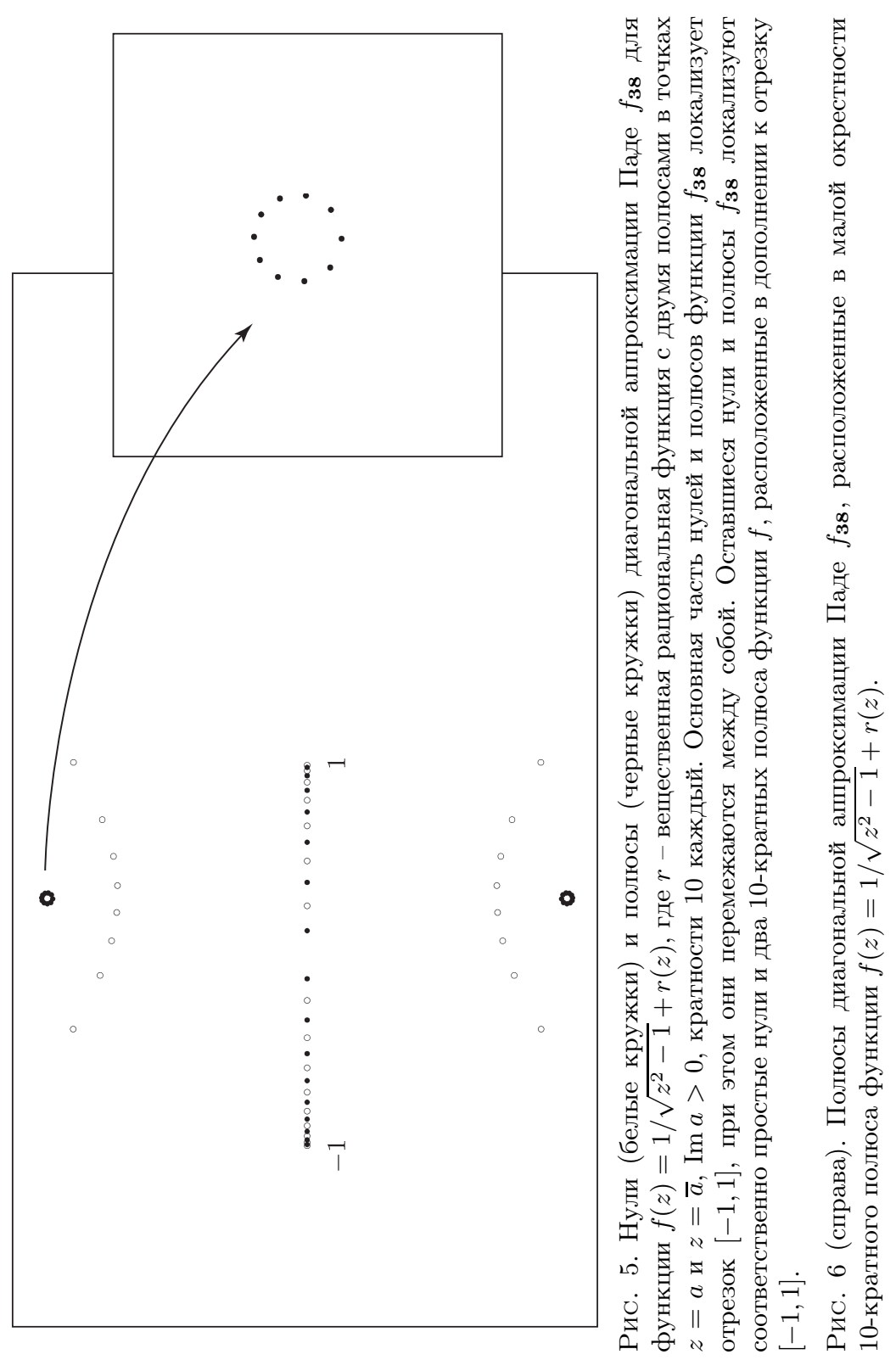




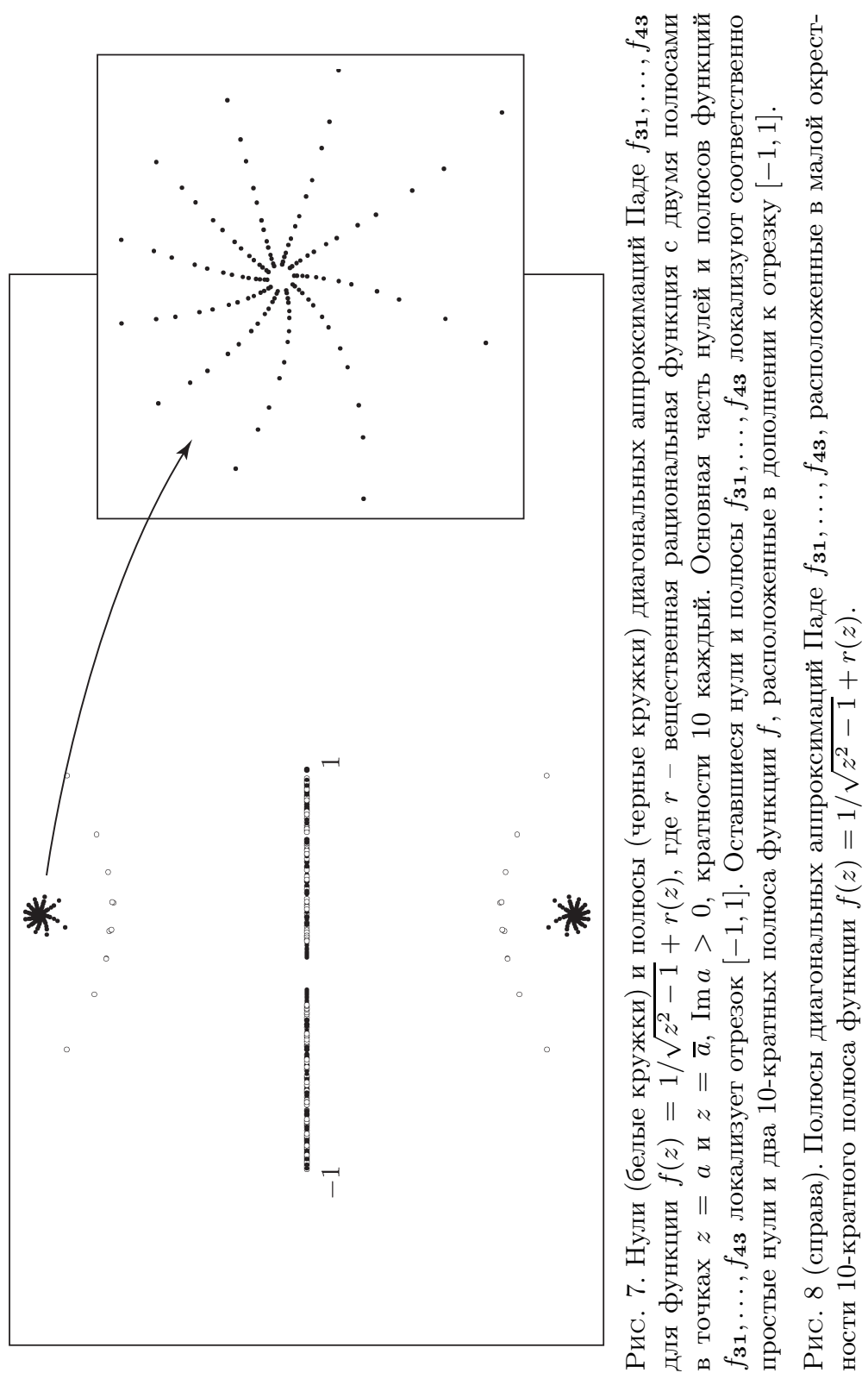




\section{§ 2. Формулировка основных результатов}

1. Всюду в дальнейшем полагаем $\Delta=[-1,1], \quad D=\widehat{\mathbb{C}} \backslash \Delta$. Мы будем рассматривать здесь класс функиий вида

$$
f=\widehat{\rho}+r,
$$

где $\mathrm{e}^{6}$

$$
\widehat{\rho}(z):=\frac{1}{\pi} \int_{\Delta} \frac{\rho(x)}{z-x} \frac{d x}{\sqrt{1-x^{2}}},
$$

комплекснозначная функция $\rho$ голоморфна на отрезке $\Delta$ и не обращается в нуль на этом отрезке, все полюсы комплексной рациональной функции $r$ лежат в $D$ и $r(\infty)=0$. По традиции, восходящей к С. Н. Бернштейну [9], функцию $\rho$ называют тригонометрическим весом; для краткости мы будем называть $\rho$ просто весом. Очевидно, что класс функций (13) содержит функции вида (11) с $w=\sqrt{z^{2}-1}$ и $r_{1} \neq 0, \infty$ на $\Delta$. Тем самым, все результаты настоящей работы справедливы и для таких простейших алгебраических функций.

В основе доказательства теоремы 1 в работе [4] лежит формула сравнительной асимптотики полиномов $Q_{n}(z ; \widehat{\sigma}+r)$ и $Q_{n}(z ; \widehat{\sigma})$ вне отрезка $\Delta$. Такая же формула (см. (20)) доказывается ниже для полиномов $Q_{n}(z ; f)$ и $Q_{n}(z ; \widehat{\rho})$ - знаменателей диагональных аппроксимаций Паде функций вида (13) и (14). Эта формула является ключевой при доказательстве теоремы 2 - аналога теоремы 1 - и уже сама может рассматриваться как формула сильной асимптотики для полиномов $Q_{n}(z ; f)$, но только внутри облаcmu $D^{\prime}=D \backslash\{$ полюсы $f\}$. В настоящей работе для исследования сильной асимптотики полюсов диагональных аппроксимаций Паде функций вида $f=\widehat{\rho}+r$ в области $D$ нам понадобится сильная асимптотика полиномов $Q_{n}(z ; f)$ как вне отрезка $\Delta$, так и на самом отрезке. Эти формулы сильной асимптотики будут получены в терминах, связанных с римановой поверхностью $\mathfrak{R}: w^{2}=z^{2}-1$. Прежде чем формулировать основные результаты настоящей работы, приведем необходимые определения и обозначения, связанными с простейшими и хорошо известными конструкциями.

\footnotetext{
${ }^{6}$ Используемые нами обозначения $\widehat{\sigma}$ и $\widehat{\rho}$ для меры $\sigma$ и функции $\rho$ имеют разный смысл; ср. формулы (5) и (14). Однако из текста работы всегда ясно, какой из двух случаев имеется в виду.
} 
Пусть $\mathfrak{R}$ - риманова поверхность, заданная уравнением $w^{2}=$ $z^{2}-1$. Будем считать, что $\mathfrak{R}$ реализована как двулистное разветвленное в точках $z= \pm 1$ накрытие расширенной комплексной плоскости $\widehat{\mathbb{C}}$. Два листа $\mathfrak{R}-$ это два экземпляра $\widehat{\mathbb{C}}$, разрезанных по отрезку $\Delta$ и склеенных затем накрест так, что верхнему берегу одного отрезка соответствует нижний берег другого. Тем самым, над каждой точкой $\widehat{\mathbb{C}}$ за исключением точек ветвления $z= \pm 1$ лежат ровно две точки римановой поверхности, а отрезку $\Delta$ соответствует на $\mathfrak{R}$ замкнутый цикл $Г$. Будем считать, что в $D=\widehat{\mathbb{C}} \backslash \Delta$ выбрана ветвь корня $\sqrt{z^{2}-1}$, удовлетворяющая условию $\sqrt{z^{2}-1} / z \rightarrow 1$ при $z \rightarrow \infty$. Функция $w: w^{2}=z^{2}-1$ однозначна на $\mathfrak{R}$. Первым (открытым) листом $D^{(1)}$ поверхности $\mathfrak{R}$ будем считать тот, на котором $w=\sqrt{z^{2}-1}$. На втором листе $D^{(2)}$ имеем: $w=-\sqrt{z^{2}-1}$. Для точек римановой поверхности $\Re$ будем использовать обозначение $\mathbf{z}=(z, w)$, где $w= \pm \sqrt{z^{2}-1} ;$ при этом для точек первого листа $z^{(1)}=\left(z, \sqrt{z^{2}-1}\right)$, а для точек второго $z^{(2)}=\left(z,-\sqrt{z^{2}-1}\right)$. Область $D^{(1)}$ будем, как правило, отождествлять с "физической” областью $D$. Пусть $\Phi(z)=z+\sqrt{z^{2}-1}-$ функция, обратная к функции Жуковского. Тогда $\Phi$ однозначна в $D$ и продолжается на всю риманову поверхность $\Re$ как однозначная мероморфная функция, $\Phi(\mathbf{z})=z+w \in \mathbb{C}(z, w)$, с дивизором $(\Phi)=\infty^{(2)}-\infty^{(1)}$.

Риманова поверхность $\mathfrak{R}$ - нулевого рода, поэтому на $\mathfrak{R}$ разрешим любой дивизор нулевой степени. Для дивизора вида $a^{(1)}-b^{(2)}, \quad$ где $a, b \in \mathbb{C} \backslash \Delta$, легко указать явно функцию $\Phi_{a, b} \in \mathbb{C}(z, w)$ такую, что $\left(\Phi_{a, b}\right)=a^{(1)}-b^{(2)}$ :

$$
\Phi_{a, b}(\mathbf{z})=\frac{\Phi(\mathbf{z})-\Phi(b)}{z-b} \frac{z-a}{1-\Phi(a) \Phi(\mathbf{z})} ;
$$

при $a=\infty$ или $b=\infty$ формула для соответствующей функции $\Phi_{a, b}$ получается предельным переходом в (15). Очевидно, что произвольному дивизору нулевой степени на $\mathfrak{R}$ будет соответствовать произведение функций вида (15).

Функции Грина $g_{D}(z, \infty)=\log |\Phi(z)|$ области $D$ соответствует комплексная функция Грина $G_{D}(z, \infty)=g_{D}(z, \infty)+i g_{D}^{*}(z, \infty)$, где $g_{D}^{*}(z, \infty)$ - гармонически сопряженная к $g_{D}(z, \infty)$ функция. Функция $G(z)=G_{D}(z, \infty)$ имеет в бесконечно удаленной точке ветвление логарифмического характера, однозначна по модулю $2 \pi i$ и представляется в виде

$$
G(z)=\log \Phi(z)=\int_{1}^{z} \frac{d \zeta}{\sqrt{\zeta^{2}-1}}, \quad \zeta \in D
$$


где интегрирование - по любому пути, лежащему в $D$.

Используемый здесь подход к изучению асимптотических свойств знаменателей диагональных аппроксимаций Паде $Q_{n}(z ; f)$ основан на свойствах $\Psi$-функции, решающей специальную краевую задачу Римана на двулистной римановой поверхности $\Re$. Такой подход берет начало с работ Н. И. Ахиезера [10]-[12] и был затем развит в работах Дж. Наттолла [13], [14] и С. П. Суетина [15]-[17] прежде всего - с целью изучения вопросов сходимости диагональных аппроксимаций Паде для гиперэллиптических функций и их естественных обобщений. Отметим также работу А.И. Аптекарева [18], посвященную вопросам изучения сильной асимптотики рациональных аппроксимаций аналитических функций, где применяется метод, основанный, в частности, на свойствах решения краевой задачи Римана подобного типа.

Как правило, наиболее общее условие на вес $\rho$ для применимости такого подхода - это условие Гёльдера. В настоящей работе для простоты мы изучаем случай, когда вес $\rho-$ голоморфная функция на отрезке $\Delta(\rho \in \mathcal{H}(\Delta))$.

2. Рассмотрим сначала ряд (1) для функции $f=\widehat{\rho}$, где $\rho \in$ $\mathcal{H}(\Delta)$ и $\rho(x) \neq 0$ на $\Delta$. Зафиксируем произвольное $n \in \mathbb{N}$ и рассмотрим на римановой поверхности $\mathfrak{R}$ следующую краевую задачу Римана.

ЗАДАчА $\left(\mathcal{R}_{1}\right)$. При фиксированном $n \in \mathbb{N}$ найти функиию $\Psi=\Psi_{n}$ такую, что:

$1^{\circ}$. $\Psi$ (кусочно) мероморфна на $\Re \backslash \Gamma=D^{(1)} \sqcup D^{(2)}$;

$2^{\circ}$. дивизор $(\Psi)=n \infty^{(2)}-n \infty^{(1)}$;

$3^{\circ}$. на Г выполнено краевое условие: $\rho(x) \Psi(\mathbf{x}+)=\Psi(\mathbf{x}-)$.

Здесь под $\Psi(\mathbf{x}+)$ понимаются предельные значения функции $\Psi(\boldsymbol{\zeta})$ при $D^{(1)} \ni \boldsymbol{\zeta} \rightarrow \mathbf{x} \in \Gamma$. Аналогичный смысл придается и $\Psi(\mathbf{x}-)$.

Так как род $\mathfrak{R}$ равен нулю и $\operatorname{deg}(\Psi)=0$, то задача $\left(\mathcal{R}_{1}\right)$ распадается на две независимые задачи, соответствующие п. $2^{\circ}$ и п. $3^{\circ}$. Стандартными методами (см., например, [16]) доказывается, что такая функция $\Psi$ всегда существует и определяется однозначно с точностью до мультипликативной постоянной. Нетрудно найти и явный вид этой функции:

$$
\Psi(z)=\Phi(z)^{n} e^{S(z)}=e^{n G(z)+S(z)} \quad \text { при } \quad \mathbf{z}=z^{(1)},
$$


где

$$
S(z)=\frac{\sqrt{z^{2}-1}}{2 \pi} \int_{\Delta} \frac{\log \rho(x)}{z-x} \frac{d x}{\sqrt{1-x^{2}}}
$$

- функция Сегё. Из свойств $\Psi$-функции вытекает, что

$$
\Psi\left(z^{(1)}\right) \Psi\left(z^{(2)}\right) \equiv \text { const } \neq 0
$$

при $z \in D$. Тем самым, это произведение продолжается как постоянная функция на всю комплексную плоскость $\widehat{\mathbb{C}}$. В дальнейшем нам удобно ввести следующую нормировку ${ }^{7}: \Psi\left(z^{(1)}\right) \Psi\left(z^{(2)}\right) \equiv 1$ при $z \in D$. Последнее соотношение в совокупности с (16), (17) приводит к явной формуле для $\Psi$-функции и при $\mathbf{z}=z^{(2)} \in D^{(2)}$.

При подходящей нормировке (подробнее см. ниже $§ 3$, п. 3) полиномов $Q_{n}(z ; \widehat{\rho})$ - знаменателей диагональных аппроксимаций Паде функции $f=\widehat{\rho}$ - их сильная асимптотика как вне отрезка $\Delta$, так и на этом отрезке описывается в терминах $\Psi$-функции следующим образом.

ПРЕДЛОЖЕНИЕ 1. Пусть комплекснозначная функиия $\rho$ голоморфна и не обращается в нуль на отрезке $\Delta, \Psi$ - решение задачи $\left(\mathcal{R}_{1}\right)$ при $n \in \mathbb{N}$. Тогда при подходящей нормировке полиномов $Q_{n}(z ; \widehat{\rho})$ имеем:

$$
\begin{aligned}
& 1^{\circ} . Q_{n}(z ; \widehat{\rho})=\Psi(z)(1+o(1)) \text { при } n \rightarrow \infty \text { равномерно внутри } D ; \\
& 2^{\circ} . Q_{n}(x ; \widehat{\rho})=\Psi^{+}(x)+\Psi^{-}(x)+o(1) \text { при } n \rightarrow \infty \text { равномерно } \\
& \quad \text { на } \Delta .
\end{aligned}
$$

Здесь $\Psi(z)=\Psi\left(z^{(1)}\right)$, под $\Psi^{+}(x) \quad\left(\Psi^{-}(x)\right)$ понимаются верхние (соответственно нижние) предельные значения $\Psi$ на $\Delta$; величина $o(1)=o\left(\delta^{n}\right)$, где $\delta<1$. Из п. $2^{\circ}$ задачи $\left(\mathcal{R}_{1}\right)$ вытекает, что функция $\Psi$, решающая эту задачу, имеет в бесконечно удаленной точке $\mathbf{z}=\infty^{(1)}$ полюс $n$-го порядка. Поэтому в силу п. $1^{\circ}$ предложения 1 для всех достаточно больших $n$ степень полинома $Q_{n}(z ; \widehat{\rho})$ равна $n$, т.е. все такие индексы нормальны. Непосредственно из представления (16) для $\Psi$-функции вытекает, что существует

$$
\lim _{z \rightarrow \infty} \frac{\Psi(z)}{z^{n}}=\varkappa_{n}, \quad \text { где } \varkappa_{n}=2^{n} e^{S(\infty)} \neq 0 .
$$

\footnotetext{
${ }^{7}$ Таким условием $\Psi$-функция определяется однозначно с точностью до знака \pm . В дальнейшем мы уточним правило выбора знака.
} 
Тем самым, формула сильной асимптотики из п. $1^{\circ}$ задает следующую естественную нормировку полиномов $Q_{n}$ :

$$
Q_{n}(z ; \widehat{\rho})=\varkappa_{n} z^{n}+\cdots,
$$

где $\varkappa_{n} \neq 0$ задано (18). Наконец, из п. $2^{\circ}$ вытекает, что

$$
\int_{\Delta} Q_{n}^{2}(x ; \widehat{\rho}) \frac{\rho(x) d x}{\sqrt{1-x^{2}}}=2+o(1) \quad \text { при } n \rightarrow \infty,
$$

а если вес $\rho$ положителен на $\Delta$, то равномерно на $\Delta$

$$
Q_{n}(x ; \widehat{\rho})=\frac{2}{\sqrt{\rho(x)}} \cos (n \arccos x+\theta(x))+o(1)
$$

где

$$
\theta(x)=\frac{\sqrt{1-x^{2}}}{2 \pi} \int_{\Delta} \frac{\log \rho(t)-\log \rho(x)}{x-t} \frac{d t}{\sqrt{1-t^{2}}}, \quad o(1)=o\left(\delta^{n}\right) .
$$

Предложение 1 и его следствия хорошо известны (см., например, [13] и [14]). В §3 настоящей работы для полноты изложения приводится его независимое доказательство (ср. с доказательством теоремы 2.1 в [14]).

3. Прежде чем переходить непосредственно к вопросу о сильной асимптотике полиномов $Q_{n}(z ; f), f=\widehat{\rho}+r$, сформулируем аналог теоремы 1 для изучаемого нами случая.

ТЕОРема 2. Пусть $f=\widehat{\rho}+r$, где комплекснозначная функиия $\rho$ голоморбна и не обращается в нуль на отрезке $\Delta$, все полюсы комплексной рационалъной функиии $r$ лежст в области $D$ $u r(\infty)=0$. Тогда последовательность диагональных аппроксимаций Паде $\left\{f_{n}\right\}_{n \in \mathbb{N}}$ сходится $\kappa f$ равномерно в сферической метрике внутри области $D$.

Тем самым, к каждому полюсу функции $f$ в $D$ стремится ровно столько полюсов $f_{n}$, какова его кратность. При этом если $a-$ полюс $f$ кратности $\mu \geqslant 1$, то

$$
\varlimsup_{n \rightarrow \infty}\left|a-\zeta_{j, n}(a)\right|^{1 / n} \leqslant \frac{1}{|\Phi(a)|^{2 / \mu}}<1, \quad j=1, \ldots, \mu
$$

здесь $\Phi(z)=z+\sqrt{z^{2}-1}-$ функция, обратная к функции Жуковского, $\zeta_{j, n}(a), j=1, \ldots, \mu$, - полюсы $f_{n}$, перенумерованные в порядке неубывания их расстояния до точки $a$. 
Доказательство теоремы 2 проводится (см. ниже §4) по той же схеме, что и доказательство теоремы 1 в работе [4]; в частности, утверждение об асимптотике полюсов $f_{n}$ в $D$ немедленно следует из полученной в $\S 4$ формулы сравнительной асимптотики для подходящим образом нормированных полиномов $Q_{n}(z ; \widehat{\rho})$ и $Q_{n}(z ; \widehat{\rho}+r)$, которая выполняется равномерно внутри $D$ :

$$
\lim _{\substack{n \rightarrow \infty \\ n \in \Lambda}} \frac{Q_{n}(z ; \widehat{\rho}+r)}{Q_{n}(z ; \hat{\rho})}=\prod_{j=1}^{\ell}\left(\frac{\Phi(z)-\Phi\left(a_{j}\right)}{1-\Phi(z) \Phi\left(a_{j}\right)}\right)^{m_{j}}
$$

где $\Lambda=\Lambda(f)$ - последовательность нормальных индексов для $f=\widehat{\rho}+r, a_{1}, \ldots, a_{\ell}-$ полюсы $f$ в $D, m_{1}, \ldots, m_{\ell}-$ их кратности, $m=\sum_{j=1}^{\ell} m_{j}$ - полное число полюсов $f$ в $D$. Соотношение (20) является ключевым и при доказательстве теоремы 2; с учетом предложения 1 оно само уже может рассматриваться как формула сильной асимптотики для полиномов $Q_{n}(z ; f)$, но - по подпоследовательности нормальных индексов $\Lambda$ и только внутри области $D^{\prime}=D \backslash\left\{a_{1}, \ldots, a_{\ell}\right\}$. Для доказательства основного результата работы (см. ниже теорему 3 ) нам, во-первых, надо показать, что все достаточно далекие индексы $n$ нормальны, а во-вторых, установить формулу сильной асимптотики для полиномов $Q_{n}(z ; f)$ не только внутри $D^{\prime}$, но и на отрезке $\Delta$, и, в конечном счете, в окрестности полюсов функции $f$ в $D$. Для этого мы применим подход, основанный, как и в случае полиномов $Q_{n}(z ; \widehat{\rho})$, на свойствах $\Psi$-функции, решающей специальную краевую задачу Римана на римановой поверхности $\Re$. Подчеркнем, что сильную асимптотику $Q_{n}(z ; f)$ можно выразить непосредственно в терминах этой $\Psi$-функции только внутри области $D^{\prime}=D \backslash\left\{a_{1}, \ldots, a_{\ell}\right\}$ и на отрезке $\Delta$. Зато, пользуясь этими результатами, мы затем найдем сильную асимптотику тех нулей $Q_{n}(z ; f)$ (т.е. полюсов $\left.f_{n}\right)$, которые стремятся к полюсам $f$ в $D$. Полученные в итоге формулы (см. ниже формулы $\left(22_{1}\right)-\left(22_{3}\right)$ ) и следует в совокупности рассматривать как формулы сильной асимптотики для $Q_{n}(z ; f)$.

Рассмотрим на римановой поверхности $\mathfrak{R}$ следующую краевую задачу Римана. 
ЗАДАчА $\left(\mathcal{R}_{2}\right)$. При фиксированном $n \in \mathbb{N}$ найти функцию $\Psi=\Psi_{n}$ такую, что:

$1^{\circ}$. $\Psi$ (кусочно) мероморфнан на $\mathfrak{R} \backslash \Gamma=D^{(1)} \sqcup D^{(2)}$;

$2^{\circ}$. дивизор $(\Psi)=n \infty^{(2)}+m_{1} a_{1}^{(1)}+\cdots+m_{\ell} a_{\ell}^{(1)}-m_{1} a_{1}^{(2)}-\cdots-$ $m_{\ell} a_{\ell}^{(2)}-n \infty^{(1)}$

$3^{\circ}$. на Г выполнено краевое условие: $\rho(x) \Psi(\mathbf{x}+)=\Psi(\mathbf{x}-)$.

(Здесь предполагается, что все точки $a_{j} \in \mathbb{C} \backslash \Delta, j=1, \ldots, \ell$.)

Подчеркнем еще раз, что непосредственно в терминах $\Psi$-функции, решающей задачу $\left(\mathcal{R}_{2}\right)$, оказывается возможным описать сильную асимптотику $Q_{n}(z, f)$ как на отрезке $\Delta$, так и внутри области $D^{\prime}=D \backslash\left\{a_{1}, \ldots, a_{\ell}\right\}$.

Так как в рассматриваемом здесь случае род римановой поверхности $\mathfrak{R}$ равен нулю и $\operatorname{deg}(\Psi)=0$, то задача $\left(\mathcal{R}_{2}\right)$, как и задача $\left(\mathcal{R}_{1}\right)$, распадается на независимые задачи, соответствующие п. $2^{\circ}$ и п. $3^{\circ}$. Каждая из них решается явно в терминах, связанных с $\mathfrak{R}$. Таким образом, решение задачи $\left(\mathcal{R}_{2}\right)$ всегда существует, единственно (с точностью до мультипликативной постоянной) и может быть найдено в явном виде. Зависимость $\Psi$-функции от $n$ связана только с условием $2^{\circ}$. Нетрудно увидеть, что при $z \in D$ функция $\Psi\left(z^{(1)}\right) \Psi\left(z^{(2)}\right) \equiv$ const, где const $\neq 0$. В дальнейшем нам удобно как обычно положить const $=1$, т.е. нормировать $\Psi$-функцию (как и ранее, с точностью до знака \pm ) стандартным условием

$$
\Psi\left(z^{(1)}\right) \Psi\left(z^{(2)}\right) \equiv 1 .
$$

Приведем явный вид решения задачи $\left(\mathcal{R}_{2}\right)$ при $\mathbf{z}=z^{(1)} \in D^{(1)}$ :

$$
\Psi(z)=\Phi(z)^{n} e^{S(z)} \prod_{j=1}^{\ell}\left(\frac{\Phi(z)-\Phi\left(a_{j}\right)}{1-\Phi\left(a_{j}\right) \Phi(z)}\right)^{m_{j}}=e^{n G(z)+S(z)} \Pi_{m}(z),
$$

где $S(z)$ - функция Сегё (17).

В терминах $\Psi$-функции, решающей задачу $\left(\mathcal{R}_{2}\right)$, асимптотическое поведение полиномов $Q_{n}(z ; f)$ описывается следующим образом.

ПРЕДЛОЖЕНИЕ 2. Пусть в условиях теоремы 2 точки $a_{1}$, $\ldots, a_{\ell}-$ полюсы $f$ в $D, m_{1}, \ldots, m_{\ell}-$ их кратности, $\Psi$ - решение задачи $\left(\mathcal{R}_{2}\right)$ при $n \in \mathbb{N}$. Тогда при подходящей нормировке полиномов $Q_{n}(z ; f)$ имеем: 
$1^{\circ} . Q_{n}(z ; f)=\Psi(z)(1+o(1))$ при $n \rightarrow \infty$ равномерно внутри $D^{\prime}$,

$2^{\circ} \cdot Q_{n}(x ; f)=\Psi^{+}(x)+\Psi^{-}(x)+o(1)$ при $n \rightarrow \infty$ равномерно на $\Delta$,

где $D^{\prime}=D \backslash\left\{a_{1}, \ldots, a_{\ell}\right\}$, величина $о(1)=o\left(\delta^{n}\right), \delta<1$.

Непосредственно из п. $1^{\circ}$ этого предложения и свойств $\Psi$-функции, решающей задачу $\left(\mathcal{R}_{2}\right)$, вытекает, что $\operatorname{deg} Q_{n}(z ; f)=n$ для достаточно больших $n$, т.е. для функции $f$ все достаточно большие индексы $п$ нормальны.

Полученная в п. $2^{\circ}$ предложения 2 формула сильной асимптотики полиномов $Q_{n}(x ; f)$ на отрезке $\Delta$ лежит в основе доказательства следующего утверждения об асимптотическом поведении полюсов $f_{n}$ вне отрезка $\Delta$.

Теорема 3. Пусть в условиях теоремы 2 точка $a_{j} \in D-$ полюс функиии $f$ кратности $m_{j} \geqslant 1, a_{j, \nu}(n), \nu=1, \ldots, m_{j},-$ соответствующие точке $a_{j}$ при $n \rightarrow \infty$ полюсъ $f_{n}$. Тогда

$$
a_{j, \nu}(n)=a_{j}+\frac{C_{j} \varepsilon_{j}^{\nu}}{B_{j}^{2 n}}\left(1+o\left(\delta^{n}\right)\right) \quad n p u \quad n \rightarrow \infty, \nu=1, \ldots, m_{j},
$$

где

$$
\begin{aligned}
\varepsilon_{j}= & e^{2 \pi i / m_{j}}, \\
B_{j}= & \Phi\left(a_{j}\right)^{1 / m_{j}}=\left|\Phi\left(a_{j}\right)\right|^{1 / m_{j}} e^{i g^{*}\left(a_{j}\right) / m_{j}}, \\
C_{j}=e^{-2 S\left(a_{j}\right) / m_{j}}\left(-\frac{2 G^{\prime}\left(a_{j}\right)}{A_{j} \alpha_{j}}\right)^{1 / m_{j}} & \\
& \quad \times \frac{(-2)^{m / m_{j}} \Phi\left(a_{j}\right)^{m / m_{j}} \prod_{s=1}^{\ell} \Phi\left(a_{s}\right)^{m_{s} / m_{j}}}{\prod_{s=1}^{\ell}\left(1-\Phi\left(a_{s}\right) \Phi\left(a_{j}\right)\right)^{2 m_{s} / m_{j}}} ;
\end{aligned}
$$

комплексные постоянные $A_{j} u \alpha_{j}, j=1, \ldots, \ell$, конечны, отличны от нуля и зависят только от $f$.

Здесь $g^{*}(z)=g_{D}^{*}(z, \infty)$ - функция, сопряженная к функции Грина $g_{D}(z, \infty)$ области $D, \quad G(z)=G_{D}(z, \infty)$ - комплексная функция Грина; $S(z)$ - функция Сегё.

Постоянная $C_{j}$ связана с геометрическим расположением $j$-го полюса $a_{j}$ функции $f$ относительно отрезка $\Delta$ и других полюсов $f$; величина $1 / B_{j}$ характеризует (геометрическую) скорость 
сходимости полюсов $f_{n}$ к точке $a_{j}$ и не зависит от других полюсов $f$; наличие множителя $\varepsilon_{j}, \varepsilon_{j}^{m_{j}}=1$, обеспечивает то асимптотически правильное расположение $m_{j}$ полюсов $f_{n}$ по отношению к притягивающей точке $a_{j}$, о котором говорилось во введении. Непосредственно из $\left(22_{3}\right)$ вытекает, что при каждом $j=1, \ldots, \ell$ существует

$$
\lim _{n \rightarrow \infty}\left|a_{j}-a_{j, \nu}(n)\right|^{1 / n}=\frac{1}{\left|\Phi\left(a_{j}\right)\right|^{2 / m_{j}}}, \quad \nu=1, \ldots, m_{j}
$$

тем самым, в правой части (10) указан правильный показатель скорости сходимости полюсов $f_{n}$ к полюсам $f$ в $D$.

Из теоремы 3 вытекают следующие результаты.

СлЕДСтвиЕ 1. В классе функиий вида $f=\widehat{\rho}+r$, где функиия $\rho$ голоморфна и не обращается в нуль на отрезке $\Delta$, а все полюсы комплексной рациональной функции $r$ лежат в области $D$ и $r(\infty)=0$, справедлива импликация

$$
\begin{gathered}
\left(f-f_{n}\right)(z)=\frac{A}{z^{2 n+1}}+\cdots \\
\Downarrow \\
\left(f-f_{n}\right)(z)=\frac{2 G^{\prime}(z)}{\Psi(z)^{2}}(1+o(1)), \quad n \rightarrow \infty, \\
\text { равномерно внутри } D^{\prime},
\end{gathered}
$$

где $f_{n}, n \in \mathbb{N},-$ рациональная функиия порядка не выше $n$, о $(1)=$ $o\left(\delta^{n}\right), \quad \delta<1$.

Соотношение (23) приводит к формуле сильной асимптотики для последовательности $\left\{f_{n}\right\}_{n \in \mathbb{N}}$ :

$$
\begin{gathered}
f_{n}(z)=f(z)-\frac{2 G^{\prime}(z)}{\Psi(z)^{2}}(1+o(1)) \\
\text { равномерно внутри } D^{\prime} \text { при } n \rightarrow \infty .
\end{gathered}
$$

СЛЕДСТВИЕ 2. Пусть в условиях теоремъ 2 вес $\rho$ положителен на $\Delta$, а рациональная функиия $r$ вещественна. Тогда для всех достаточно больших $n$ все полюсы диагональных аппроксимаций Паде функции $f=\widehat{\rho}+r$ простые. 
СЛЕДСТВИЕ 3. Пусть в условиях теоремы 2 вес $\rho>0$ на $\Delta$, $f=\widehat{\rho}+r, r \in \mathbb{R}(z)$. Тогда равномерно на $\Delta$

$Q_{n}(x ; f)=\frac{2}{\sqrt{\rho(x)}} \cos (n \arccos x+\theta(x)+\alpha(x))+o\left(\delta^{n}\right), \quad n \rightarrow \infty$, $2 \partial e$

$$
\begin{aligned}
\theta(x)= & \frac{\sqrt{1-x^{2}}}{2 \pi} \int_{\Delta} \frac{\log \rho(t)-\log \rho(x)}{x-t} \frac{d t}{\sqrt{1-t^{2}}}, \\
\cos \alpha(x)= & \frac{1}{2}\left\{\prod_{j=1}^{\ell}\left(\frac{e^{i \arccos x}-\Phi\left(a_{j}\right)}{1-\Phi\left(a_{j}\right) e^{i \arccos x}}\right)^{m_{j}}\right. \\
& \left.+\prod_{j=1}^{\ell}\left(\frac{e^{-i \arccos x}-\Phi\left(a_{j}\right)}{1-\Phi\left(a_{j}\right) e^{-i \arccos x}}\right)^{m_{j}}\right\} .
\end{aligned}
$$

В заключение сделаем общее замечание относительно используемого здесь метода, основанного на свойствах $\Psi$-функции - решении специальной краевой задачи Римана. Такая задача рассматривается при фиксированном $n \in \mathbb{N}\left(\right.$ см. задачи $\left(\mathcal{R}_{1}\right)$ и $\left.\left(\mathcal{R}_{2}\right)\right)$, a ее решение находится в явном виде в терминах римановой поверхности $\mathfrak{R}$ и веса $\rho$ (см. (16) и (21)). Отсюда следует, что этот метод пригоден и для нахождения формул сильной асимптотики в случае переменного (т.е. зависящего от $n$ ) веса $\rho=\rho_{n}$ : в явную формулу для $\Psi$-функции вместо веса $\rho_{n}$ надо подставить выражение, описывающее его зависимость от $n$ (см. [18]). Кроме того, метод оказывается применим и в случае, когда в (5) носитель $S$ меры $\sigma$ состоит из нескольких отрезков (подробнее см. ниже $\S 7$ ).

\section{§ 3. Доказательство предложения 1}

1. Пусть $\Psi$ - решение задачи $\left(\mathcal{R}_{1}\right)$ при некотором $n \in \mathbb{N}$, полином $Q_{n}(z)=Q_{n}(z ; \widehat{\rho}), \operatorname{deg} Q_{n} \leqslant n, Q_{n} \not \equiv 0$, удовлетворяет соотношению

$$
R_{n}(z ; \widehat{\rho}):=\left(Q_{n} \widehat{\rho}-P_{n}\right)(z)=O\left(\frac{1}{z^{n+1}}\right), \quad z \rightarrow \infty
$$

где $\operatorname{deg} P_{n} \leqslant n-1, \quad R_{n}(z)=R_{n}(z ; \widehat{\rho})-$ функция остатка. 
Определим на $\mathfrak{R} \backslash \Gamma$ функцию $F$ следующим образом:

$$
F(\mathbf{z})=\Psi(\mathbf{z}) \cdot \begin{cases}R_{n}(z) w(z), & \mathbf{z}=z^{(1)} \in D^{(1)} \\ -2 Q_{n}(z), & \mathbf{z}=z^{(2)} \in D^{(2)}\end{cases}
$$

нетрудно видеть, что $F$ (кусочно) голоморфна в $\mathfrak{R} \backslash \Gamma$.

Используя свойства $\Psi$-функции как решения задачи $\left(\mathcal{R}_{1}\right)$ и свойства функции остатка $R_{n}$ на границе отрезка $\Delta: R_{n}^{+}(x)-$ $R_{n}^{-}(x)=-2 Q_{n}(x) \rho(x) / w^{+}(x)$, получаем следующее краевое условие для $F$ на кривой $\Gamma$ :

$$
F(\boldsymbol{\zeta}+)-F(\boldsymbol{\zeta}-)=\chi(\boldsymbol{\zeta}-) \frac{1}{\rho(\zeta)}, \quad \boldsymbol{\zeta} \in \Gamma,
$$

где функция $\chi(\mathbf{z})=\Psi(\mathbf{z}) R_{n}(z) w(\mathbf{z}), \mathbf{z} \in D^{(2)}$, голоморфна на втором листе ${ }^{8}$. При этих условиях $F$ стандартным образом $[20, \S 8$, формула (8.16)] восстанавливается в $\mathfrak{R} \backslash \Gamma$ по граничным условиям (25) интегралом типа Коши:

$$
F(\mathbf{z})=-\frac{1}{2 \pi i} \int_{\Gamma^{+}} \chi(\boldsymbol{\zeta}-) \frac{1}{\rho(\zeta)} d \Omega(\boldsymbol{\zeta} ; \mathbf{z})+C_{n}, \quad \mathbf{z} \notin \Gamma,
$$

где $C_{n}$ - некоторая постоянная,

$$
d \Omega(\boldsymbol{\zeta} ; \mathbf{z})=\frac{1}{2} \frac{w(\boldsymbol{\zeta})+w(\mathbf{z})}{\zeta-z} \frac{d \zeta}{w(\boldsymbol{\zeta})}
$$

- мероморфный на $\Re$ абелев дифференциал, который имеет полюсы только в точках $\mathbf{z}, \infty^{(1)}, \infty^{(2)}$ с вычетами $1,-1 / 2,-1 / 2$ соответственно, а контур $\Gamma^{+}$ориентирован положительно относительно области $D^{(1)}$ : область остается слева при обходе по контуру. Интегральное представление (26) лежит в основе дальнейших рассуждений.

2. Пусть сначала $\mathbf{z}=z^{(1)} \in D^{(1)}$. Выберем числа $R>1$ и $\varepsilon>0$ так, чтобы функции $\chi$ и $1 / \rho$ были бы голоморфны в области $\left\{z^{(2)}: 1<|\Phi(z)|<R+\varepsilon\right\}$. Тогда (при $\mathbf{z}=z^{(1)}$ ) в интегральном представлении (26) контур Г можно заменить на контур

\footnotetext{
${ }^{8}$ Как и ранее, под $F(\boldsymbol{\zeta}+)$ здесь понимаются предельные значения функции $F(\mathbf{z})$ при $D^{(1)} \ni \mathbf{z} \rightarrow \boldsymbol{\zeta} \in \Gamma$. Аналогичный смысл придается $F(\boldsymbol{\zeta}-)$ и $\chi(\boldsymbol{\zeta}-)$.
} 
$\Gamma_{R}^{(2)}=\left\{z^{(2)}:|\Phi(z)|=R\right\}$, целиком лежащий на втором листе $D^{(2)}$. Воспользуемся теперь тем, что

$$
\chi\left(z^{(2)}\right)=\Psi\left(z^{(2)}\right) R_{n}(z) w\left(z^{(2)}\right)=-F\left(z^{(1)}\right) \frac{\Psi\left(z^{(2)}\right)}{\Psi\left(z^{(1)}\right)}=-\frac{F\left(z^{(1)}\right)}{\Psi\left(z^{(1)}\right)^{2}}
$$

и $d \Omega\left(\zeta^{(2)} ; z^{(1)}\right)=d \Omega\left(\zeta^{(1)} ; z^{(2)}\right)$ (см. (27)). Тогда из (26) получаем следующее представление для функции $F$ :

$$
\begin{aligned}
F\left(z^{(1)}\right) & =-\frac{1}{2 \pi i} \int_{\Gamma_{R}^{(2)}} \chi\left(\zeta^{(2)}\right) \frac{1}{\rho(\zeta)} d \Omega\left(\zeta^{(2)} ; z^{(1)}\right)+C_{n} \\
& =-\frac{1}{2 \pi i} \int_{\Gamma_{R}^{(1)}} \frac{F\left(\zeta^{(1)}\right)}{\Psi\left(\zeta^{(1)}\right)^{2} \rho(\zeta)} d \Omega\left(\zeta^{(1)} ; z^{(2)}\right)+C_{n}
\end{aligned}
$$

где $\Gamma_{R}^{(1)}=\left\{\zeta^{(1)}:|\Phi(\zeta)|=R\right\}$. При $\zeta^{(1)} \in \Gamma_{R}^{(1)}$ дифференциал $(27)$ имеет вид:

$$
d \Omega\left(\zeta^{(1)} ; z^{(2)}\right)=\frac{1}{2} \frac{w(\zeta)-w(z)}{\zeta-z} \frac{d \zeta}{w(\zeta)}
$$

следовательно, это - голоморфный по переменной $z^{(2)}$ в области $D^{(2)}$ дифференциал. Более того, дифференциал $d \Omega\left(\zeta^{(1)} ; \mathbf{z}\right)-$ голоморфный по $\mathbf{z}$ на компакте $D^{(2)} \sqcup \Gamma$ при $\zeta^{(1)} \in \Gamma_{R}^{(1)}$. Поэтому представление $(28)$ справедливо ${ }^{9}$ при $\mathbf{z} \in D^{(1)} \sqcup \Gamma$. Из (28) получаем, что равномерно на компакте $D^{(1)} \sqcup \Gamma$

$$
\left|F(\mathbf{z})-C_{n}\right|=M_{n} \max _{\Gamma_{R}^{(1)}} \frac{1}{\left|\Psi\left(\zeta^{(1)}\right)\right|^{2}} O(1) \quad \text { при } n \rightarrow \infty,
$$

где $M_{n}=\max _{\Gamma_{R}^{(1)}}\left|F\left(\zeta^{(1)}\right)\right|$. Следовательно, равномерно по $\mathbf{z} \in$ $D^{(1)} \sqcup \Gamma$

$$
\left|F(\mathbf{z})-C_{n}\right|=M_{n} \cdot O\left(\delta^{n}\right) \text { при } n \rightarrow \infty,
$$

где $\delta=1 / R^{2}<1$. Так как $F\left(z^{(1)}\right) \not \equiv 0$ (см. (24)), то $M_{n} \neq 0$, поэтому, применяя (30) сначала к компакту $\Gamma_{R}^{(1)} \subset D^{(1)}$, а затем

\footnotetext{
${ }^{9}$ Интегральная формула (28) задает аналитическое продолжение функции $F(\mathbf{z}), \mathbf{z} \in D^{(1)}$, через контур $\Gamma$ на второй лист римановой поверхности $\Re$ вплоть до контура $\Gamma_{R}^{(2)}$, причем для $\mathbf{z} \in D^{(2)}$ это продолжение, вообще говоря, не совпадает с функцией $F(\mathbf{z}), \mathbf{z} \in D^{(2)}$, определенной в (24); ср. с доказательством теоремы 2.1 в [14].
} 
к компакту $D^{(1)} \sqcup \Gamma$, последовательно получаем:

$$
\begin{aligned}
M_{n}=\left|C_{n}\right|\left(1+O\left(\delta^{n}\right)\right) & \text { при } n \rightarrow \infty, \\
F(\mathbf{z})=C_{n}\left(1+O\left(\delta^{n}\right)\right) & \text { при } n \rightarrow \infty \\
& \text { равномерно по } \mathbf{z} \in D^{(1)} \sqcup \Gamma .
\end{aligned}
$$

3. Пусть теперь $\mathbf{z}=z^{(2)} \in D^{(2)}$. Тогда из (26) вытекает, что при $z^{(2)} \in D_{R}^{(2)}=\left\{z^{(2)}:|\Phi(z)|>R\right\}$

$$
\begin{aligned}
F\left(z^{(2)}\right) & =-\frac{1}{2 \pi i} \int_{\Gamma_{R}^{(2)}} \chi\left(\zeta^{(2)}\right) \frac{1}{\rho(\zeta)} d \Omega\left(\zeta^{(2)} ; z^{(2)}\right)+C_{n} \\
& =-\frac{1}{2 \pi i} \int_{\Gamma_{R}^{(1)}} \frac{F\left(\zeta^{(1)}\right)}{\Psi\left(\zeta^{(1)}\right)^{2} \rho(\zeta)} d \Omega\left(\zeta^{(1)} ; z^{(1)}\right)+C_{n}
\end{aligned}
$$

здесь мы воспользовались тем, что $d \Omega\left(\zeta^{(2)} ; z^{(2)}\right)=d \Omega\left(\zeta^{(1)} ; z^{(1)}\right)$. Дифференциал $d \Omega\left(\zeta^{(1)} ; z^{(1)}\right)$ - голоморфный по $z^{(1)} \in D_{R}^{(1)}$ при $\zeta^{(1)} \in \Gamma_{R}^{(1)}$. Используя свойства $\Psi$-функции и полученное выше асимптотическое представление (31) для функции $F\left(z^{(1)}\right)$, получаем из $(32)$ аналог соотношения $(31)$ для $F\left(z^{(2)}\right)$ при $n \rightarrow \infty$ :

$$
F\left(z^{(2)}\right)=C_{n}\left(1+O\left(\delta_{1}^{n}\right)\right)
$$

равномерно внутри $D_{R}^{(2)}$, где $\delta_{1}<1$. Отсюда и из определения $(24)$ функции $F$ вытекает, что равномерно внутри $D_{R}$ справедливо представление

$$
\begin{gathered}
2 Q_{n}(z)=-\frac{C_{n}}{\Psi\left(z^{(2)}\right)}(1+o(1))=-C_{n} \Psi(z)(1+o(1)), \\
\text { где } \quad o(1)=O\left(\delta_{1}^{n}\right), \quad \delta_{1}<1 .
\end{gathered}
$$

Напомним, что

$$
\frac{\Psi(z)}{z^{n}} \rightarrow \varkappa_{n} \quad \text { при } \quad z \rightarrow \infty,
$$

где $\varkappa_{n}=2^{n} \exp \{S(\infty)\} \neq 0$, т.е. $\Psi$ имеет в точке $z=\infty$ полюс $n$-го порядка. Так как постоянная $C_{n} \neq 0$ при достаточно больших $n$ (см. (31)), то $\operatorname{deg} Q_{n}=n$, т.е. все достаточно далекие индексы $n$ нормальны. Выбор постоянной $C_{n} \neq 0$ связан только с нормировкой $Q_{n}$. Положим $C_{n}=-2$. В таком случае асимптотическая формула (34) для полиномов $Q_{n}$ вне отрезка $\Delta$ примет вид:

$$
Q_{n}(z)=\Psi(z)(1+o(1)), \quad n \rightarrow \infty,
$$

где $o(1)=O\left(\delta^{n}\right), \quad \delta<1$. 
4. Вернемся теперь к соотношению $F(\mathbf{z})=C_{n}(1+o(1))=$ $-2+o(1)$, которое справедливо равномерно на компакте $D^{(1)} \sqcup \Gamma$ и, следовательно, равномерно на Г. Из этого соотношения в силу определения функции $F$ вытекает, что равномерно по $x \in \Delta$

$$
\begin{aligned}
& \Psi^{+}(x) R_{n}^{+}(x) w^{+}(x)=-2+o(1), \quad n \rightarrow \infty . \\
& \Psi^{-}(x) R_{n}^{-}(x) w^{-}(x)=-2+o(1) \quad
\end{aligned}
$$

Так как $\Psi^{+}(x) \neq 0$ и $\Psi^{-}(x) \neq 0$ на $\Delta$, то

$$
\begin{aligned}
& R_{n}^{+}(x) w^{+}(x)=-\frac{2}{\Psi^{+}(x)}(1+o(1)), \quad n \rightarrow \infty . \\
& R_{n}^{-}(x) w^{-}(x)=-\frac{2}{\Psi^{-}(x)}(1+o(1)),
\end{aligned}
$$

Суммируя два последних соотношения, получаем

$$
R_{n}^{+}(x) w^{+}(x)+R_{n}^{-}(x) w^{-}(x)=-\frac{2}{\Psi^{+}(x)}-\frac{2}{\Psi^{-}(x)}+o(1) .
$$

Поскольку $R_{n}^{+}(x)-R_{n}^{-}(x)=-2 Q_{n}(x) \rho(x) / w^{+}(x)$ при $x \in \Delta$, то

$$
R_{n}^{+}(x) w^{+}(x)+R_{n}^{-}(x) w^{-}(x)=-2 Q_{n}(x) \rho(x) .
$$

Объединяя соотношения (36) и (37), получаем, что

$$
Q_{n}(x) \rho(x)=\frac{1}{\Psi^{+}(x)}+\frac{1}{\Psi^{-}(x)}+o(1) .
$$

Так как $\rho(x) \Psi^{+}(x) \Psi^{-}(x) \equiv 1$ при $x \in \Delta$, то

$$
Q_{n}(x)=\Psi^{+}(x)+\Psi^{-}(x)+o(1)
$$

равномерно по $x \in \Delta$, где $o(1)=o\left(\delta^{n}\right), \delta<1$.

Предложение 1 доказано.

\section{§ 4. Доказательство теоремы 2}

1. Схема доказательства теоремы 1, предложенная в [4] для положительной меры $\sigma$, является достаточно общей и при условии существования сильной асимптотики (35) позволяет получить аналог теоремы 1 для комплексного веса $\rho$. Ниже мы приводим доказательство теоремы 2 , следуя в целом схеме рассуждений работы [4]. 
В этом параграфе мы будем придерживаться следующего соглашения при обозначении полюсов функции $f$ в $D: a_{j}, j=1, \ldots, \ell$, - попарно различные полюсы $f$ кратностей $m_{j} \geqslant 1$ соответственно, $m=\sum_{j=1}^{\ell} m_{j}$ - полное число полюсов $f$ в $D$ с учетом их кратностей.

Пусть функция $f=\widehat{\rho}+r$, где

$$
\begin{aligned}
& \widehat{\rho}(z)=\frac{1}{\pi} \int_{\Delta} \frac{\rho(x)}{z-x} \frac{d x}{\sqrt{1-x^{2}}}, \\
& r(z)=\sum_{j=1}^{\ell} \sum_{k=1}^{m_{j}} \frac{A_{j, k}}{(k-1) !} \frac{1}{\left(z-a_{j}\right)^{k}},
\end{aligned}
$$

здесь $A_{j, m_{j}} \neq 0$.

Так как для функции $\widehat{\rho}$ все достаточно далекие индексы $n$ нормальны, то $\operatorname{deg} Q_{n}(z ; \widehat{\rho})=n$ при $n \geqslant n_{0}$. Временно в пределах этого параграфа нам удобно, сохранив обозначения, перейти $\kappa$ другой нормировке полиномов $Q_{n}(z ; \widehat{\rho})$ и считать, что $Q_{n}(z ; \widehat{\rho})=z^{n}+\cdots$ nри $n \geqslant n_{0}$. С учетом представления (16) функции $\Psi$ нетрудно видеть, что формула сильной асимптотики (35) для полиномов $Q_{n}$ сохранится, если мы соответствующим образом изменим и нормировку самой $\Psi$-функции, точнее, в правой части представления $(16) \Phi(z)$ заменим на $\Phi(z) / 2$ и умножим эту правую часть на $\exp \{-S(\infty)\}$ (и, тем самым, нормируем $\Psi$-функцию условием $\Psi(z) / z^{n} \rightarrow 1$ при $\left.z \rightarrow \infty\right)$.

Аналогичное соглашение в пределах этого параграфа будет действовать и относительно полиномов $Q_{n}(z ; f):$ если $\Lambda=\left\{n_{k}\right\}$ - последовательность нормальных индексов для функции $f$, то $Q_{n}(z ; f)=z^{n}+\cdots$ nри $n \in \Lambda$. Напомним, что последовательность $\Lambda=\left\{n_{k}\right\}$ разбивает натуральный ряд чисел на множества $\Lambda_{k}=\left\{n: n_{k} \leqslant n<n_{k+1}\right\}$ такие, что $f_{n}=f_{n_{k}}$ для $n \in \Lambda_{k}$. Для произвольного $n \in \mathbb{N}$ существует единственная пара полиномов $P_{n}, Q_{n}$ (степени $\leqslant n$ ) такая, что: а) справедливо соотношение (4), b) полиномы $P_{n}$ и $Q_{n}$ не имеют общих нулей и с) старший коэффициент полинома $Q_{n}$ равен 1 . Всюду в этом параграфе через $P_{n}=P_{n}(f)$ и $Q_{n}=Q_{n}(f)$ обозначаются полиномы, удовлетворяющие условиям а)-c); будем называть их числителем и знаменателем рациональной функции $f_{n}, n \in \mathbb{N}$.

Всюду в дальнейшем мы придерживаемся этих соглашений и, кроме того, для краткости полагаем: $L_{n}(z):=Q_{n}(z ; \widehat{\rho})=z^{n}+\cdots$ 
при $n \geqslant n_{0}, Q_{n}(z):=Q_{n}(z ; f)$; тем самым, $Q_{n}(z)=z^{n}+\cdots$ при $n \in \Lambda$.

С учетом сказанного из предложения 1 вытекают следующие формулы сильной асимптотики для полиномов $L_{n}$ при $n \rightarrow \infty$ :

$$
\begin{aligned}
L_{n}(z)= & \left(\frac{\Phi(z)}{2}\right)^{n} e^{S(z)-S(\infty)}(1+o(1)) \\
& \text { равномерно внутри } D \\
L_{n}(x)= & \left(\frac{\Phi^{+}(x)}{2}\right)^{n} e^{S^{+}(x)-S(\infty)}+\left(\frac{\Phi^{-}(x)}{2}\right)^{n} e^{S^{-}(x)-S(\infty)}+o(1) \\
& \text { равномерно на } \Delta .
\end{aligned}
$$

Отметим несколько фактов относительно полиномов $L_{n}$, ортогональных на отрезке $\Delta$ с весом $\rho$, которые вытекают из $(40),(41)$ и будут использоваться нами в дальнейшем. Пусть $U-$ произвольная окрестность отрезка $\Delta$ вида $U=U_{\varepsilon}=\left\{z: g_{D}(z, \infty)<\varepsilon\right\}$. Из формулы сильной асимптотики (40) для $L_{n}$ вытекает, что для достаточно больших $n$ все нули $L_{n}$ и их производных лежат внутри $U$. В дальнейшем будем считать, что $\varepsilon>0$ выбрано так, что все полюсы функции $f$ лежат в $D \backslash \bar{U}$.

Из формулы (41) легко вытекает, что $L_{n}(x)=2^{-n} \cdot O(1)$ равномерно на $\Delta$ и

$$
\int_{\Delta} L_{n}^{2}(x) \frac{\rho(x) d x}{\sqrt{1-x^{2}}}=2^{1-2 n} e^{-2 S(\infty)}(1+o(1)) \quad \text { при } \quad n \rightarrow \infty
$$

(cp. с (19)).

Исходя из этих свойств полиномов $L_{n}$ легко доказать следующие утверждения (аналоги лемм 2-5 из [4]).

(i) Равномерно внутри D справедливо соотношение

$$
\lim _{n \rightarrow \infty} \frac{L_{n+\mu}}{L_{n}}(z)=\left(\frac{\Phi(z)}{2}\right)^{\mu}
$$

где $\mu$ - произвольное натуралъное число.

(ii) Для любых натуральных $\mu u \nu, \mu<\nu<n, u$ любого $K \Subset$ $\mathbb{C} \backslash \bar{U}$ имеют место оценки

$$
c_{1} n^{\nu-\mu}<\left|\frac{L_{n}^{(\nu)}}{L_{n}^{(\mu)}}(z)\right|<c_{2} n^{\nu-\mu}, \quad z \in K
$$

где $c_{1}>0$ и $c_{2}<+\infty$ не зависят от $n$. 
(iii) Для любых натуралъных $\mu$ и равномерно внутри $D$ имеe.M:

$$
\lim _{n \rightarrow \infty} \frac{L_{n+\mu}^{(\nu)}}{L_{n}^{(\nu)}}(z)=\left(\frac{\Phi(z)}{2}\right)^{\mu}
$$

(iv) Для любых иельх неотрицательных $\mu$ и и любого $a \in D$ имеем:

$$
\lim _{n \rightarrow \infty}\left(\frac{\left\|L_{n+\mu}\right\|_{\Delta}}{\left|L_{n}^{(\nu)}(a)\right|}\right)^{1 / n}=\frac{1}{|\Phi(a)|}
$$

где $\|\cdot\|_{\Delta}-\operatorname{sup-норма~на~} \Delta$.

Из определяющего рациональную дробь $f_{n}$ соотношения (4) для $f=\widehat{\rho}+r$ легко вытекает, что для любого полинома $q, \operatorname{deg} q \leqslant$ $n-1$, имеем

$$
\int_{\Delta} Q_{n}(x) q(x) \frac{\rho(x) d x}{\sqrt{1-x^{2}}}+\left.\sum_{j=1}^{\ell} \sum_{k=1}^{m_{j}} A_{j, k}\left(Q_{n}(z) q(z)\right)^{(k-1)}\right|_{z=a_{j}}=0
$$

(напомним, что $\left.Q_{n}(z):=Q_{n}(z ; f)\right)$. Всюду в дальнейшем считаем, что $n>m$. Полагая $\omega_{m}(z)=\prod_{j=1}^{\ell}\left(z-a_{j}\right)^{m_{j}}, q(z)=\omega_{m}(z) z^{\nu}$, получим из (46) следующие соотношения "квазиортогональности" для полинома $\left(Q_{n} \omega_{m}\right)(z), \operatorname{deg}\left(Q_{n} \omega_{m}\right) \leqslant n+m$ :

$$
\int_{\Delta} Q_{n}(x) \omega_{m}(z) z^{\nu} \frac{\rho(x) d x}{\sqrt{1-x^{2}}}=0, \quad \nu=0,1, \ldots, n-m-1
$$

Так как все достаточно далекие индексы $n$ для функции $\widehat{\rho}$ нормальны, то $\operatorname{deg} L_{n}=n$ при $n \geqslant n_{0}$. Отсюда, из ортогональности $L_{n}$ с весом $\rho$ на $\Delta$ и соотношений квазиортогональности $(47)$ для $Q_{n}$ вытекает, что $\operatorname{deg} Q_{n} \geqslant n-2 m$ при всех $n \geqslant n_{0}+m$. Это означает, что для последовательности $\Lambda=\left\{n_{k}\right\}$ нормальных индексов для функции $f$ имеем:

$$
n_{k+1}-n_{k} \leqslant 2 m+1 \quad \text { при } n_{k} \geqslant n_{0}+m \text {. }
$$

Дальнейшие рассуждения (в пп. 2-4) проводятся для нормальных индексов. 
2. Наша ближайшая цель - доказательство следующего утверждения (формулы сравнительной асимптотики), аналогичного лемме 1 из [4].

ПРЕДЛОЖЕНИЕ 3. Равномерно внутри области D справедливо соотношение

$$
\lim _{\substack{n \rightarrow \infty \\ n \in \Lambda}} \frac{Q_{n}}{L_{n}}(z)=\frac{\prod_{j=1}^{\ell}\left(\Phi(z)-\Phi\left(a_{j}\right)\right)^{2 m_{j}}}{2^{m} \Phi^{m}(z) \prod_{j=1}^{\ell}\left(z-a_{j}\right)^{m_{j}}} .
$$

Пусть $n \in \Lambda, S_{n+m}(z)=\left(Q_{n} \omega_{m}\right)(z)=z^{n+m}+\cdots$. Тогда

$$
\int_{\Delta} S_{n+m}(x) x^{\nu} \frac{\rho(x) d x}{\sqrt{1-x^{2}}}=0, \quad \nu=0,1, \ldots, n-m-1 .
$$

Так как для всех достаточно далеких индексов $\operatorname{deg} L_{n}=n$, то из этих соотношений вытекает, что полином $S_{n+m}$ представляется в следующем виде:

$$
S_{n+m}=L_{n+m}+\lambda_{n, 1} L_{n+m-1}+\cdots+\lambda_{n, 2 m} L_{n-m},
$$

где параметры $\lambda_{1, n}, \ldots, \lambda_{n, 2 m}$ неизвестны. Ниже мы получим $2 m$ соотношений, которые позволят исключить эти параметры из представления (50).

Первые $m$ соотношений немедленно следуют из определения полинома $S_{n+m}$ :

$$
S_{n+m}\left(a_{j}\right)=S_{n+m}^{\prime}\left(a_{j}\right)=\cdots=S_{n+m}^{\left(m_{j}-1\right)}\left(a_{j}\right)=0, \quad j=1, \ldots, \ell
$$

Получим еще одну группу из $m$ соотношений.

Зафиксируем $j \in\{1, \ldots, \ell\}$ и положим

$$
S_{n+m}(z) \cdot\left(z-a_{j}\right)^{-m_{j}}=S_{n+m, j}(z)=b_{n, j}^{(0)}+b_{n, j}^{(1)}\left(z-a_{j}\right)+\cdots
$$

(степень этого полинома равна $n+m-m_{j}$ ). Подставив теперь полином $\omega_{m}(z) /\left(z-a_{j}\right)^{\nu}, \nu=1, \ldots, m_{j}$, в соотношение $(46)$, получим

$$
\int_{\Delta} \frac{S_{n+m}(x)}{\left(x-a_{j}\right)^{\nu}} \frac{\rho(x) d x}{\sqrt{1-x^{2}}}+\left.\sum_{k=1}^{m_{j}} A_{j, k}\left(S_{n+m, j}(z)\left(z-a_{j}\right)^{m_{j}-\nu}\right)^{(k-1)}\right|_{z=a_{j}}=0 .
$$


Отсюда вытекает система уравнений

$$
\begin{aligned}
& \int_{\Delta} \frac{S_{n+m}(x)}{x-a_{j}} \frac{\rho(x) d x}{\sqrt{1-x^{2}}}+\left(m_{j}-1\right) ! A_{j, m_{j}} b_{n, j}^{(0)}=0, \\
& \int_{\Delta} \frac{S_{n+m}(x)}{\left(x-a_{j}\right)^{2}} \frac{\rho(x) d x}{\sqrt{1-x^{2}}}+\left(m_{j}-2\right) ! A_{j, m_{j}-1} b_{n, j}^{(0)} \\
& \quad+\left(m_{j}-1\right) ! A_{j, m_{j}} b_{n, j}^{(1)}=0, \\
& \ldots \ldots \ldots \ldots \ldots \ldots \ldots \ldots \ldots \ldots \ldots \ldots \ldots \\
& \int_{\Delta} \frac{S_{n+m}(x)}{\left(x-a_{j}\right)^{m_{j}}} \frac{\rho(x) d x}{\sqrt{1-x^{2}}}+A_{j, 1} b_{n, j}^{(0)}+\ldots \ldots \\
& \quad+\left(m_{j}-1\right) ! A_{j, m_{j}} b_{n, j}^{\left(m_{j}-1\right)}=0 .
\end{aligned}
$$

Решая эту систему относительно $b_{n, j}^{(0)}, b_{n, j}^{(1)}, \ldots, b_{n, j}^{\left(m_{j}-1\right)}$ (напомним, что величины $A_{j, m_{j}} \neq 0$, см. $\left.(39)\right)$, получим набор из $m_{j}$ полиномов $p_{j, 1}, \ldots, p_{j, m_{j}}$ (второй индекс равен степени полинома) таких, что

$$
\begin{aligned}
& m_{j} ! b_{n, j}^{(0)}+\int_{\Delta} S_{n+m}(x) p_{j, 1}\left(\frac{1}{x-a_{j}}\right) \frac{\rho(x) d x}{\sqrt{1-x^{2}}}=0, \\
& \left(m_{j}+1\right) ! b_{n, j}^{(1)}+\int_{\Delta} S_{n+m}(x) p_{j, 2}\left(\frac{1}{x-a_{j}}\right) \frac{\rho(x) d x}{\sqrt{1-x^{2}}}=0, \\
& \ldots \ldots \ldots \ldots \ldots \ldots \ldots \ldots \ldots \ldots \ldots \ldots \ldots \ldots \ldots \ldots \ldots \ldots \ldots \ldots \ldots \ldots \ldots \ldots \ldots \ldots \ldots \ldots \\
& \left(2 m_{j}-1\right) ! b_{n, j}^{\left(m_{j}-1\right)}+\int_{\Delta} S_{n+m}(x) p_{j, m_{j}}\left(\frac{1}{x-a_{j}}\right) \frac{\rho(x) d x}{\sqrt{1-x^{2}}}=0 .
\end{aligned}
$$

Подчеркнем, что полиномы $p_{j, \nu}, 1 \leqslant \nu \leqslant m_{j}$, не зависят от $n$; они полностью определяются главной частью разложения функции $f$ в точке $z=a_{j}$. Учитывая (52), последнюю систему равенств можно переписать так:

$$
\begin{aligned}
& S_{n+m}^{\left(m_{j}\right)}\left(a_{j}\right)+\int_{\Delta} S_{n+m}(x) p_{j, 1}\left(\frac{1}{x-a_{j}}\right) \frac{\rho(x) d x}{\sqrt{1-x^{2}}}=0, \\
& S_{n+m}^{\left(m_{j}+1\right)}\left(a_{j}\right)+\int_{\Delta} S_{n+m}(x) p_{j, 2}\left(\frac{1}{x-a_{j}}\right) \frac{\rho(x) d x}{\sqrt{1-x^{2}}}=0, \\
& \ldots \ldots \ldots \ldots \ldots \ldots \ldots \ldots \ldots \ldots \ldots \ldots \ldots \ldots \ldots \ldots \ldots \ldots \ldots \ldots \ldots \ldots \ldots \ldots \\
& S_{n+m}^{\left(2 m_{j}-1\right)}\left(a_{j}\right)+\int_{\Delta} S_{n+m}(x) p_{j, m_{j}}\left(\frac{1}{x-a_{j}}\right) \frac{\rho(x) d x}{\sqrt{1-x^{2}}}=0 .
\end{aligned}
$$

Совокупность таких систем (53) для всех $j=1,2, \ldots, \ell$ содержит $m$ соотношений. 


\section{3. Положим}

$$
\delta_{n}(j, \mu, \nu)=\frac{1}{L_{n-m}^{\left(m_{j}-1+\nu\right)}\left(a_{j}\right)} \int_{\Delta} L_{n-m+\mu}(x) p_{j, \nu}\left(\frac{1}{x-a_{j}}\right) \frac{\rho(x) d x}{\sqrt{1-x^{2}}},
$$

где $j=1, \ldots, \ell, \nu=1, \ldots, m_{j}, \quad \mu=0,1, \ldots, 2 m$. Функции

$$
p_{j, \nu}\left(\frac{1}{z-a_{j}}\right)
$$

голоморфны в окрестности отрезка $\Delta$, а полиномы $p_{j, \nu}$ не зависят от $n$. С учетом свойств (i)-(iv) полиномов $L_{n}$, получаем из (54):

$$
\varlimsup_{n \rightarrow \infty}\left|\delta_{n}(j, \mu, \nu)\right|^{1 / n} \leqslant \frac{1}{\left|\Phi\left(a_{j}\right)\right|}<1 .
$$

Пусть $\delta<1$ таково, что $1 /\left|\Phi\left(a_{j}\right)\right|<\delta<1$ при каждом $j=1, \ldots, \ell$. Тогда для любой тройки индексов $j, \mu, \nu$, меняющихся в указанных выше пределах, имеем

$$
\delta_{n}(j, \mu, \nu)=o\left(\delta^{n}\right), \quad n \rightarrow \infty .
$$

Подставим в соотношения (53) разложение (50); после очевидных преобразований, учитывая (55), получим

$$
\begin{aligned}
& \frac{L_{n+m}}{L_{n-m}}\left(a_{j}\right)+\lambda_{n, 1} \frac{L_{n+m-1}}{L_{n-m}}\left(a_{j}\right)+\cdots+\lambda_{n, 2 m}=0, \\
& \ldots \ldots \ldots \ldots \ldots \ldots \ldots \ldots \ldots \ldots \ldots \ldots \ldots \ldots \ldots \ldots \ldots \ldots \ldots \ldots \ldots \ldots \\
& \frac{L_{n+m}^{\left(m_{j}-1\right)}}{L_{n-m}^{\left(m_{j}-1\right)}}\left(a_{j}\right)+\lambda_{n, 1} \frac{L_{n+m-1}^{\left(m_{j}-1\right)}}{L_{n-m}^{\left(m_{j}-1\right)}}\left(a_{j}\right)+\cdots+\lambda_{n, 2 m}=0, \\
& \frac{L_{n+m}^{\left(m_{j}\right)}}{L_{n-m}^{\left(m_{j}\right)}}\left(a_{j}\right)+o\left(\delta^{n}\right)+\lambda_{n, 1}\left(\frac{L_{n+m-1}^{\left(m_{j}\right)}}{L_{n-m}^{\left(m_{j}\right)}}\left(a_{j}\right)+o\left(\delta^{n}\right)\right)+\cdots \\
& \quad+\lambda_{n, 2 m}\left(1+o\left(\delta^{n}\right)\right)=0, \\
& \quad \ldots \ldots \ldots \ldots \ldots \ldots \ldots \ldots \\
& \frac{L_{n+m}^{\left(2 m_{j}-1\right)}}{L_{n-m}^{\left(2 m_{j}-1\right)}}\left(a_{j}\right)+o\left(\delta^{n}\right)+\lambda_{n, 1}\left(\frac{L_{n+m-1}^{\left(2 m_{j}-1\right)}}{L_{n-m}^{\left(2 m_{j}-1\right)}}\left(a_{j}\right)+o\left(\delta^{n}\right)\right)+\cdots \\
& \quad+\lambda_{n, 2 m}\left(1+o\left(\delta^{n}\right)\right)=0,
\end{aligned}
$$


где $j=1,2, \ldots, \ell$. Во второй группе из $m_{j}$ соотношений мы заменили на $o\left(\delta^{n}\right)$ выражения вида (54).

При $n \rightarrow \infty$ в каждом из уравнений (56) строка коэффициентов имеет один и тот же предел, а именно строку

$$
\left(\frac{1}{2} \Phi\left(a_{j}\right)\right)^{2 m},\left(\frac{1}{2} \Phi\left(a_{j}\right)\right)^{2 m-1}, \ldots, 1
$$

(см. свойства (i)-(iv)). Преобразуем теперь систему (56) так, чтобы в пределе получались линейно независимые строки.

С помощью формулы Лейбница получаем

$$
\frac{L_{n+m-\mu}^{(\nu)}}{L_{n-m}^{(\nu)}}=\left(\frac{L_{n+m-\mu}}{L_{n-m}}\right)^{(\nu)} \frac{L_{n-m}}{L_{n-m}^{(\nu)}}-\sum_{k=1}^{\nu} F_{n-m}(\nu, k) \frac{L_{n+m-\mu}^{(\nu-k)}}{L_{n-m}^{(\nu-k)}},
$$

где

$$
F_{n-m}(\nu, k)=\left(\begin{array}{l}
\nu \\
k
\end{array}\right) \frac{L_{n-m} L_{n-m}^{(\nu-k)}}{L_{n-m}^{(\nu)}}\left(\frac{1}{L_{n-m}}\right)^{(k)},
$$

$\mu=0,1, \ldots, 2 m-1$; подчеркнем, что $F_{n-m}(\nu, k)$ не зависит от $\mu$. Пользуясь соотношениями (43) и (44), нетрудно показать, что для любого $a \in D$ справедливо неравенство

$$
\varlimsup_{n \rightarrow \infty}\left|F_{n-m}(\nu, k)(a)\right|^{1 / n} \leqslant 1 .
$$

Пусть $C>1$ таково, что $\delta_{1}=C \delta<1$. В силу (58) для любой тройки индексов $j, \nu, k$, меняющихся в пределах $j=1, \ldots, \ell$, $\nu=1, \ldots, 2 m_{j}-1, k=1, \ldots, \nu$, имеем

$$
F_{n-m}(\nu, k)\left(a_{j}\right)=O\left(C^{n}\right), \quad n \rightarrow \infty .
$$

Введем следующие обозначения:

$$
\varphi_{n, \mu}=\frac{L_{n+m-\mu}}{L_{n-m}}, \quad \mu=0,1, \ldots, 2 m ; \quad \varphi=\frac{\Phi}{2} .
$$

В силу (42) равномерно внутри $D$ выполняются соотношения:

$$
\lim _{n \rightarrow \infty} \varphi_{n, \mu}(z)=\varphi^{2 m-\mu}(z), \quad \mu=0,1, \ldots, 2 m ;
$$

тем самым, и последовательные производные функций $\varphi_{n, \mu}$ равномерно внутри $D$ сходятся к соответствующим производным функций $\varphi^{2 m-\mu}$. 
Прибавим теперь к $\nu$-й строке системы (56) линейную комбинацию предшествующих строк с коэффициентами, определяемыми равенством $(57)$ в точке $a_{j}$. Тогда, учитывая (для $\nu \geqslant m_{j}$ ) соотношение (59), получаем следующую систему:

$$
\begin{gathered}
\varphi_{n, 0}^{(\nu)}\left(a_{j}\right)+\lambda_{n, 1} \varphi_{n, 1}^{(\nu)}\left(a_{j}\right)+\cdots+\lambda_{n, 2 m} \varphi_{n, 2 m}^{(\nu)}\left(a_{j}\right)=0 \\
\nu=0,1, \ldots, m_{j}-1 ; \\
\varphi_{n, 0}^{(\nu)}\left(a_{j}\right) \frac{L_{n-m}}{L_{n-m}^{(\nu)}}\left(a_{j}\right)+o\left(\delta_{1}^{n}\right) \\
+\lambda_{n, 1}\left(\varphi_{n, 1}^{(\nu)}\left(a_{j}\right) \frac{L_{n-m}}{L_{n-m}^{(\nu)}}\left(a_{j}\right)+o\left(\delta_{1}^{n}\right)\right)+\cdots \\
+\lambda_{n, 2 m}\left(\varphi_{n, 2 m}^{(\nu)}\left(a_{j}\right) \frac{L_{n-m}}{L_{n-m}^{(\nu)}}\left(a_{j}\right)+o\left(\delta_{1}^{n}\right)\right)=0, \\
\nu=m_{j}, m_{j}+1, \ldots, 2 m_{j}-1 .
\end{gathered}
$$

Умножая $\nu$-е уравнение (для $\left.\nu \geqslant m_{j}\right)$ на $L_{n-m}^{(\nu)}\left(a_{j}\right) / L_{n-m}\left(a_{j}\right)$ и учитывая соотношения (43) (т.е. степенной рост этого множителя при $\left.\nu=m_{j}, \ldots, 2 m_{j}-1\right)$, окончательно получаем систему

$$
\begin{aligned}
& \varphi_{n, 0}\left(a_{j}\right)+\lambda_{n, 1} \varphi_{n, 1}\left(a_{j}\right)+\cdots+\lambda_{n, 2 m} \cdot 1=0, \\
& \varphi_{n, 0}^{\left(m_{j}-1\right)}\left(a_{j}\right)+\lambda_{n, 1} \varphi_{n, 1}^{\left(m_{j}-1\right)}\left(a_{j}\right)+\cdots+\lambda_{n, 2 m} \cdot 0=0, \\
& \varphi_{n, 0}^{\left(m_{j}\right)}\left(a_{j}\right)+o(1)+\lambda_{n, 1}\left(\varphi_{n, 1}^{\left(m_{j}\right)}\left(a_{j}\right)+o(1)\right)+\cdots \\
& +\lambda_{n, 2 m} \cdot o(1)=0, \\
& \varphi_{n, 0}^{\left(2 m_{j}-1\right)}\left(a_{j}\right)+o(1)+\lambda_{n, 1}\left(\varphi_{n, 1}^{\left(2 m_{j}-1\right)}\left(a_{j}\right)+o(1)\right)+\cdots \\
& +\lambda_{n, 2 m} \cdot o(1)=0 \text {, }
\end{aligned}
$$

где $j=1,2, \ldots, \ell$.

4. Обозначим через $\Delta_{n}^{0}$ определитель при неизвестных $\lambda_{n, 1}$, $\ldots, \lambda_{n, 2 m}$ этой системы. Учитывая соотношение $(61)$, получим

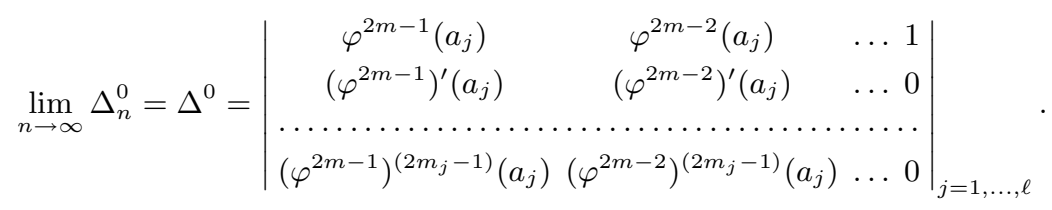


Символ, стоящий в правой части этого равенства, обозначает определитель порядка $2 m$, в котором последовательно выписываются указанные группы строк для $j=1,2, \ldots, \ell$. Поскольку $\varphi-$ однолистная функция в области $D$, определитель $\Delta^{0}$ отличен от нуля (величина этого определителя нам не понадобится). Следовательно, $\Delta_{n}^{0} \neq 0$ при достаточно больших $n \geqslant n_{0}$.

Добавим теперь к системе (62) соотношение

$$
\varphi_{n, 0}(z)+\lambda_{n, 1} \varphi_{n, 1}(z)+\cdots+\lambda_{n, 2 m}=\frac{S_{n+m}}{L_{n-m}}(z), \quad z \in D,
$$

и исключим из этого соотношения (с помощью системы (62)) неизвестные $\lambda_{n, 1}, \ldots, \lambda_{n, 2 m}$. Получим

$$
\frac{S_{n+m}}{L_{n-m}}(z)=\frac{\Delta_{n}(z)}{\Delta_{n}^{0}}, \quad z \in D, \quad n \geqslant n_{0},
$$

где

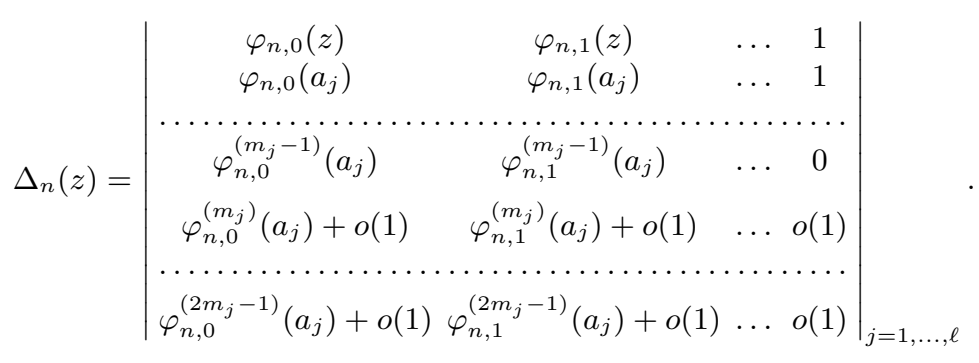

Следовательно (напомним, что $n \in \Lambda$ ),

$$
\lim _{\substack{n \rightarrow \infty \\ n \in \Lambda}} \frac{S_{n+m}}{L_{n-m}}(z)=\mathcal{P}(z)=\frac{\Delta(z)}{\Delta^{0}}, \quad z \in D
$$

где

$$
\Delta(z)=\left|\begin{array}{cccc}
\varphi^{2 m}(z) & \varphi^{2 m-1}(z) & \ldots & 1 \\
\varphi^{2 m}\left(a_{1}\right) & \ldots \ldots \ldots \ldots \ldots & \ldots \\
\vdots & \vdots & \Delta^{0} \\
\left(\varphi^{2 m}\right)^{\left(2 m_{\ell}-1\right)}\left(a_{\ell}\right) & \vdots &
\end{array}\right|
$$

переход к пределу в (63) - равномерный внутри $D$. Функция $\mathcal{P}(z)$ - полином от $\varphi(z)$ степени $2 m$ со старшим коэффициентом 
при $\varphi^{2 m}(z)$, равным 1 (см. (50)). Учитывая характер нулей этой функции, получаем

$$
\mathcal{P}(z)=\prod_{j=1}^{\ell}\left(\varphi(z)-\varphi\left(a_{j}\right)\right)^{2 m_{j}}
$$

Следовательно, принимая во внимание (60), (65) и введенное ранее обозначение $S_{n+m}=Q_{n} \omega_{m}$, имеем:

$$
\lim _{\substack{n \rightarrow \infty \\ n \in \Lambda}} \frac{Q_{n}}{L_{n-m}}(z)=\frac{\prod_{j=1}^{\ell}\left(\Phi(z)-\Phi\left(a_{j}\right)\right)^{2 m_{j}}}{2^{2 m} \omega_{m}(z)}, \quad z \in D ;
$$

сходимость - равномерная внутри $D$. Последнее соотношение эквивалентно (49). Предложение 3 доказано.

Отметим, что в силу легко проверяемого тождества

$$
\frac{\Phi(z)-\Phi(a)}{z-a}=-\frac{2 \Phi(z) \Phi(a)}{1-\Phi(z) \Phi(a)}
$$

соотношение (49) эквивалентным образом переписывается в виде:

$$
\lim _{\substack{n \rightarrow \infty \\ n \in \Lambda}} \frac{Q_{n}(z)}{L_{n}(z)}=\mathrm{const} \cdot \prod_{j=1}^{\ell}\left(\frac{\Phi(z)-\Phi\left(a_{j}\right)}{1-\Phi(z) \Phi\left(a_{j}\right)}\right)^{m_{j}}
$$

где const $=(-1)^{m} / \prod_{j=1}^{\ell} \Phi^{m_{j}}\left(a_{j}\right)$.

5. Функция, стоящая в правой части соотношения (49), голоморфна в области $D$; множество ее нулей совпадает (с учетом их кратностей) с множеством полюсов функции $f$ в $D$. Отсюда и из теоремы Руше получаем утверждение теоремы 2 для последовательности нормальных индексов $\Lambda=\left\{n_{k}\right\}$; структура последовательности $\left\{f_{n}\right\}_{n \in \mathbb{N}}$ такова (см. $\S 1$, п. 2), что тем самым это утверждение оказывается справедливым и для всех индексов.

Ниже мы используем следующее соотношение, вытекающее из (63):

$$
\lim _{\substack{n \rightarrow \infty \\ n \in \Lambda}}\left|S_{n+m}(z)\right|^{1 / n}=\frac{1}{2}|\Phi(z)|, \quad z \in D^{\prime}
$$

(переход к пределу - равномерный внутри $D^{\prime}$ ). 
Докажем утверждение о скорости сходимости функций $f_{n}$ к $f$ и полюсов $f_{n}$ к полюсам $f$. Пусть $n \in \Lambda, n>m$; из (4) получаем

$$
\left(\widehat{\rho}+r-f_{n}\right)(z)=\left(\widehat{\rho}-\tau_{n}\right)(z)=\frac{A}{z^{2 n+1}}+\cdots ;
$$

здесь $\tau_{n}=f_{n}-r$ - рациональная функция порядка $n+m$, знаменатель которой есть $Q_{n} \omega_{m}=S_{n+m}$. Соотношение (69) означает, что эта рациональная функция интерполирует голоморфную в области $D$ функцию $\widehat{\rho}$ в точке $z=\infty$ до порядка $2 n+1>n+m$.

Фиксируем произвольный компакт $K \subset D^{\prime}$; из утверждения теоремы 2 следует, что для достаточно больших $n$ все нули полинома $S_{n+m}$ лежат вне компакта $K$. Для любого $R>1$ положим $\Gamma_{R}=\{z:|\Phi(z)|=R\} ;$ пусть $R$ столь близко к 1 , что $K$ лежит во внешности $\Gamma_{R}$. С помощью формулы Эрмита из (69) получаем

$$
\left(f-f_{n}\right)(z)=\left(\widehat{\rho}-\tau_{n}\right)(z)=\frac{1}{2 \pi i} \int_{\Gamma_{R}} \frac{S_{n+m}(t) q(t) \widehat{\rho}(t) d t}{S_{n+m}(z) q(z)(t-z)}, \quad z \in K,
$$

где $q$ - произвольный полином степени $\leqslant n-m$. Выберем $q=$ $L_{n-m}$. Тогда, используя (68) и свойства полиномов $\left\{L_{n}\right\}$, получаем оценку

$$
\left\|f-f_{n}\right\|_{K}<C\left(\frac{R+\varepsilon}{R(K)-\varepsilon}\right)^{2 n},
$$

где $\|\cdot\|$ - sup-норма, $R(K)=\inf \{|\Phi(z)|: z \in K\}, \quad \varepsilon>0$ - произвольно, $n>n(\varepsilon), n \in \Lambda, C$ не зависит от $n$. Следовательно,

$$
\varlimsup_{\substack{n \rightarrow \infty \\ n \in \Lambda}}\left\|f-f_{n}\right\|_{K}^{1 / 2 n} \leqslant \frac{R+\varepsilon}{R(K)-\varepsilon} ;
$$

устремляя $\varepsilon$ к нулю и $R$ к единице, получаем отсюда

$$
\varlimsup_{\substack{n \rightarrow \infty \\ n \in \Lambda}}\left\|f-f_{n}\right\|_{K}^{1 / 2 n} \leqslant \frac{1}{R(K)}<1 .
$$

Вновь учитывая структуру последовательности $\left\{f_{n}\right\}_{n \in \mathbb{N}}$ и неравенство (48) для нормальных индексов, получаем из (70) следующее утверждение о скорости сходимости функций $f_{n}$ к $f$ :

$$
\varlimsup_{n \rightarrow \infty}\left\|f-f_{n}\right\|_{K}^{1 / 2 n} \leqslant \frac{1}{R(K)}<1 .
$$


Отсюда уже легко вытекает, что если $a \in D-$ полюс $f$ кратности $\mu \geqslant 1$, то

$$
\varlimsup_{n \rightarrow \infty}\left|a-\zeta_{j, n}(a)\right|^{1 / n} \leqslant \frac{1}{|\Phi(a)|^{2 / \mu}}<1, \quad j=1, \ldots, \mu
$$

(как обычно, $\zeta_{j, n}(a), j=1, \ldots, \mu,-$ полюсы $f_{n}$, перенумерованные в порядке неубывания их расстояния до точки $a)$.

\section{§5. Доказательство предложения 2}

1. Существование и единственность решения задачи $\left(\mathcal{R}_{2}\right)$ доказываются стандартным способом (см. [16]): в предположении, что решение существует, находится его явный вид. Непосредственно проверяется, что полученная в итоге функция является решением краевой задачи. Для доказательства предложения 2 нам понадобится рассмотреть и решить несколько более общую, чем $\left(\mathcal{R}_{2}\right)$, краевую задачу.

Пусть $a_{j}, b_{j} \in \mathbb{C} \backslash \Delta, j=1, \ldots, m,-$ произвольные необязательно различные точки.

ЗАДАчА $\left(\mathcal{R}_{3}\right)$. При биксированном $n \in \mathbb{N}$ найти функиию $\psi=\psi_{n}$ такую, что:

$1^{\circ}$. $\psi\left(\right.$ кусочно) мероморфна на $\mathfrak{R} \backslash \Gamma=D^{(1)} \sqcup D^{(2)}$;

$2^{\circ}$. дивизор $(\psi)=n \infty^{(2)}+a_{1}^{(1)}+\cdots+a_{m}^{(1)}-b_{1}^{(2)}-\cdots-b_{m}^{(2)}-n \infty^{(1)}$;

$3^{\circ}$. на Г выполнено краевое условие: $\rho(x) \psi(\mathbf{x}+)=\psi(\mathbf{x}-)$.

Непосредственно из условий этой задачи вытекает, что

$$
\psi\left(z^{(1)}\right) \psi\left(z^{(2)}\right) \equiv \mathrm{const} \cdot \frac{\prod_{j=1}^{m}\left(z-a_{j}\right)}{\prod_{j=1}^{m}\left(z-b_{j}\right)}
$$

при $z \in D$, где const $\neq 0$. Как и ранее, будем придерживаться той нормировки $\psi$-функции, которая даст const $=1$. Решение задачи $\left(\mathcal{R}_{3}\right)$ понадобится нам в ситуации, когда $b_{j}=a_{j, n} \rightarrow a_{j}$ при $n \rightarrow \infty, j=1, \ldots, m$. Отметим, что среди точек $a_{1}, \ldots, a_{m}$ в п. $2^{\circ}$ задачи $\left(\mathcal{R}_{3}\right)$ могут встречаться совпадающие; аналогичное замечание относится и к точкам $b_{1}, \ldots, b_{m}$. В дальнейшем в пределах этого параграфа подобного соглашения нам будет удобно придерживаться и относительно полюсов функции $f:$ каждый полюс $f$ в $D$ въписъвается столько раз, какова его кратность. 
Найти явный вид решения задачи $\left(\mathcal{R}_{3}\right)$ не составляет труда: риманова поверхность $\mathfrak{R}$ - нулевого рода, поэтому любой дивизор вида $a^{(1)}-b^{(2)}$ разрешим: существует функция $\Phi_{a, b}$, мероморфная на $\mathfrak{R}$ и такая, что $\left(\Phi_{a, b}\right)=a^{(1)}-b^{(2)}$ (см. (15)). Для $\psi$-функции $\operatorname{deg}(\psi)=0$, поэтому задача $\left(\mathcal{R}_{3}\right)$ разбивается на несколько независимых задач, соответствующих пп. $2^{\circ}$ и $3^{\circ}$.

Функция $\Phi(\mathbf{z})=z+w$ мероморфна на $\mathfrak{R}$, дивизор $(\Phi)=$ $\infty^{(2)}-\infty^{(1)}$ и $\Phi\left(z^{(1)}\right) \Phi\left(z^{(2)}\right) \equiv 1$ при $z \in D$. Пусть $a, b \in \mathbb{C} \backslash \Delta$ - произвольные точки, $\lambda \neq 0$ - комплексная постоянная. Функция

$$
\Phi_{a, b}(\mathbf{z})=\lambda \frac{\Phi(\mathbf{z})-\Phi(b)}{z-b} \frac{z-a}{1-\Phi(a) \Phi(\mathbf{z})}
$$

- однозначная мероморфная функция на $\mathfrak{R}$ с дивизором $\left(\Phi_{a, b}\right)=$ $a^{(1)}-b^{(2)}$. Положим $\lambda=\lambda(a, b)=\sqrt{\Phi(a) / \Phi(b)}$. Тогда $\Phi_{a, b}\left(z^{(1)}\right) \times$ $\Phi_{a, b}\left(z^{(2)}\right) \equiv(z-a) /(z-b)$ при $z \in D$. Функции вида (71) потребуются нам только в ситуации, когда $a$ фиксировано, а $b \rightarrow a$. Исходя из этого условия выберем в представлении для $\lambda$ ветвь корня так, что $\lambda(a, b)=1+o(1)$ при $b \rightarrow a$; очевидно, что $o(1)$ определяется скоростью стремления $b \rightarrow a: o(1)=O(|a-b|)$.

Положим

$$
\begin{aligned}
\mathcal{F}(\mathbf{z}) & =\Phi(\mathbf{z})^{n} \prod_{j=1}^{m} \Phi_{a_{j}, b_{j}}(\mathbf{z}) \\
& =\Phi(\mathbf{z})^{n} \prod_{j=1}^{m} \lambda\left(a_{j}, b_{j}\right) \frac{\Phi(\mathbf{z})-\Phi\left(b_{j}\right)}{z-b_{j}} \frac{z-a_{j}}{1-\Phi\left(a_{j}\right) \Phi(\mathbf{z})} .
\end{aligned}
$$

Функция $\mathcal{F}$ мероморфна на $\mathfrak{R}$, дивизор $(\mathcal{F})=n \infty^{(2)}-n \infty^{(1)}+$ $\sum_{j=1}^{m} a_{j}^{(1)}-\sum_{j=1}^{m} b_{j}^{(2)}$ и $\mathcal{F}(\mathbf{x}+)=\mathcal{F}(\mathbf{x}-)$ на $Г$. Тем самым, $(\mathcal{F})=$ $(\psi)$ и для функции $H(\mathbf{z})=\psi(\mathbf{z}) / \mathcal{F}(\mathbf{z})$ имеем: $(H)=0$, т.е. $H$ голоморфна и отлична от нуля в $\mathfrak{R} \backslash \Gamma$, а на $Г$ выполняется краевое условие $\rho(x) H(\mathbf{x}+)=H(\mathbf{x}-)$. Отсюда с учетом выбранных нормировок функций $\psi$ и $\mathcal{F}$ :

$$
\mathcal{F}\left(z^{(1)}\right) \mathcal{F}\left(z^{(2)}\right) \equiv \frac{\left(z-a_{1}\right) \cdots\left(z-a_{m}\right)}{\left(z-b_{1}\right) \cdots\left(z-b_{m}\right)}
$$

получаем, что $H\left(z^{(1)}\right) H\left(z^{(2)}\right) \equiv 1$ при $z \in D$. С учетом этого соотношения из краевого условия для функции $H$ на $\Gamma$ вытекает, что $\rho(x) H^{+}(x) H^{-}(x) \equiv 1$ при $x \in \Delta$. 
Таким образом, функция $H(z)=H\left(z^{(1)}\right)$ голоморфна и отлична от нуля в $D$ и удовлетворяет условию: $\rho(x) H^{+}(x) H^{-}(x) \equiv 1$ при $x \in \Delta$. Тогда функция $u(z)=\frac{\log H(z)}{w(z)}$ однозначно определена и голоморфна в $D, u(\infty)=0$ и

$$
u^{+}(x)-u^{-}(x)=-\frac{\log \rho(x)}{w^{+}(x)}=i \frac{\log \rho(x)}{\sqrt{1-x^{2}}}
$$

при $x \in \Delta$. Следовательно, для $u$ справедливо следующее представление интегралом типа Коши:

$$
\begin{aligned}
u(z) & =\frac{1}{2 \pi i} \int_{\Delta} \frac{\log \rho(x)}{x-z} \frac{d x}{w^{+}(x)}=-\frac{1}{2 \pi} \int_{\Delta} \frac{\log \rho(x)}{x-z} \frac{d x}{\sqrt{1-x^{2}}} \\
& =\frac{1}{2 \pi} \int_{\Delta} \frac{\log \rho(x)}{z-x} \frac{d x}{\sqrt{1-x^{2}}} .
\end{aligned}
$$

Тем самым, произведение $w(z) u(z)=S(z)-$ функция Сегё для веса $\rho$. Отсюда вытекает, что

$$
H(z)=e^{w(z) u(z)}=e^{S(z)}=\exp \left\{\frac{\sqrt{z^{2}-1}}{2 \pi} \int_{\Delta} \frac{\log \rho(x)}{z-x} \frac{d x}{\sqrt{1-x^{2}}}\right\} .
$$

В итоге для $\psi$-функции, решающей задачу $\left(\mathcal{R}_{3}\right)$, получаем следующее представление при $\mathbf{z} \in \mathfrak{R} \backslash \Gamma$ :

$$
\psi(\mathbf{z})=\mathcal{F}(\mathbf{z}) H(\mathbf{z}),
$$

где

$$
\begin{aligned}
& H\left(z^{(1)}\right)=\exp \left\{\frac{\sqrt{z^{2}-1}}{2 \pi} \int_{\Delta} \frac{\log \rho(x)}{z-x} \frac{d x}{\sqrt{1-x^{2}}}\right\} \\
& H\left(z^{(2)}\right) \equiv \frac{1}{H\left(z^{(1)}\right)}, \\
& \mathcal{F}\left(z^{(1)}\right)=\Phi(z)^{n} \prod_{j=1}^{m} \lambda\left(a_{j}, b_{j}\right) \frac{\Phi(z)-\Phi\left(b_{j}\right)}{z-b_{j}} \frac{z-a_{j}}{1-\Phi\left(a_{j}\right) \Phi(z)}, \\
& \mathcal{F}\left(z^{(2)}\right) \equiv \frac{\prod_{j=1}^{m}\left(z-a_{j}\right)}{\prod_{j=1}^{m}\left(z-b_{j}\right) \cdot \mathcal{F}\left(z^{(1)}\right)} .
\end{aligned}
$$


Из полученного представления вытекает, что $\psi(z) / z^{n} \rightarrow \varkappa_{n}$ при $z \rightarrow \infty$, где

$$
\begin{aligned}
\varkappa_{n} & =2^{n} e^{S(\infty)} \prod_{j=1}^{m} \frac{\lambda\left(a_{j}, b_{j}\right)}{\left(-\Phi\left(b_{j}\right)\right)} \\
& =2^{n} \exp \left\{\frac{1}{2 \pi} \int_{\Delta} \frac{\log \rho(x) d x}{\sqrt{1-x^{2}}}\right\} \prod_{j=1}^{m} \frac{\lambda\left(a_{j}, b_{j}\right)}{\left(-\Phi\left(b_{j}\right)\right)} \neq 0,
\end{aligned}
$$

т.е. $\psi$ имеет в точке $z=\infty$ полюс $n$-го порядка. Непосредственно проверяется, что полученная функция $\psi$ является решением задачи $\left(\mathcal{R}_{3}\right)$. Отметим также следующее свойство этой функции, которое понадобится нам в дальнейшем:

$$
\rho(x) \psi^{+}(x) \psi^{-}(x) \equiv \prod_{j=1}^{m} \frac{x-a_{j}}{x-b_{j}} \quad \text { при } \quad x \in \Delta .
$$

Наконец, при $b_{j}=a_{j}, j=1, \ldots, m$, полученные результаты прямо приводят к $\Psi$-функции, решающей задачу $\left(\mathcal{R}_{2}\right)$ :

$$
\Psi(\mathbf{z})=\mathcal{F}(\mathbf{z}) H(\mathbf{z}),
$$

где $H(\mathbf{z})$ определяется условиями (73), а

$$
\mathcal{F}\left(z^{(1)}\right)=\Phi(z)^{n} \prod_{j=1}^{m} \frac{\Phi(z)-\Phi\left(a_{j}\right)}{1-\Phi\left(a_{j}\right) \Phi(z)}, \quad \mathcal{F}\left(z^{(2)}\right) \equiv \frac{1}{\mathcal{F}\left(z^{(1)}\right)}
$$

при этом $\rho(x) \Psi^{+}(x) \Psi^{-}(x) \equiv 1$ при $x \in \Delta$ и $\Psi(z) / z^{n} \rightarrow \varkappa_{n}$ при $z \rightarrow \infty$, где

$$
\varkappa_{n}=\frac{2^{n} e^{S(\infty)}}{\prod_{j=1}^{m}\left(-\Phi\left(a_{j}\right)\right)} \neq 0
$$

т.е. $\Psi$ имеет в точке $z=\infty$ полюс $n$-го порядка.

2. Рассуждения этого пункта основаны на свойствах $\psi$-функции. Всюду здесь предполагается, что в условии $2^{\circ}$ задачи $\left(\mathcal{R}_{3}\right)$ точки $a_{j}, j=1, \ldots, m,-$ полюсы $^{10}$ функции $f$ в $D, b_{j}=a_{j, n}-$ полюсы рациональной дроби $f_{n}$, соответствующие полюсам $f$ в $D$

\footnotetext{
${ }^{10}$ Напомним, что в этом параграфе мы придерживаемся следующего соглашения: каждый полюс или нуль функции выписывается столько раз, какова его кратность.
} 
при $n \rightarrow \infty$ и занумерованные так, что $a_{j, n} \rightarrow a_{j}$ при $n \rightarrow \infty$ для $j=1, \ldots, m$. Тем самым дивизор $(\psi)=n \infty^{(2)}+a_{1}^{(1)}+\cdots+a_{m}^{(1)}-$ $a_{1, n}^{(2)}-\cdots-a_{m, n}^{(2)}-n \infty^{(1)}$, а соответствующие $\lambda=\lambda\left(a_{j}, b_{j}\right) \rightarrow 1$ при $n \rightarrow \infty$ с геометрической скоростъю (см. $\S 2$, п. 3). Положим $\omega_{m}(z)=\prod_{j=1}^{m}\left(z-a_{j}\right), \omega_{n, m}(z)=\prod_{j=1}^{m}\left(z-a_{j, n}\right)$.

Определим на $\mathfrak{R} \backslash \Gamma$ функцию $F$ следующим образом:

$$
F(\mathbf{z})=\psi(\mathbf{z}) \cdot \begin{cases}R_{n}(z) w(z), & \mathbf{z}=z^{(1)} \in D^{(1)} \\ -2 Q_{n}(z), & \mathbf{z}=z^{(2)} \in D^{(2)}\end{cases}
$$

Опираясь на свойства функции $\psi$ в $\mathfrak{R} \backslash \Gamma$, нетрудно увидеть, что функция $F$ (кусочно) голоморфна в $\mathfrak{R} \backslash \Gamma$. Как будет показано ниже, все достаточно большие индексы $n$ нормальны для функции $f=\widehat{\rho}+r$ и для таких $n$ дивизор $(F)=0$.

Используя свойства $\psi$-функции на кривой $Г$ и следующее свойство функции остатка $R_{n}$ на границе отрезка $\Delta: R_{n}^{+}(x)-$ $R_{n}^{-}(x)=-2 Q_{n}(x) \rho(x) / w^{+}(x)$, получаем краевое условие для $F$ на $\Gamma$ :

$$
F(\boldsymbol{\zeta}+)-F(\boldsymbol{\zeta}-)=\chi(\boldsymbol{\zeta}-) \frac{1}{\rho(\zeta)}, \quad \boldsymbol{\zeta} \in \Gamma,
$$

где функция $\chi(\mathbf{z})=\psi(\mathbf{z}) R_{n}(z) w(\mathbf{z}), \mathbf{z} \in D^{(2)}$, мероморфна на втором листе. В таком случае $F$ стандартным образом (см. [19], [16]) восстанавливается в $\mathfrak{R} \backslash \Gamma$ по граничным условиям (78) интегралом типа Коши:

$$
F(\mathbf{z})=-\frac{1}{2 \pi i} \int_{\Gamma^{+}} \chi(\boldsymbol{\zeta}-) \frac{1}{\rho(\zeta)} d \Omega(\boldsymbol{\zeta} ; \mathbf{z})+C_{n}, \quad \mathbf{z} \notin \Gamma,
$$

где $C_{n}-$ некоторая постоянная,

$$
d \Omega(\boldsymbol{\zeta} ; \mathbf{z})=\frac{1}{2} \frac{w(\boldsymbol{\zeta})+w(\mathbf{z})}{\zeta-z} \frac{d \zeta}{w(\boldsymbol{\zeta})}
$$

- мероморфный на $\mathfrak{R}$ абелев дифференциал, а контур $\Gamma^{+}$ориентирован положительно относительно области $D^{(1)}$ : область остается слева при обходе по контуру. Как обычно, дальнейшие рассуждения основаны на интегральном представлении (79).

Пусть сначала $\mathbf{z}=z^{(1)} \in D^{(1)}$. Выберем числа $R>1$ и $\varepsilon>0$ так, чтобы функции $\chi$ и $1 / \rho$ были бы голоморфны в области 
$\left\{z^{(2)}: 1<|\Phi(z)|<R+\varepsilon\right\}$. Тогда в интегральном представлении (79) контур $\Gamma$ можно заменить на контур $\Gamma_{R}^{(2)} \subset D^{(2)}$. Воспользовавшись тем, что

$$
\begin{aligned}
\chi\left(z^{(2)}\right) & =\psi\left(z^{(2)}\right) R_{n}(z) w\left(z^{(2)}\right)=-F\left(z^{(1)}\right) \frac{\psi\left(z^{(2)}\right)}{\psi\left(z^{(1)}\right)} \\
& =-F\left(z^{(1)}\right) \frac{\omega_{m}(z) / \omega_{m, n}(z)}{\psi\left(z^{(1)}\right)^{2}}
\end{aligned}
$$

и $d \Omega\left(\zeta^{(2)} ; z^{(1)}\right)=d \Omega\left(\zeta^{(1)} ; z^{(2)}\right)$, получаем из $(79)$ следующее представление для функции $F$ :

$$
\begin{aligned}
F\left(z^{(1)}\right) & =-\frac{1}{2 \pi i} \int_{\Gamma_{R}^{(2)}} \chi\left(\zeta^{(2)}\right) \frac{1}{\rho(\zeta)} d \Omega\left(\zeta^{(2)} ; z^{(1)}\right)+C_{n} \\
& =-\frac{1}{2 \pi i} \int_{\Gamma_{R}^{(1)}} F\left(\zeta^{(1)}\right) \frac{\omega_{m}(z) / \omega_{m, n}(z)}{\psi\left(\zeta^{(1)}\right)^{2}} \frac{1}{\rho(\zeta)} d \Omega\left(\zeta^{(1)} ; z^{(2)}\right)+C_{n},
\end{aligned}
$$

где $\Gamma_{R}^{(1)}=\left\{z^{(1)}:|\Phi(z)|=R\right\}$. Так как дифференциал $d \Omega\left(\zeta^{(1)} ; \mathbf{z}\right)$ - голоморфный по $\mathbf{z}$ на компакте $D^{(2)} \sqcup \Gamma$ при $\zeta^{(1)} \in \Gamma_{R}^{(1)}$, то последнее интегральное представление ${ }^{11}$ справедливо равномерно на компакте $D^{(1)} \sqcup \Gamma$. Из (81) получаем, что равномерно по $\mathbf{z} \in D^{(1)} \sqcup \Gamma$

$$
\left|F(\mathbf{z})-C_{n}\right|=M_{n} \max _{\Gamma_{R}} \frac{1}{\left|\psi\left(\zeta^{(1)}\right)\right|^{2}} O(1) \quad \text { при } n \rightarrow \infty,
$$

где $M_{n}=\max _{\Gamma_{R}^{(1)}}\left|F\left(\zeta^{(1)}\right)\right|$. Используя свойства $\psi$-функции, получаем отсюда, что равномерно по $\mathbf{z} \in D^{(1)} \sqcup \Gamma$

$$
\left|F(\mathbf{z})-C_{n}\right|=M_{n} \cdot O\left(\delta^{n}\right) \quad \text { при } n \rightarrow \infty,
$$

где $\delta=1 / R^{2}<1$. Так как $F\left(z^{(1)}\right) \not \equiv 0$, то $M_{n} \neq 0$, поэтому из $(82)$ последовательно получаем: $M_{n}=\left|C_{n}\right|\left(1+O\left(\delta^{n}\right)\right)$ и

$$
F\left(z^{(1)}\right)=C_{n}\left(1+O\left(\delta^{n}\right)\right) \quad \text { при } n \rightarrow \infty
$$

равномерно по $\mathbf{z} \in D^{(1)} \sqcup \Gamma$.

\footnotetext{
${ }^{11}$ Как и ранее, интегральная формула (81) задает аналитическое продолжение функции $F(\mathbf{z}), \mathbf{z} \in D^{(1)}$, через контур $Г$ на второй лист римановой поверхности $\Re$ вплоть до контура $\Gamma_{R}^{(2)}$, причем для $\mathbf{z} \in D^{(2)}$ это продолжение, вообще говоря, не совпадает с функцией $F(\mathbf{z}), \mathbf{z} \in D^{(2)}$, определенной (77).
} 
Пусть теперь $\mathbf{z}=z^{(2)} \in D^{(2)}$. Тогда из (79) вытекает, что при $z^{(2)} \in D_{R}^{(2)}=\left\{z^{(2)}:|\Phi(z)|>R\right\}$

$$
\begin{aligned}
F\left(z^{(2)}\right) & =-\frac{1}{2 \pi i} \int_{\Gamma_{R}^{(2)}} \chi\left(\zeta^{(2)}\right) \frac{1}{\rho(\zeta)} d \Omega\left(\zeta^{(2)} ; z^{(2)}\right)+C_{n} \\
& =-\frac{1}{2 \pi i} \int_{\Gamma_{R}^{(1)}} F\left(\zeta^{(1)}\right) \frac{\omega_{m}(z) / \omega_{m, n}(z)}{\psi\left(\zeta^{(1)}\right)^{2}} \frac{1}{\rho(\zeta)} d \Omega\left(\zeta^{(1)} ; z^{(1)}\right)+C_{n} .
\end{aligned}
$$

Дифференциал $d \Omega\left(\zeta^{(1)} ; z^{(1)}\right)$ - голоморфный по $z^{(1)} \in D_{R}^{(1)}$ при $\zeta^{(1)} \in \Gamma_{R}^{(1)}$. Используя свойства $\psi$-функции и полученное выше асимптотическое представление (83) для функции $F(\mathbf{z})$, получаем из (84) аналог соотношения (83) для $F\left(z^{(2)}\right)$ при $n \rightarrow \infty$ :

$$
F\left(z^{(2)}\right)=C_{n}\left(1+O\left(\delta^{n}\right)\right)
$$

равномерно внутри $D_{R}^{(2)}, \delta<1$. Непосредственно из определения (77) функции $F$ вытекает, что равномерно внутри $D_{R}, R>1$, справедливо представление

$$
2 Q_{n}(z)=-C_{n} \frac{1}{\psi\left(z^{(2)}\right)}(1+o(1)), \quad \text { где } o(1)=O\left(\delta^{n}\right), \quad \delta<1 .
$$

Так как в силу (83) постоянная $C_{n} \neq 0$ при достаточно больших $n$, то $\operatorname{deg} Q_{n}=n$, т.е. все такие индексы нормальны. Выбор постоянной $C_{n} \neq 0$ связан только с нормировкой $Q_{n}$, поэтому в дальнейшем положим $C_{n}=-2$. В таком случае асимптотическая формула для полиномов $Q_{n}$ вне отрезка $\Delta$ примет вид:

$Q_{n}(z)=\frac{1}{\psi\left(z^{(2)}\right)}(1+o(1))=\psi(z) \prod_{j=1}^{m} \frac{\omega_{m, n}(z)}{\omega_{m}(z)}(1+o(1)), \quad n \rightarrow \infty$ или, окончательно,

$$
Q_{n}(z) \frac{\omega_{m}(z)}{\omega_{m, n}(z)}=\psi(z)(1+o(1))
$$

равномерно внутри $D$ при $n \rightarrow \infty$.

Вернемся теперь к соотношению

$$
F(\mathbf{z})=C_{n}(1+o(1))=-2+o(1)
$$


которое справедливо равномерно на компакте $D^{(1)} \sqcup \Gamma$. Из этого соотношения в силу определения функции $F$ вытекает, что равномерно по $x \in \Delta$

$$
\begin{aligned}
& \psi^{+}(x) R^{+}(x) w^{+}(x)=-2+o(1), \quad n \rightarrow \infty . \\
& \psi^{-}(x) R^{-}(x) w^{-}(x)=-2+o(1),
\end{aligned}
$$

Так как $\psi^{+}(x) \neq 0$ и $\psi^{-}(x) \neq 0$ на $\Delta$, то

$$
\begin{aligned}
& R^{+}(x) w^{+}(x)=-\frac{2}{\psi^{+}(x)}(1+o(1)), \quad n \rightarrow \infty . \\
& R^{-}(x) w^{-}(x)=-\frac{2}{\psi^{-}(x)}(1+o(1)),
\end{aligned}
$$

Суммируя последние соотношения, получаем

$$
R_{n}^{+}(x) w^{+}(x)+R_{n}^{-}(x) w^{-}(x)=-\frac{2}{\psi^{+}(x)}-\frac{2}{\psi^{-}(x)}+o(1) .
$$

Поскольку $R_{n}^{+}(x)-R_{n}^{-}(x)=-2 Q_{n}(x) \rho(x) / w^{+}(x)$ при $x \in \Delta$, то $R_{n}^{+}(x) w^{+}(x)+R_{n}^{-}(x) w^{-}(x)=-2 Q_{n}(x) \rho(x)$. Объединяя последние соотношения для функции остатка, получаем, что

$$
Q_{n}(x) \rho(x)=\frac{1}{\psi^{+}(x)}+\frac{1}{\psi^{-}(x)}+o(1) .
$$

Но $\rho(x) \psi^{+}(x) \psi^{-}(x) \equiv \omega_{m}(x) / \omega_{m, n}(x)=1+o(1)$ на $\Delta$, тем самым

$$
Q_{n}(x) \frac{\omega_{m}(x)}{\omega_{m, n}(x)}=\psi^{+}(x)+\psi^{-}(x)+o(1)
$$

равномерно по $x \in \Delta$, где $o(1)=o\left(\delta^{n}\right), \delta<1$.

Итак, получены (см. выше (86) и (87)) следующие асимптотические представления для полиномов $Q_{n}(z)=Q_{n}(z ; f)$ :

$$
\begin{gathered}
Q_{n}(z ; f) \frac{\omega_{m}(z)}{\omega_{m, n}(z)}=\psi(z)\left(1+o\left(\delta^{n}\right)\right) \\
\text { равномерно внутри } D^{\prime}, \\
Q_{n}(x ; f) \frac{\omega_{m}(x)}{\omega_{m, n}(x)}=\psi^{-}(x)+\psi^{+}(x)+o\left(\delta^{n}\right) \\
\text { равномерно на } \Delta,
\end{gathered}
$$


где $\omega_{m}(z)=\prod_{j=1}^{m}\left(z-a_{j}\right), \omega_{m, n}(z)=\prod_{j=1}^{m}\left(z-a_{j, n}\right)$, а $\delta<1$. Так как равномерно внутри $D^{\prime}$ и равномерно на $\Delta$ при $n \rightarrow \infty$ имеем: $\omega_{m, n}(z)=\omega_{m}(z)\left(1+o\left(\delta^{n}\right)\right)$ и $\psi(z)=\Psi(z)\left(1+o\left(\delta^{n}\right)\right)$, где $\Psi-$ решение задачи $\left(\mathcal{R}_{2}\right)$, то из $(88)$ немедленно следуют утверждения предложения 2 .

Предложение 2 доказано.

В дальнейшем нам понадобится также следующее соотношение, которое вытекает непосредственно из (88) и представления (75), (76):

$$
\frac{Q_{n}(z ; f)}{\omega_{m, n}(z)}=\frac{\Psi(z)}{\omega_{m}(z)}\left(1+o\left(\delta^{n}\right)\right) \quad \text { равномерно внутри } D,
$$

или, если ввести обозначения $\widetilde{Q}_{n}(z ; f)=Q_{n}(z ; f) / \omega_{m, n}(z), \widetilde{\Psi}(z)=$ $\Psi(z) / \omega_{m}(z)$, то

$$
\widetilde{Q}_{n}(z ; f)=\widetilde{\Psi}(z)\left(1+o\left(\delta^{n}\right)\right) \quad \text { равномерно внутри } D \text {. }
$$

\section{§6. Доказательство теоремы 3}

1. Доказательство теоремы 3 основано, главным образом, на формуле сильной асимптотики $\left(22_{2}\right)$ полиномов $Q_{n}(z)=Q_{n}(z ; f)$ на отрезке $\Delta$. Наша ближайшая цель - придать этой формуле вид, пригодный для дальнейших рассуждений. Для отображающей функции $\Phi(z)=z+\sqrt{z^{2}-1}$ имеем:

$$
\Phi^{+}(x)=e^{i g^{*+}(x)}=e^{i \arccos x}, \quad \Phi^{-}(x)=e^{-i \arccos x} \quad \text { при } x \in \Delta .
$$

Следовательно, $\left|\Phi^{+}(x)\right| \equiv\left|\Phi^{-}(x)\right| \equiv 1$ на $\Delta$. Учитывая теперь представление (75), (76) для $\Psi$-функции, получаем: $\Psi^{+}(x)=O(1)$ и $\Psi^{-}(x)=O(1)$ при $n \rightarrow \infty$ равномерно на $\Delta$. Так как $Q_{n}(x)=$ $\Psi^{+}(x)+\Psi^{-}(x)+o(1)$ равномерно на отрезке $\Delta$, то, во-первых, $Q_{n}(x)=O(1)$ при $n \rightarrow \infty$ равномерно по $x \in \Delta$, а во-вторых, $Q_{n}^{2}(x)=\Psi^{+}(x)^{2}+\Psi^{-}(x)^{2}+\frac{2}{\rho(x)}+o(1), \quad$ где $o(1)=o\left(\delta^{n}\right), \quad \delta<1 ;$

здесь мы воспользовались полученным ранее соотношением

$$
\rho(x) \Psi^{+}(x) \Psi^{-}(x) \equiv 1
$$

при $x \in \Delta$. 
Пусть $a_{1}, \ldots, a_{\ell}-$ (различные) полюсы функции $f$ в области $D, m_{j}, j=1, \ldots, \ell,-$ их кратности, $m=\sum_{j=1}^{\ell} m_{j}-$ полное число полюсов $f$ в $D$. Положим $\omega_{m}(z)=\prod_{j=1}^{\ell}\left(z-a_{j}\right)^{m_{j}}$, $\omega_{n, m}(z)=\prod_{j=1}^{m}\left(z-a_{j, n}\right)$, где $a_{j, n}, j=1, \ldots, m,-$ соответствующие полюсам $f$ при $n \rightarrow \infty$ полюсы рациональной дроби $f_{n}$; пусть $\widetilde{Q}_{n}(z)=Q_{n}(z) / \omega_{n, m}(z)$, тогда $\operatorname{deg} \widetilde{Q}_{n}=n-m$ при достаточно больших $n$. Умножив соотношение (4) на полином $\widetilde{Q}_{n} \omega_{m}$ степени $n$, получим

$$
\widetilde{R}_{n}(z)=\left(Q_{n} \widetilde{Q}_{n} \omega_{m} f-\widetilde{P}_{n}\right)(z)=O\left(\frac{1}{z}\right) \quad \text { при } n \rightarrow \infty
$$

где функция $\widetilde{R}_{n}=R_{n} \widetilde{Q}_{n} \omega_{m}$ голоморфна в $D, \widetilde{P}_{n}=P_{n} \widetilde{Q}_{n} \omega_{m}$. Для голоморфной в $D$ функции $\widetilde{R}_{n}$ справедливо представление

$$
\begin{aligned}
\widetilde{R}_{n}(z) & =\frac{1}{\pi} \int_{\Delta} \frac{Q_{n}(x) \widetilde{Q}_{n}(x) \omega_{m}(x) \rho(x) d x}{(z-x) \sqrt{1-x^{2}}} \\
& =\frac{1}{\pi} \int_{\Delta} \frac{Q_{n}^{2}(x)\left(1+\delta_{n}(x)\right) \rho(x) d x}{(z-x) \sqrt{1-x^{2}}} \\
& =\frac{1}{\pi} \int_{\Delta} \frac{Q_{n}^{2}(x) \rho(x) d x}{(z-x) \sqrt{1-x^{2}}}+\frac{1}{\pi} \int_{\Delta} \frac{Q_{n}^{2}(x) \delta_{n}(x) \rho(x) d x}{(z-x) \sqrt{1-x^{2}}} \\
& =I_{1}(z)+I_{2}(z), \quad z \in D,
\end{aligned}
$$

где $\varlimsup_{n \rightarrow \infty}\left\|\delta_{n}\right\|_{\Delta}^{1 / n}<1$. Так как $Q_{n}^{2}(x)=O(1)$ при $n \rightarrow \infty$ равномерно по $x \in \Delta$, то для

$$
I_{2}(z)=\frac{1}{\pi} \int_{\Delta} \frac{Q_{n}^{2}(x) \delta_{n}(x) \rho(x) d x}{(z-x) \sqrt{1-x^{2}}}
$$

имеем

$$
I_{2}(z)=\frac{o(1)}{\sqrt{z^{2}-1}}=G^{\prime}(z) o(1)
$$

при $n \rightarrow \infty$ равномерно внутри $D$,

где $o(1)=o\left(\delta^{n}\right), \delta<1$. Займемся теперь асимптотикой величины

$$
I_{1}(z)=\frac{1}{\pi} \int_{\Delta} \frac{Q_{n}^{2}(x)}{(z-x)} \frac{\rho(x) d x}{\sqrt{1-x^{2}}} .
$$


Дальнейшие рассуждения основаны на асимптотической формуле (90) для $Q_{n}^{2}$ на отрезке $\Delta$.

Последовательно используя (90) и свойства $\Psi$-функции, преобразуем представление для $I_{1}(z)$ следующим образом:

$$
\begin{aligned}
& I_{1}(z)= \frac{1}{\pi} \int_{\Delta} \frac{2}{z-x} \frac{d x}{\sqrt{1-x^{2}}} \\
&+\frac{1}{\pi} \int_{\Delta} \frac{\Psi^{+}(x)^{2}+\Psi^{-}(x)^{2}}{z-x} \frac{\rho(x) d x}{\sqrt{1-x^{2}}}+\frac{o(1)}{\sqrt{z^{2}-1}} \\
&= \frac{2}{\sqrt{z^{2}-1}}+\frac{1}{\pi} \int_{\Delta}\left(\frac{1}{\Psi^{-}(x)^{2}}+\frac{1}{\Psi^{+}(x)^{2}}\right) \frac{d x}{(z-x) \rho(x) \sqrt{1-x^{2}}} \\
&+\frac{o(1)}{\sqrt{z^{2}-1}} \\
&= \frac{2+o(1)}{\sqrt{z^{2}-1}}+\frac{1}{2 \pi i} \int_{\Gamma_{R}} \frac{1}{\zeta-z} \frac{d \zeta}{\Psi(\zeta)^{2} \rho(\zeta) \sqrt{\zeta^{2}-1}} \\
&= \frac{2}{\sqrt{z^{2}-1}}+\frac{o(1)}{\sqrt{z^{2}-1}}=\frac{2}{\sqrt{z^{2}-1}}(1+o(1)) \\
&= 2 G^{\prime}(z)(1+o(1)) \\
& \text { при } \quad n \rightarrow \infty \text { равномерно внутри } D_{R}, \quad R>1,
\end{aligned}
$$

где $D_{R}=\{\zeta:|\Phi(\zeta)|>R\}, \quad \Gamma_{R}=\partial D_{R}, \quad R>1, o(1)=o\left(\delta^{n}\right)$, $\delta<1$. Таким образом,

$$
\widetilde{R}_{n}(z)=2 G^{\prime}(z)(1+o(1)) \quad \text { при } n \rightarrow \infty \text { равномерно внутри } D \text {. }
$$

Непосредственно из хода доказательства этого соотношения видно, что оно "выдерживает" дифференцирование любое число раз: для любого целого $k \geqslant 1$

$$
\begin{gathered}
\widetilde{R}_{n}^{(k)}(z)=2 G^{(k+1)}(z)(1+o(1)) \\
\text { при } n \rightarrow \infty \text { равномерно внутри } D ;
\end{gathered}
$$

последнее соотношение важно для нас лишь постольку, поскольку его правая часть равномерно ограничена внутри $\mathbb{C} \backslash \Delta$, а следовательно, - и в окрестности полюсов функции $f$ в $D$. 
2. Напомним, что $\widetilde{R}_{n}(z)=\left(Q_{n} \widetilde{Q}_{n} \omega_{m} f-\widetilde{P}_{n}\right)(z)=\left(Q_{n} \widetilde{Q}_{n} F-\right.$ $\left.\widetilde{P}_{n}\right)(z)$, где функция $F=\omega_{m} f$ голоморфна в $D$, а полином $\widetilde{P}_{n}=$ $P_{n} \widetilde{Q}_{n} \omega_{m}$. Величина $A_{j}=F\left(a_{j}\right) \neq 0, j=1, \ldots, \ell$. Зафиксируем теперь произвольное $j \in\{1, \ldots, \ell\}$ и, тем самым, соответствующие ему $a_{j}$ и $m_{j}$. В силу соотношений $(92),(93)$ величина $\widetilde{R}_{n}^{(k)}\left(a_{j}\right)=$ $2 G^{(k+1)}\left(a_{j}\right)(1+o(1))$ для $k=0, \ldots, m_{j}-1$ при $n \rightarrow \infty$. С другой стороны, для таких $k$ величина $\widetilde{P}_{n}^{(k)}\left(a_{j}\right)=\left(P_{n} \widetilde{Q}_{n} \omega_{m}\right)\left(a_{j}\right)^{(k)}=0$, поэтому в силу формулы Лейбница

$$
\begin{aligned}
\widetilde{R}_{n}^{(k)}\left(a_{j}\right) & =\left(Q_{n} \widetilde{Q}_{n} F\right)^{(k)}\left(a_{j}\right)=\left(\omega_{m, n} \widetilde{Q}_{n}^{2} F\right)^{(k)}\left(a_{j}\right) \\
& =A_{j} \widetilde{Q}_{n}^{2}\left(a_{j}\right) \omega_{m, n}^{(k)}\left(a_{j}\right)+\sum_{s=1}^{k}\left(\begin{array}{c}
k \\
s
\end{array}\right)\left(\widetilde{Q}_{n}^{2} F\right)^{(s)} \omega_{m, n}^{(k-s)}\left(a_{j}\right) .
\end{aligned}
$$

Подставляя это соотношение при $k=0$ в (92), получаем $A_{j} \widetilde{Q}_{n}^{2}\left(a_{j}\right) \omega_{m, n}\left(a_{j}\right)=2 G^{\prime}\left(a_{j}\right)(1+o(1)), \quad n \rightarrow \infty, \quad$ где $G^{\prime}\left(a_{j}\right) \neq 0$,

аналогично из $(93)$ для $k=1, \ldots, m_{j}-1$ имеем:

$$
\begin{aligned}
& A_{j} \widetilde{Q}_{n}^{2}\left(a_{j}\right) \omega_{m, n}^{(k)}\left(a_{j}\right)+\sum_{s=1}^{k}\left(\begin{array}{l}
k \\
s
\end{array}\right)\left(\widetilde{Q}_{n}^{2} F\right)^{(s)} \omega_{m, n}^{(k-s)}\left(a_{j}\right) \\
& =2 G^{(k+1)}\left(a_{j}\right)(1+o(1)) ;
\end{aligned}
$$

в частности,

$$
A_{j} \widetilde{Q}_{n}^{2}\left(a_{j}\right) \omega_{m, n}^{\prime}\left(a_{j}\right)+\left(\widetilde{Q}_{n}^{2} F\right)^{\prime} \omega_{m, n}\left(a_{j}\right)=2 G^{\prime \prime}\left(a_{j}\right)(1+o(1)) .
$$

Пусть $a_{j, \nu}(n), \nu=1, \ldots, m_{j},-$ полюсы рациональной дроби $f_{n}$, соответствующие при $n \rightarrow \infty$ точке $a_{j}$. Положим $q_{n}(z)=$ $\prod_{\nu=1}^{m_{j}}\left(z-a_{j, \nu}(n)\right), \quad \widetilde{\omega}_{n}(z)=\omega_{m, n}(z) / q_{n}(z)$. Тогда $\widetilde{\omega}_{n}\left(a_{j}\right)=\alpha_{j}(1+$ $o(1))$ при $n \rightarrow \infty$, где $\alpha_{j}=\omega_{m}^{\left(m_{j}\right)}\left(a_{j}\right) / m_{j} ! \neq 0$. Тем самым, величина $A_{j} \alpha_{j} \widetilde{\Psi}\left(a_{j}\right)^{2} / 2 G^{\prime}\left(a_{j}\right) \neq 0, \infty$; здесь $\widetilde{\Psi}=\Psi / \omega_{m}, G^{\prime}\left(a_{j}\right) \neq 0$. Пусть

$$
\lambda_{j}=\lambda_{j}(n)=-\left(-\frac{A_{j} \alpha_{j} \widetilde{\Psi}\left(a_{j}\right)^{2}}{2 G^{\prime}\left(a_{j}\right)}\right)^{2 / m_{j}} .
$$

Тогда

$$
\begin{aligned}
\lambda_{j}=- & \left|\Phi\left(a_{j}\right)\right|^{2 n / m_{j}} \exp \left\{\frac{i 2 n g^{*}\left(a_{j}\right)}{m_{j}}\right\} \exp \left\{\frac{2 S\left(a_{j}\right)}{m_{j}}\right\} \\
& \times\left(-\frac{A_{j} \alpha_{j}}{2 G^{\prime}\left(a_{j}\right)} \frac{\Pi_{m}}{\omega_{m}}\left(a_{j}\right)\right)^{2 / m_{j}}
\end{aligned}
$$


и из $(94)$ вытекает, что $q_{n}\left(a_{j}\right) \lambda_{j}^{m_{j}}=1+o(1)$ при $n \rightarrow \infty$, где $o(1)=o\left(\delta^{n}\right)$. Так как для достаточно больших $n$ все нули полиномов $\widetilde{Q}_{n}$ и их производных лежат в произвольной наперед заданной окрестности $U$ отрезка $\Delta$, множество $\left\{a_{1}, \ldots, a_{\ell}\right\} \subset \mathbb{C} \backslash \bar{U}$, TO

$$
\left|\frac{\left(F \widetilde{Q}_{n}^{2}\right)^{\prime}(z)}{\widetilde{Q}_{n}^{2}(z)}\right|=O(n) \quad \text { при } n \rightarrow \infty \text { равномерно внутри } \mathbb{C} \backslash \bar{U}
$$

Рассуждая по индукции, получаем отсюда, что и для любого $k=$ $2, \ldots, m_{j}$

$$
\left|\frac{\left(F \widetilde{Q}_{n}^{2}\right)^{(k)}(z)}{\widetilde{Q}_{n}^{2}(z)}\right|=O\left(n^{k}\right) \quad \text { при } n \rightarrow \infty \text { равномерно внутри } \mathbb{C} \backslash \bar{U}
$$

Модуль величины $\lambda_{j}(n)$ растет со скоростью геометрической прогрессии при $n \rightarrow \infty$ :

$$
\lim _{n \rightarrow \infty}\left|\lambda_{j}(n)\right|^{1 / n}=\left|\Phi\left(a_{j}\right)\right|^{2 / m_{j}}>1
$$

Поэтому из (96) вытекает, что $q_{n}^{\prime}\left(a_{j}\right) \lambda_{j}^{m_{j}-1}=\left(q_{n}^{\prime}\left(a_{j}\right) \lambda_{j}^{m_{j}}\right) / \lambda_{j}=o(1)$ при $n \rightarrow \infty$, где, как обычно, $o(1)=o\left(\delta^{n}\right), \delta<1$. Вновь рассуждая по индукции, получаем из $(94)$ и $(95)$, что $q_{n}^{(k)}\left(a_{j}\right) \lambda_{j}^{m_{j}-k}=o(1)$ при $n \rightarrow \infty$ для $k=2, \ldots, m_{j}-1$.

Пусть теперь $p_{n}(z)=\prod_{\nu=1}^{m_{j}}\left(z-z_{\nu, n}\right)$, где $z_{\nu, n}=\lambda_{j}(n)\left(a_{j}-\right.$ $\left.a_{j, \nu}(n)\right), \nu=1, \ldots, m_{j}$. Тогда из установленных выше асимптотических соотношений вытекает, что $p_{n}(z)=z^{m_{j}}-1+o(1)$. Следовательно, при надлежащей перенумерации точек $z_{\nu, n}-$ нулей $p_{n}-$ имеем: $z_{\nu, n}=\varepsilon_{j}^{\nu}+o(1)$ при $n \rightarrow \infty$, где $\varepsilon_{j}=e^{2 \pi i / m_{j}}, o(1)=o\left(\delta^{n}\right)$. Отсюда уже получаем, что

$$
a_{j, \nu}(n)=a_{j}+\frac{C_{j} \varepsilon_{j}^{\nu}}{B_{j}^{2 n}}(1+o(1))
$$

при $n \rightarrow \infty, \quad \nu=1, \ldots, m_{j}, \quad j=1, \ldots, \ell$. 
Здесь

$$
\begin{aligned}
\varepsilon_{j}= & e^{2 \pi i / m_{j}}, \\
B_{j}= & \Phi\left(a_{j}\right)^{1 / m_{j}}=\left|\Phi\left(a_{j}\right)\right|^{1 / m_{j}} e^{i g^{*}\left(a_{j}\right) / m_{j}} \\
\left|B_{j}\right|= & \left|\Phi\left(a_{j}\right)\right|^{1 / m_{j}}>1 \\
C_{j}= & e^{-2 S\left(a_{j}\right) / m_{j}}\left(-\frac{2 G^{\prime}\left(a_{j}\right)}{A_{j} \alpha_{j}}\right)^{1 / m_{j}} \\
& \quad \times \frac{(-2)^{m / m_{j}} \Phi\left(a_{j}\right)^{m / m_{j}} \prod_{s=1}^{\ell} \Phi\left(a_{s}\right)^{m_{s} / m_{j}}}{\prod_{s=1}^{\ell}\left(1-\Phi\left(a_{s}\right) \Phi\left(a_{j}\right)\right)^{2 m_{s} / m_{j}}}
\end{aligned}
$$

а величины $A_{j}$ и $\alpha_{j}, j=1, \ldots, \ell$, конечны, отличны от нуля и зависят только от $f$ :

$$
A_{j}=\left(f \omega_{m}\right)\left(a_{j}\right) \neq 0, \quad \alpha_{j}=\frac{\omega_{m}^{\left(m_{j}\right)}\left(a_{j}\right)}{m_{j} !} \neq 0, \quad j=1, \ldots, \ell .
$$

Постоянная $C_{j}$ связана с геометрическим расположением $j$-го полюса функции $f$ относительно отрезка $\Delta$ и других полюсов $f$; величина $1 / B_{j}$ характеризует (геометрическую) скорость сходимости полюсов $f_{n}$ к точке $a_{j}$ при $n \rightarrow \infty$ и не зависит от других полюсов $f$; наличие $\varepsilon_{j}, \varepsilon_{j}^{m_{j}}=1$, обеспечивает то асимптотически правильное расположение полюсов $f_{n}$ по отношению к притягивающей точке $a_{j}$, о котором говорилось во введении.

Теорема 3 доказана.

Формулы (97), (98) полностью описывают асимптотическое поведение полюсов рациональных дробей $f_{n}$, находящихся вблизи полюсов $f$ в $D$.

3. Перейдем к доказательству следствий.

Справедливость следствия 1 вытекает непосредственно из хода доказательства теоремы 3 (см. формулы (91)-(93)).

Утверждение следствия 2 вытекает из того, что в его условиях при всех достаточно больших $n$ на отрезке $\Delta$ расположены $n-$ $m$ простых нулей полинома $Q_{n}(x ; f)$. Поскольку в силу теоремы 3 для оставшихся $m$ нулей справедливы асимптотические формулы вида (97), (98), то при $j=1, \ldots, \ell$ и $\nu, \mu=0, \ldots, m_{j}-1, \quad \nu \neq \mu$, имеем

$$
\left|a_{j, \nu}(n)-a_{j, \mu}(n)\right|=\frac{\left|C_{j}\right| \cdot\left|\varepsilon_{j}^{\nu-\mu}-1\right|}{\left|B_{j}^{n}\right|}(1+o(1)) \quad \text { при } n \rightarrow \infty .
$$


Так как $\varepsilon_{j}^{\nu-\mu} \neq 1$ при $\nu \neq \mu$, то из последней асимптотической формулы вытекает, что при достаточно больших $n$ и оставшиеся $m$ нулей знаменателя $f_{n}$ простые.

Докажем следствие 3. Из теоремы Сохоцкого получаем, что для $x \in \Delta$

$$
\widehat{\rho}^{+}(x)=I(x)+i \frac{\rho(x)}{\sqrt{1-x^{2}}}, \quad \widehat{\rho}^{-}(x)=I(x)-i \frac{\rho(x)}{\sqrt{1-x^{2}}},
$$

где

$$
I(x)=\frac{1}{\pi} \int_{\Delta} \frac{\rho(t)-\rho(x)}{x-t} \frac{d t}{\sqrt{1-t^{2}}} .
$$

Аналогично, для функции

$$
u(z)=\frac{1}{\pi} \int_{\Delta} \frac{\log \rho(t)}{z-t} \frac{d t}{\sqrt{1-t^{2}}}
$$

имеем

$$
\begin{aligned}
& u^{+}(x)=J(x)-\frac{\log \rho(x)}{w^{+}(x)}=J(x)+\frac{i \log \rho(x)}{\sqrt{1-x^{2}}}, \\
& u^{-}(x)=J(x)-\frac{i \log \rho(x)}{\sqrt{1-x^{2}}},
\end{aligned}
$$

где

$$
J(x)=\frac{1}{\pi} \int_{\Delta} \frac{\log \rho(t)-\log \rho(x)}{x-t} \frac{d t}{\sqrt{1-t^{2}}} .
$$

Положим $\theta(x)=\sqrt{1-x^{2}} J(x) / 2$. Тогда в условиях следствия 3 величина $\theta(x)$ вещественна при всех $x \in \Delta$, а для функции Сегё $S(z)=w(z) u(z) / 2$ и функции $H(z)=\exp \{S(z)\}$ получаем

$$
\begin{array}{ll}
S^{+}(x)=i \theta(x)-\frac{1}{2} \log \rho(x), & S^{-}(x)=-i \theta(x)-\frac{1}{2} \log \rho(x), \\
H^{+}(x)=\frac{1}{\sqrt{\rho(x)}} \exp \{i \theta(x)\}, & H^{-}(x)=\frac{1}{\sqrt{\rho(x)}} \exp \{-i \theta(x)\} .
\end{array}
$$

Для функции $\mathcal{F}$ (см. (76)) при $x \in \Delta$ имеем с учетом принятого здесь соглашения относительно полюсов функции $f$ :

$$
\begin{aligned}
& \mathcal{F}^{+}(x)=e^{i n \varphi} \prod_{j=1}^{\ell}\left(\frac{e^{i \varphi}-\Phi\left(a_{j}\right)}{1-\Phi\left(a_{j}\right) e^{i \varphi}}\right)^{m_{j}}, \\
& \mathcal{F}^{-}(x)=e^{-i n \varphi} \prod_{j=1}^{\ell}\left(\frac{e^{-i \varphi}-\Phi\left(a_{j}\right)}{1-\Phi\left(a_{j}\right) e^{-i \varphi}}\right)^{m_{j}},
\end{aligned}
$$


где $\varphi=\varphi(x)=\arccos x$. Нетрудно видеть, что так как рациональная функция $r$ вещественна, то при $x \in \Delta$

$$
\mathcal{F}^{+}(x)=e^{n i \varphi(x)+i \alpha(x)}, \quad \mathcal{F}^{-}(x)=e^{-n i \varphi(x)-i \alpha(x)},
$$

где $\alpha(x) \in \mathbb{R}$ при $x \in \Delta$,

$$
\begin{aligned}
\cos \alpha(x)=\frac{1}{2} & \left\{\prod_{j=1}^{\ell}\left(\frac{e^{i \arccos x}-\Phi\left(a_{j}\right)}{1-\Phi\left(a_{j}\right) e^{i \arccos x}}\right)^{m_{j}}\right. \\
& \left.+\prod_{j=1}^{\ell}\left(\frac{e^{-i \arccos x}-\Phi\left(a_{j}\right)}{1-\Phi\left(a_{j}\right) e^{-i \arccos x}}\right)^{m_{j}}\right\} .
\end{aligned}
$$

Перепишем асимптотическую формулу для полиномов $Q_{n}$ на отрезке $\Delta$ следующим образом:

$$
\begin{aligned}
Q_{n}(x ; f) & =\Psi^{+}(x)+\Psi^{-}(x)+o(1) \\
& =\mathcal{F}^{+}(x) H^{+}(x)+\mathcal{F}^{-}(x) H^{-}(x)+o(1), \quad n \rightarrow \infty
\end{aligned}
$$

Отсюда, воспользовавшись представлениями (99) и (100) для граничных значений функций $H$ и $\mathcal{F}$ на отрезке $\Delta$, получаем

$$
\begin{aligned}
Q_{n}(x ; f) & =\frac{1}{\sqrt{\rho(x)}}\left\{e^{n i \varphi(x)+i \alpha(x)+i \theta(x)}+e^{-n i \varphi(x)-i \alpha(x)-i \theta(x)}\right\}+o(1) \\
& =\frac{2}{\sqrt{\rho(x)}} \cos (n \arccos x+\theta(x)+\alpha(x))+o(1),
\end{aligned}
$$

где $o(1)=o\left(\delta^{n}\right), \delta<1$,

$$
\theta(x)=\frac{\sqrt{1-x^{2}}}{2 \pi} \int_{\Delta} \frac{\log \rho(t)-\log \rho(x)}{x-t} \frac{d t}{\sqrt{1-t^{2}}} .
$$

Следствие 3 доказано.

\section{§ 7. Случай нескольких отрезков}

1. В этом параграфе мы коротко изложим результаты, относящиеся к случаю, когда носитель меры $\sigma$ марковской функции (5) состоит из нескольких (непересекающихся) отрезков: $S=$ $\bigsqcup_{j=1}^{p} \Delta_{j} \Subset \mathbb{R}, p \geqslant 2$. В [20] Е. А. Рахманов получил для такого случая аналог теоремы 1 для мероморфных функций вида $f=\widehat{\sigma}+r$, 
где $r$ - вещественная рациональная функция, все полюсы которой лежат вне отрезка $\Delta$ - выпуклой оболочки $S$. В той же работе [20] доказано, что уже для двух отрезков и комплексной рациональной функции $r$ аналог теоремы 1 не имеет места.

ТЕорема 1'. Пусть $\sigma$ - положительная борелевская мера на $\mathbb{R}$, носитель $S=\operatorname{Supp} \sigma$ которой состоит из нескольких отрезков, $\Delta$ - выпуклая оболочка $S$ u $f=\widehat{\sigma}+r$, где $r$ - произвольная вещественная ращиональная функция, все полюсы которой принадлежат области $\mathbb{C} \backslash \Delta$, u $r(\infty)=0$. Тогда последовательность диагональных аппроксимаиий Паде $\left\{f_{n}\right\}_{n \in \mathbb{N}}$ сходится $\kappa f$ равномерно в сферической метрике внутри области $\widetilde{D}=\widehat{\mathbb{C}} \backslash \Delta$.

При этом, как и в теореме 1, к каждому полюсу $f$ в $\widetilde{D}$ стремится ровно столько полюсов $f_{n}$, какова его кратность. Остальные полюсы $f_{n}$ ведут себя следующим образом. Все предельные точки этих полюсов лежат на отрезке $\Delta$; ассоциированная с полюсами $f_{n}$ единичная ${ }^{12}$ мера (слабо) сходится к равновесной мере компакта $S$, в каждой из $p-1$ (открытой) лакуне между отрезками при фиксированном $n \in \mathbb{N}$ может располагаться не больше чем по одному полюсу $f_{n}$, в "общем положении" такие полюсы $f_{n}$ при $n \rightarrow \infty$ всюду плотны в лакунах. Тем самым, в рассматриваемом случае "как правило" весь отрезок $\Delta-$ выпуклая оболочка $S$ - состоит из предельных точек полюсов $f_{n}$.

Используемый в настоящей работе метод исследования сильной асимптотики знаменателей $Q_{n}(z ; f)$ рациональных дробей $f_{n}$ оказывается применим и для рассмотренного Е. А. Рахмановым случая, когда $S=\bigsqcup_{j=1}^{p} \Delta_{j}$ в предположении, что мера $\sigma$ абсолютно непрерывна относительно меры Лебега на $S$ и

$$
\frac{d \sigma}{d x}=\frac{1}{\pi} \frac{\rho(x)}{\sqrt{-h(x+i 0)}}>0 \quad \text { на } S \text {; }
$$

здесь $h(z)=\prod_{j=1}^{2 p}\left(x-e_{j}\right), e_{1}, \ldots, e_{2 p}$ - концы отрезков $\left\{\Delta_{j}\right\}$, в области $D=\widehat{\mathbb{C}} \backslash S$ выбрана ветвь квадратного корня, которая положительна при положительных значениях аргумента, а весовая

\footnotetext{
${ }^{12} \mathrm{~B}$ условиях теоремы $1^{\prime}$ все индексы $n$ нормальны, тем самым, при каждом $n$ рациональная функция $f_{n}$ имеет ровно $n$ полюсов.
} 
функция $\rho$ голоморфна и не обращается в нуль на $S$. Формулы сильной асимптотики для $Q_{n}$ и в этом случае выражаются явно в терминах $\Psi$-функции - решения соответствующей специальной краевой задачи Римана - и вполне аналогичны $\left(22_{1}\right)-\left(22_{3}\right)$. Однако при этом в дивизоре $\Psi$-функции неизбежно присутствуют "свободные" нули, число которых равно числу лакун между отрезками, а их поведение при $n \rightarrow \infty$ определяется проблемой обращения Якоби.

2. Пусть $\mathfrak{R}$ - гиперэллиптическая риманова поверхность рода $g=p-1$, заданная уравнением $w^{2}=h(z)$, где $h(z)=\prod_{j=1}^{2 g+2}\left(z-e_{j}\right)$, $e_{1}<\cdots<e_{2 g+2}$. Будем считать, что $\mathfrak{R}$ реализована как двулистное разветвленное в точках $\left\{e_{j}\right\}$ накрытие римановой сферы $\widehat{\mathbb{C}}$ таким образом, что переход с одного листа на другой осуществляется по верхнему $\Delta_{j}^{+}$и нижнему $\Delta_{j}^{-}$берегам отрезков $\Delta_{j}$. Тем самым, над каждой точкой $\widehat{\mathbb{C}}$, за исключением точек ветвления $\left\{e_{j}\right\}$, лежат ровно две точки римановой поверхности, а каждому отрезку $\Delta_{j}$ соответствует на $\mathfrak{R}$ замкнутая аналитическая (в комплексной структуре $\mathfrak{R}$ ) кривая $\Gamma_{j}, j=1, \ldots, g+1$, - цикл на $\mathfrak{R}$; положим $\Gamma=\bigcup_{j=1}^{g+1} \Gamma_{j}$. Будем считать, что в $D=$ $\widehat{\mathbb{C}} \backslash S$ выбрана ветвь квадратного корня, удовлетворяющая условию $\sqrt{h(z)} / z^{g+1} \rightarrow 1$ при $z \rightarrow \infty$. Функция $w: w^{2}=h(z)$ однозначна на $\mathfrak{R}$. Первым (открытым) листом $D^{(1)}$ поверхности $\mathfrak{R}$ будем считать тот, на котором $w=\sqrt{h(z)}$. На втором листе $D^{(2)}$ имеем $w=-\sqrt{h(z)}$. Для точек римановой поверхности $\mathfrak{R}$ будем использовать обозначение $\mathbf{z}=(z, w)$, где $w= \pm \sqrt{h(z)}$; при этом для точек первого листа $z^{(1)}=(z, \sqrt{h(z)})$, а для точек второго $z^{(2)}=(z,-\sqrt{h(z)})$. Область $D^{(1)}$ будем, как правило, отождествлять с "физической" областью $D$. Для $\mathbf{z}=z^{(1)}$ будем часто писать просто $w(z)$ вместо $w(\mathbf{z})$; тем самым, приобретает смысл и запись $w^{+}(x)=\sqrt{h(x+i 0)}, x \in S$ (см. (101)). Каноническая проекция pr: $\mathfrak{R} \rightarrow \widehat{\mathbb{C}}$ определяется соотношением pr $\mathbf{z}=z$, в частности $\operatorname{pr} D^{(1)}=\operatorname{pr} D^{(2)}=D, \operatorname{pr} \Gamma=S$.

Приведем некоторые стандартные сведения о гиперэллиптических римановых поверхностях, которые понадобятся нам в этом параграфе. В обозначениях и терминологии мы здесь придерживаемся в основном [19] (см. также [21], [22]). 
Ориентируем кривые $\Gamma_{j}$ так, что при обходе по $\Gamma_{j}$ область $D^{(1)}$ остается слева, а область $D^{(2)}$ - справа. Примем ориентированные кривые $\Gamma_{j}, j=1, \ldots, g$, за $а$-циклы $\mathbf{a}_{j}$ на $\mathfrak{R}$ и стандартным образом [19] дополним их $b$-циклами $\mathbf{b}_{j}$ до базиса гомологий $\left\{\mathbf{a}_{j}, \mathbf{b}_{j}\right\}_{j=1, \ldots, g}$ на $\mathfrak{R}$. Пусть $\left\{d \Omega_{k}\right\}$ - соответствующий $a$-нормированный базис голоморфных абелевых дифференциалов:

$$
\oint_{\mathbf{a}_{j}} d \Omega_{k}=\delta_{k j}, \quad k, j=1, \ldots, g .
$$

Матрица $\mathbf{B}=\left\|B_{k j}\right\|_{k, j=1, \ldots, g}$, где $B_{k j}=\oint_{\mathbf{b}_{j}} d \Omega_{k}-b$-периоды базисных дифференциалов, является матрицей Римана: она симметрична, $B_{k j}=B_{j k}$, а ее мнимая часть положительно определена, $\left\|\operatorname{Im} B_{j k}\right\|>0$ (отметим, что в рассматриваемом здесь случае все $\left.B_{j k} \in i \mathbb{R}\right)$. Функции

$$
\Omega_{k}(\mathbf{z})=\int_{e_{2 g+1}}^{\mathbf{z}} d \Omega_{k}
$$

определены на $\mathfrak{R}$ однозначно по модулю их $a$ - и $b$-периодов. Так как $\Omega_{k}^{\prime}(z)=p_{k}(z) / w(z)$, где $p_{k}-$ полином степени $g-1$, то имеет смысл обозначение $d \Omega_{k}^{+}(x)=p_{k}(x) d x / w^{+}(x)$ при $x \in S$.

Если в $\mathfrak{R} \backslash \Gamma$ задана функция $F(\mathbf{z})$, то под $F(\mathbf{x}+)$ понимаются ее предельные значения при $\mathbf{z} \rightarrow \mathbf{x} \in \Gamma, \mathbf{z} \in D^{(1)}$, если они существуют; аналогичный смысл придается и $F(\mathbf{x}-)$ при $\mathbf{z} \in D^{(2)}$, $\mathbf{z} \rightarrow \mathbf{x} \in \Gamma$.

Фиксируем стандартный базис $\mathbf{e}_{1}, \mathbf{e}_{2}, \ldots, \mathbf{e}_{g}$ в $\mathbb{R}^{g}:\left(\mathbf{e}_{k}\right)_{j}=\delta_{k j}$. Тогда векторы $\mathbf{e}_{1}, \ldots, \mathbf{e}_{g}, \mathbf{B e}_{1}, \ldots, \mathbf{B e}_{g}$ линейно независимы над $\mathbb{R}$ и образуют базис в $\mathbb{C}^{g}$. Факторпространство $\mathbb{C}^{g} /\{\mathrm{N}+\mathbf{B M}\}$ по целочисленной решетке, $\mathrm{N}, \mathrm{M} \in \mathbb{Z}^{g}$, является $2 g$-мерным вещественным тором $\mathbb{T}^{2 g}$ и называется многообразием Якоби Јас $\Re$ римановой поверхности $\mathfrak{R}$. Любой вектор $\mathbf{v} \in \mathbb{C}^{g}$ однозначно представим в виде $\mathbf{v}=\mathbf{x}+\mathbf{B y}+\mathbf{N}+\mathbf{B M}=\mathbf{x}+\mathbf{B y}(\bmod$ периодов $)$, $0 \leqslant(\mathbf{x})_{j},(\mathbf{y})_{j}<1, \mathrm{~N}, \mathrm{M} \in \mathbb{Z}^{g}$; иногда для краткости знак сравнения по модулю периодов дифференциалов $\left\{d \Omega_{k}\right\}$ мы будем опускать и писать просто $\mathbf{v} \equiv \mathbf{x}+\mathbf{B y}$.

Неупорядоченные наборы точек $\left(\mathbf{z}_{1}, \ldots, \mathbf{z}_{g}\right), \mathbf{z}_{j} \in \mathfrak{R}$, образуют $g$-ю симметрическую степень $S^{g} \mathfrak{R}$ римановой поверхности $\mathfrak{R}$. 
Пусть $\mathbf{z}_{0}$ - некоторая фиксированная точка ${ }^{13} \mathfrak{R}$. Вектор-функция $A\left(\mathbf{z}_{1}, \ldots, \mathbf{z}_{g}\right)=\left(A_{1}, \ldots, A_{g}\right)$ с координатами

$$
A_{k}=A_{k}\left(\mathbf{z}_{1}, \ldots, \mathbf{z}_{g}\right) \equiv \sum_{j=1}^{g} \Omega_{k}\left(\mathbf{z}_{j}\right) \equiv \sum_{j=1}^{g} \int_{\mathbf{z}_{0}}^{\mathbf{z}_{j}} d \Omega_{k}, \quad k=1, \ldots, g
$$

определяет отображение Абеля $A: S^{g} \mathfrak{R} \rightarrow$ Јас $\mathfrak{R}$. Если задан вектор $\mathbf{v}=\left(v_{1}, \ldots, v_{g}\right) \in \mathrm{Jac} \mathfrak{R}$, то задача нахождения неупорядоченного набора точек $\left(\mathbf{z}_{1}, \ldots, \mathbf{z}_{g}\right) \in S^{g} \Re$, для которого

$$
A_{k}\left(\mathbf{z}_{1}, \ldots, \mathbf{z}_{g}\right)=v_{k} \quad(\bmod \text { периодов }), \quad k=1, \ldots, g,
$$

называется проблемой обращения Якоби. Проблема обращения Якоби всегда разрешима, но, вообще говоря, не единственным образом. В дальнейшем неупорядоченный набор точек $\left(\mathbf{z}_{1}, \ldots, \mathbf{z}_{g}\right) \in$ $S^{g} \mathfrak{R}$ будем называть дивизором и обозначать $d=\mathbf{z}_{1}+\cdots+\mathbf{z}_{g}$.

Для любых двух различных точек $\mathbf{z}_{1}$ и $\mathbf{z}_{2}$ римановой поверхности $\mathfrak{R}$ существует абелев дифференциал третьего рода, голоморфный всюду на $\mathfrak{R}$, за исключением этих двух точек, в которых он имеет простые полюсы с вычетами соответственно 1 и -1 ; такой дифференциал называется нормальным. Нормальный дифференциал будет определен однозначно, если потребовать, чтобы все его $a$-периоды были равны нулю; будем называть такой дифференциал $a$-нормированным абелевым дифференциалом третьего рода и обозначать $d \Omega\left(\mathbf{z}_{1}, \mathbf{z}_{2} ; \mathbf{z}\right)$. Другой способ однозначно задать (нормировать) дифференциал третьего рода - потребовать, чтобы все его периоды были чисто мнимыми. Для таких дифференциалов будем использовать обозначение $d G\left(\mathbf{z}_{1}, \mathbf{z}_{2} ; \mathbf{z}\right)$, предполагая при этом, что вычеты в точках $\mathbf{z}_{1}$ и $\mathbf{z}_{2}$ равны соответственно -1 и $1 . \mathrm{K}$ подобным дифференциалам относится дифференциал комплексной функции Грина $G_{D}(z, \infty)=g_{D}(z, \infty)+i g_{D}^{*}(z, \infty)$ (здесь $g_{D}^{*}(z, \infty)$ - функция, гармонически сопряженная к функции Грина $\left.g_{D}(z, \infty)\right)$ многосвязной области $D=\widehat{\mathbb{C}} \backslash S$. Функция $G_{D}(z, \infty)$ многозначна в $D$ и

$$
G_{D}(z, \infty)=\int_{e_{2 g+1}}^{z} \frac{P_{g}(\zeta)}{\sqrt{h(\zeta)}} d \zeta, \quad z \in D
$$

\footnotetext{
${ }^{13} \mathrm{~B}$ дальнейшем мы выбираем $\mathbf{z}_{0}=e_{2 g+1}$.
} 
где $P_{g}(z)=P_{g}(z ; h)=z^{g}+\cdots-$ вещественный полином, все нули которого лежат в лакунах $\left(e_{2 j}, e_{2 j+1}\right), j=1, \ldots, g$. Дифференциал $d G=d G\left(\infty^{(1)}, \infty^{(2)} ; \mathbf{z}\right)=P_{g}(z) d z / w(\mathbf{z})$ определен на всей римановой поверхности $\mathfrak{R}$, имеет чисто мнимые периоды и простые полюсы в точках $\mathbf{z}=\infty^{(1)}$ и $\mathbf{z}=\infty^{(2)}$ с вычетами соответственно -1 и 1.

Через $\omega_{k}(z ; D)=\omega\left(z ; \Delta_{k}, D\right)$ будем обозначать гармоническую меру (в точке $z \in D$ ) отрезка $\Delta_{k}$ относительно области $D$.

3. Следуя предложенной в $\S 2$ схеме изложения, обсудим сначала случай $f=\widehat{\rho}$, где

$$
\widehat{\rho}(z)=\frac{1}{\pi i} \int_{S} \frac{\rho(x)}{z-x} \frac{d x}{w^{+}(x)},
$$

компакт $S$ состоит из нескольких отрезков, а весовая функция $\rho$ голоморфна на $S$ и удовлетворяет условию (101).

Зафиксируем произвольное $n \in \mathbb{N}$ и рассмотрим на римановой поверхности $\mathfrak{R}$ следующую краевую задачу Римана.

ЗАДАЧА $\left(\mathcal{R}_{1}^{\prime}\right)$. При фиксированном $n \in \mathbb{N}, n \geqslant g$, найти функиию $\Psi=\Psi_{n}$ такую, что:

$1^{\circ} . \Psi$ (кусочно) мероморфна на $\Re \backslash \Gamma=D^{(1)} \sqcup D^{(2)}$;

$2^{\circ}$. дивизор $(\Psi)=(n-g) \infty^{(2)}+\mathbf{z}_{1}+\cdots+\mathbf{z}_{g}-n \infty^{(1)}$;

$3^{\circ}$. на Г выполнено краевое условие: $\rho(x) \Psi(\mathbf{x}+)=\Psi(\mathbf{x}-)$.

В п. $2^{\circ}$ точки $\mathbf{z}_{j}-$ "свободные" нули $\Psi$-функции - зависят от $n$, под $\Psi(\mathrm{x}+)$ в п. $3^{\circ}$ понимаются предельные значения функции $\Psi(\mathbf{z})$ при $D^{(1)} \ni \mathbf{z} \rightarrow \mathbf{x} \in \Gamma$, аналогичный смысл придается и $\Psi(\mathbf{x}-)$. Функция $\Psi$, решающая задачу $\left(\mathcal{R}_{1}^{\prime}\right)$, всегда существует, при этом так как род $g=p-1$ поверхности $\mathfrak{R}$ положителен, то нули и полюсы $\Psi$ на $\mathfrak{R}$ оказываются связанными определенными соотношениями, аналогичными соотношениям Абеля для произвольной мероморфной функции на $\mathfrak{R}$, а дивизор $d=\mathbf{z}_{1}+\cdots+\mathbf{z}_{g}$ является решением определенной проблемы обращения Якоби. Анализ данных этой проблемы показывает, что при оговоренном выше условии (101) на вес $\rho$ ее решение всегда таково, что $z_{j}=\operatorname{pr} \mathbf{z}_{j} \in\left[e_{2 j}, e_{2 j+1}\right]$, т.е. в каждой замкнутой лакуне между отрезками $\left\{\Delta_{j}\right\}$ лежит ровно по одной точке $\operatorname{pr} \mathbf{z}_{j} \in \mathbb{R}$. Значит, дивизор $d=\mathbf{z}_{1}+\cdots+\mathbf{z}_{g}$ неспециальный, 
а следовательно, такая проблема обращения Якоби имеет единственное решение. Отсюда вытекает, что $\Psi$-функция, решающая задачу $\left(\mathcal{R}_{1}^{\prime}\right)$, единственна с точностью до нормировки и имеет в бесконечно удаленной точке $\mathbf{z}=\infty^{(1)}$ полюс в точности $n$-го порядка. Нетрудно видеть, что для любого $z \in \mathbb{C} \backslash S$ выполняется соотношение $\Psi\left(z^{(1)}\right) \Psi\left(z^{(2)}\right) \equiv$ const $\cdot \prod_{j=1}^{g}\left(z-z_{j}\right)$, где const $\neq 0$. В дальнейшем мы будем придерживаться следующей нормировки ${ }^{14} \Psi$-функции: $\Psi\left(z^{(1)}\right) \Psi\left(z^{(2)}\right) \equiv \prod_{j=1}^{g}\left(z-z_{j}\right)$. Нетрудно найти и явный вид этой функции. При $\mathbf{z}=z^{(1)} \in D^{(1)}$ и в предположении, что $z_{j} \in\left(e_{2 j}, e_{2 j+1}\right)$, имеем (ср. с $\left.(21)\right)$ :

$$
\Psi\left(z^{(1)}\right)=\Phi(z)^{n-g} e^{A(z ; \rho)} \mathcal{F}_{n}(z),
$$

где $\Phi(z)=e^{G_{D}(z, \infty)}-($ многозначная) отображающая функция,

$$
\begin{gathered}
A(z ; \rho)=w(z)\left(\frac{1}{2 \pi i} \int_{S} \frac{\log \rho(x)}{x-z} \frac{d x}{w^{+}(x)}\right. \\
\left.+\frac{1}{2 \pi i} \sum_{k=1}^{g} \lambda_{k} \int_{\Delta_{k}} \frac{1}{x-z} \frac{d x}{w^{+}(x)}\right), \\
\lambda_{k}=2 \int_{S} \log \rho(x) d \Omega_{k}^{+}(x)(\text { при } g=0 \quad A(z ; \rho)-\text { функция Сегё }), \\
\mathcal{F}_{n}(z)=\exp \left(\sum_{j=1}^{g} \Omega\left(\mathbf{z}_{j}, \infty{ }^{(1)} ; z^{(1)}\right)+\frac{1}{2} c_{g}+\sum_{k=1}^{g} \theta_{k} \Omega_{k}\left(z^{(1)}\right)\right) \\
=\exp \left(\varphi_{n}(z)\right),
\end{gathered}
$$

величины $\theta_{k}=\theta_{k}(n)=\left\{n \omega_{k}(\infty)\right\}-g$ (здесь и ниже в (105) $\{\cdot\}$ обозначает дробную часть соответствующего числа), дивизор $d=$ $\mathbf{z}_{1}+\cdots+\mathbf{z}_{g}$, где $\mathbf{z}_{j}=\mathbf{z}_{j}(n)$, является (единственным) решением проблемы обращения Якоби

$$
\begin{gathered}
\sum_{j=1}^{g} \Omega_{k}\left(\mathbf{z}_{j}\right)=\sum_{s=1}^{g}\left\{\left(n-\frac{1}{2}\right) \omega_{s}(\infty ; D)\right\} B_{k s}+\frac{i}{\pi} \int_{S} \log \rho(x) d \Omega_{k}^{+}(x) \\
k=1, \ldots, g
\end{gathered}
$$

величина $c_{g}=c_{g}(n)=\prod_{j=1}^{g}\left(e_{2 g+1}-z_{j}\right)$.

\footnotetext{
${ }^{14}$ Как уже отмечалось выше, таким условием $\Psi$-функция определяется однозначно с точностью до знака \pm .
} 
Представление для $\Psi\left(z^{(2)}\right)$ при $z^{(2)} \in D^{(2)}$ следует непосредственно из (103) и соотношения $\Psi\left(z^{(1)}\right) \Psi\left(z^{(2)}\right) \equiv \prod_{j=1}^{g}\left(z-z_{j}\right)$ при $z \in D$. Так как функция $\rho$ голоморфна и отлична от нуля на $S$, то правая часть представления (103) имеет смысл и при $\mathbf{z} \in D^{(1)} \sqcup \Gamma$ для дивизора $d=\mathbf{z}_{1}+\cdots+\mathbf{z}_{g}$, удовлетворяющего системе уравнений (105). Тем самым, под функцией $\Psi(\mathbf{z}), \mathbf{z} \in D^{(1)} \sqcup \Gamma$, естественно понимать правую часть представления (105). Аналогичного соглашения будем придерживаться и для функции $\Psi(\mathbf{z})$ при $\mathbf{z} \in D^{(2)} \sqcup \Gamma$.

При подходящей нормировке полиномов $Q_{n}(z ; \widehat{\rho})$ - знаменателей диагональных аппроксимаций Паде функции $f=\widehat{\rho}-$ их сильная асимптотика внутри области $\widetilde{D}=\widehat{\mathbb{C}} \backslash \Delta$ и на компакте $S$ описывается в терминах $\Psi$-функции, решающей задачу $\left(\mathcal{R}_{1}^{\prime}\right)$, следующим образом.

ПРЕДЛОЖЕНИЕ $1^{\prime}$. Пусть голоморфная на $S$ функиия $\rho$ удовлетворяет условию (101), $\Psi$ - решение задачи $\left(\mathcal{R}_{1}^{\prime}\right)$ при $n \in \mathbb{N}$. Тогда при подходящей нормировке полиномов $Q_{n}(z ; \widehat{\rho})$ имеем:

$$
\begin{aligned}
1^{\circ} . & Q_{n}(z ; \widehat{\rho})=\Psi(z)(1+o(1)) \text { при } n \rightarrow \infty \text { равномерно внутри } \\
& \widetilde{D}=\widehat{\mathbb{C}} \backslash \Delta ; \\
2^{\circ} . & Q_{n}(x ; \widehat{\rho})=\Psi^{+}(x)+\Psi^{-}(x)+o(1) \text { при } n \rightarrow \infty \text { равномерно } \\
& \text { на } S .
\end{aligned}
$$

Здесь $\Psi(z)=\Psi\left(z^{(1)}\right)$, под $\Psi^{+}(x)\left(\Psi^{-}(x)\right)$ понимаются верхние (соответственно нижние) предельные значения $\Psi(z)$ на $S$; величина $o(1)=o\left(\delta^{n}\right)$, где $\delta<1$. Функция $\Psi$, решающая задачу $\left(\mathcal{R}_{1}^{\prime}\right)$, имеет в точке $\mathbf{z}=\infty^{(1)}$ полюс в точности $n$-го порядка. Так как $\operatorname{deg} Q_{n}=n$ при всех $n$ и $\Psi\left(z^{(1)}\right) / z^{n} \rightarrow \varkappa_{n} \neq 0$ при $z \rightarrow \infty$, где $\varkappa_{n} \in \mathbb{R}$, то естественно нормировать $Q_{n}$ условием $Q_{n}(z)=\varkappa_{n} z^{n}+\cdots$. С помощью представления (103) величину $\varkappa_{n}$ нетрудно найти и в явном виде, при этом, так как $\varkappa_{n} \in \mathbb{R}$, можно считать, что $\varkappa_{n}>0$; таким выбором знака у $\varkappa_{n}$ однозначно определяется и сама $\Psi$-функция.

Отметим, что сильная асимптотика полиномов $Q_{n}$ в лакунах между отрезками нам здесь не понадобится, хотя она также может быть описана в терминах, связанных с римановой поверхностью $\mathfrak{R}$ (так как асимптотическое поведение нулей $Q_{n}$, расположенных в лакунах между отрезками, подчиняется определенной динамической системе; см. [17] и [20]). 
4. Перейдем теперь к общему случаю $f=\widehat{\rho}+r$, где функция $\rho$ удовлетворяет условию (101) и голоморфна на $S$, а функция $r \in$ $\mathbb{R}(z)$ и не имеет полюсов на $\Delta-$ выпуклой оболочке $S$. Пусть $a_{1}, \ldots, a_{\ell}$ - полюсы $f$ в $\mathbb{C} \backslash \Delta, m_{1}, \ldots, m_{\ell}-$ их кратности.

Рассмотрим на римановой поверхности $\mathfrak{R}$ следующую краевую задачу Римана.

ЗАДАчА $\left(\mathcal{R}_{2}^{\prime}\right)$. При фиксированном $n \in \mathbb{N}, n \geqslant g$, найти функцию $\Psi=\Psi_{n}$ такую, что:

$1^{\circ}$. $\Psi$ (кусочно) мероморфна на $\mathfrak{R} \backslash \Gamma=D^{(1)} \sqcup D^{(2)}$;

$2^{\circ}$. дивизор $(\Psi)=(n-g) \infty^{(2)}+\mathbf{z}_{1}+\cdots+\mathbf{z}_{g}+m_{1} a_{1}^{(1)}+\cdots+$ $m_{\ell} a_{\ell}^{(1)}-m_{1} a_{1}^{(2)}-\cdots-m_{\ell} a_{\ell}^{(2)}-n \infty^{(1)}$;

$3^{\circ}$. на Г выполнено краевое условие: $\rho(x) \Psi(\mathbf{x}+)=\Psi(\mathbf{x}-)$.

Как и в задаче $\left(\mathcal{R}_{1}^{\prime}\right)$, точки $\mathbf{z}_{j}$ в п. $2^{\circ}$ зависят от $n$. Смысл обозначений $\Psi(\mathrm{x}+)$ и $\Psi(\mathrm{x}-)$ - тот же, что и выше. При оговоренных выше условиях на $\rho$ и $r$ функция $\Psi$, решающая задачу $\left(\mathcal{R}_{2}^{\prime}\right)$, всегда существует, при этом дивизор $d=\mathbf{z}_{1}+\cdots+\mathbf{z}_{g}$ оказывается единственным решением некоторой проблемы обращения Якоби, кроме того, $z_{j} \in\left[e_{2 j}, e_{2 j+1}\right]$ при $j=1, \ldots, g$, т.е. в каждой замкнутой лакуне между $\left\{\Delta_{j}\right\}$ лежит ровно по одной точке $z_{j}=\operatorname{pr} \mathbf{z}_{j}$. Функция, решающая задачу $\left(\mathcal{R}_{2}^{\prime}\right)$, единственна с точностью до нормировки и имеет в бесконечно удаленной точке $\mathbf{z}=\infty^{(1)}$ полюс в точности $n$-го порядка. Для любого $z \in \mathbb{C} \backslash S$ выполняется соотношение $\Psi\left(z^{(1)}\right) \Psi\left(z^{(2)}\right) \equiv$ const $\cdot \prod_{j=1}^{g}\left(z-z_{j}\right)$, где const $\neq 0$. Как и ранее, мы придерживаемся следующей нормировки $\Psi$-функции: $\Psi\left(z^{(1)}\right) \Psi\left(z^{(2)}\right) \equiv \prod_{j=1}^{g}\left(z-z_{j}\right)$. Если условиться, что $\Psi\left(z^{(1)}\right) / z^{n} \rightarrow \varkappa_{n}>0$ при $z \rightarrow \infty$, то последним тождеством $\Psi$-функция определяется однозначно. По сравнению с задачей $\left(\mathcal{R}_{1}^{\prime}\right)$ явный вид этой функции претерпевает следующие изменения: в правую часть (103) надо добавить сомножитель

$$
\Pi_{m}(z)=\exp \left\{\sum_{s=1}^{\ell} m_{s} \Omega\left(a_{s}^{(1)}, a_{s}^{(2)} ; z^{(1)}\right)\right\}
$$

(здесь $m=\sum_{s=1}^{\ell} m_{s}$ - полное число полюсов $f$ в $\mathbb{C} \backslash \Delta$ ), а в правую часть $(105)$ - удвоенное слагаемое $\sum_{s=1}^{\ell} m_{s} \Omega_{k}\left(a_{s}^{(1)}\right)$. В итоге получаем, что при $z \in \mathbb{C} \backslash S$

$$
\Psi\left(z^{(1)}\right)=\Phi(z)^{n-g} e^{A(z ; \rho)} \mathcal{F}_{n}(z) \Pi_{m}(z),
$$


где дивизор $\mathbf{z}_{1}+\cdots+\mathbf{z}_{g}$ является (единственным) решением следующей проблемы обращения Якоби:

$$
\begin{aligned}
\sum_{j=1}^{g} \Omega_{k}\left(\mathbf{z}_{j}\right)=\sum_{s=1}^{g}\left\{\left(n-\frac{1}{2}\right) \omega_{s}(\infty ; D)\right\} B_{k s}+\frac{i}{\pi} \int_{S} \log \rho(x) d \Omega_{k}^{+}(x) \\
+2 \sum_{s=1}^{\ell} m_{s} \Omega_{k}\left(a_{s}^{(1)}\right), \quad k=1, \ldots, g .
\end{aligned}
$$

В терминах $\Psi$-функции, решающей задачу $\left(\mathcal{R}_{2}^{\prime}\right)$, асимптотическое поведение полиномов $Q_{n}(z ; f)$, нормированных условием $Q_{n}(z ; f)=\varkappa_{n} z^{n}+\cdots$, описывается так.

ПРЕДЛОЖЕНИЕ $2^{\prime}$. Пусть $f=\widehat{\rho}+r$, где голоморфная на $S$ функиия $\rho$ удовлетворяет условию (101), вещественная рациональная функиия $r$ не имеет полюсов на $\Delta$ u $r(\infty)=0$. Если $\Psi$ - решение задачи $\left(\mathcal{R}_{2}\right)$ при $n \in \mathbb{N}$, то для полиномов $Q_{n}(z ; f)$ имеем:

$1^{\circ} . Q_{n}(z ; f)=\Psi(z)(1+o(1))$ при $n \rightarrow \infty$ равномерно внутри $\widetilde{D}^{\prime}$,

$2^{\circ} . Q_{n}(x ; f)=\Psi^{+}(x)+\Psi^{-}(x)+o(1)$ при $n \rightarrow \infty$ равномерно на $S$,

где $\widetilde{D}=\widehat{\mathbb{C}} \backslash \Delta, \quad \widetilde{D}^{\prime}=\widetilde{D} \backslash\{$ полюсъ функиии $f\}$, величина о $(1)=$ $o\left(\delta^{n}\right), \delta<1$.

Формула п. $2^{\circ}$ сильной асимптотики для полиномов $Q_{n}(x ; f)$ на $S$ лежит в основе доказательства следующего утверждения об асимптотическом поведении полюсов $f_{n}$ в окрестности кратного полюса $f$.

Теорема $3^{\prime}$. Пусть в условиях предложения $2^{\prime}$ точка $a_{j} \in D$ - полюс функиии $f$ кратности $m_{j} \geqslant 1, a_{j, \nu}(n), \nu=1, \ldots, m_{j}$, соответствующие точке $a_{j}$ при $n \rightarrow \infty$ полюсы $f_{n}$. Тогда

$$
a_{j, \nu}(n)=a_{j}+\frac{C_{j} \varepsilon_{j}^{\nu}}{B_{j}^{2 n}}\left(1+o\left(\delta^{n}\right)\right) \quad n p u \quad n \rightarrow \infty, \quad \nu=1, \ldots, m_{j},
$$




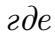

$$
\begin{gathered}
\varepsilon_{j}=e^{2 \pi i / m_{j}}, \\
B_{j}=\Phi\left(a_{j}\right)^{1 / m_{j}}=e^{G\left(a_{j}\right) / m_{j}}, \quad\left|B_{j}\right|>1 \\
C_{j}=C_{j}(n)=e^{-2 A\left(a_{j} ; \rho\right) / m_{j}}\left(-\frac{2 G^{\prime}\left(a_{j}\right) r_{n, g}\left(a_{j}\right)}{A_{j} \alpha_{j}}\right)^{2 / m_{j}} \\
\quad \times e^{2 g G\left(a_{j}\right) / m_{j}-2 \varphi_{n}\left(a_{j}\right) / m_{j}}\left(\frac{m_{j} !}{\Pi_{m}^{\left(m_{j}\right)}\left(a_{j}\right)}\right)^{2 / m_{j}}
\end{gathered}
$$

Здесь функция $r_{n, g}(z)=P_{g}(z) / \prod_{j=1}^{g}\left(z-z_{j}\right)$ и, тем самым, зависит от $n$, комплексные постоянные $A_{j}=\left(f \omega_{m}\right)\left(a_{j}\right) \neq 0, \alpha_{j}=$ $\omega_{m}^{\left(m_{j}\right)}\left(a_{j}\right) / m_{j} ! \neq 0, j=1, \ldots, \ell$, зависят только от $f$, функция $\varphi_{n}$ определена соотношением (104).

\section{Список литературы}

[1] Бейкер Дж., Грейвс-Моррис П., Аппроксимации Паде. - М.: Мир, 1986.

[2] Чебышёв П. Л., "О непрерывных дробях" // Ученые записки Имn. Академии Наук, 1855, III, 636-664. (Полное собрание сочинений. Т. II. М.-Л.: Изд-во АН СССР, 1948, 103-126.)

[3] Марков A.A., "Deux démonstrations de la convergence de certaines fractions continues" // Acta Math., 1895, 19, 93104. (Два доказательства сходимости некоторых непрерывных дробей / / Избранные труды по теории непрерывных дробей и теории функций, наименее уклоняющихся от нуля. М.: Гостехиздат, 1948, 106-119.)

[4] Гончар А. А., "О сходимости аппроксимаций Паде для некоторых классов мероморфных функций" // Mamем. сб., 1975, 97 (139), 607-629.

[5] Рахманов Е. А., "Об асимптотике отношения ортогональных многочленов" // Матем. сб., 1977, 103 (145), 237-252.

[6] Рахманов Е. А., "Об асимптотике отношения ортогональных многочленов. II" // Mameм. сб., 1982, 118 (160), 104-117.

[7] Гончар А. А., "О равномерной сходимости диагональных аппроксимаций Паде" // Матем. сб., 1982, 118 (160), 535-556. 
[8] Гончар А.А., "О сходимости диагональных аппроксимаций Паде в сферической метрике" // Математические структуры - вычислительная математика - математическое моделирование. Т. 2. София, 1984, 29-35.

[9] Бернштейн С. Н., О многочленах, ортогоналъных в конечном интервале. - Харьков: ОНТИ, 1937.

[10] Ахиезер Н. И., "Об ортогональных многочленах на нескольких интервалах" // Докл. АН СССР, 1960, 134(1), 9-12.

[11] Ахиезер Н. И., Томчук Ю. Я., "К теории ортогональных многочленов на нескольких интервалах" // Докл. АН СССР, 1961, 138(4), 743-746.

[12] Ахиезер Н. И., "Континуальные аналоги ортогональных многочленов на системе интервалов" // Докл. АН СССР, 1961, 141(2), 263-266.

[13] Nuttall J., "Asymptotics of diagonal Hermite-Padé polynomials" // J. Approx. Theory, 1984, 42, 299-386.

[14] Nuttall J., "Padé polynomial asymptotics from a singular integral equation" // Constr. Approx., 1990, 6(2), 157-166.

[15] Суетин С. П., "О равномерной сходимости диагональных аппроксимаций Паде для гиперэллиптических функций" // Матем. сб., 2000, 191(9), 81-114.

[16] Суетин С.П., "Аппроксимации Паде и эффективное аналитическое продолжение степенного ряда" // УМH, 2002, $\mathbf{5 7}(1), 45-142$.

[17] Суетин С. П., "Об асимптотических свойствах полюсов диагональных аппроксимаций Паде для некоторых обобщений марковских функций" // Матем. сб., 2002, 193(12), 81-114.

[18] Аптекарев А.И., "Точные константы рациональных аппроксимаций аналитических функций" // Матем. сб., 2002, 193(1), 3-72.

[19] Зверович Э.И., "Краевые задачи теории аналитических функций в гёльдеровских классах на римановых поверхностях" // УМН, 1971, 26(1), 113-180.

[20] Рахманов Е. А., "О сходимости диагональных аппроксимаций Паде" // Матем. сб., 1977, 104 (146), 271-291.

[21] Форстер О., Римановы поверхности. - М.: Мир, 1980.

[22] Дубровин Б. А., "Тэта-функции и нелинейные уравнения" // УМH, 1981, 36(2), 11-80. 


\section{Содержание}

$\S 1$. Введение . . . . . . . . . . . . . . . . . . . . . . . 3

$\S 2$. Формулировка основных результатов . . . . . . . . 15

§ 3. Доказательство предложения 1 . . . . . . . . . . . . . 24

$\S 4$. Доказательство теоремы 2 . . . . . . . . . . . . . 28

$\S 5$. Доказательство предложения 2 . . . . . . . . . . . . . . . . 40

§6. Доказательство теоремы 3 . . . . . . . . . . . . . . . . . . 48

$\S 7$. Случай нескольких отрезков . . . . . . . . . . . . 55

Список литературы . . . . . . . . . . . . . . . . . . . . . 65 